\title{
New relationships between spherical and spheroidal harmonics and applications
}

\author{
Matt Majic \\ supervisor: Eric C. Le Ru \\ A thesis \\ submitted to the Victoria University of Wellington \\ in fulfilment of the requirements for the degree of Master of \\ Science
}

Victoria University of Wellington

2016 


\begin{abstract}
This thesis deals with solutions to Laplace's equation in 3D, finding new relationships between solutions, manipulating these to find new approaches to physical problems, and proposing a new class of solutions. We mainly consider spherical and prolate spheroidal geometry and their corresponding solutions - spherical and spheroidal solid harmonics. We first present new relationships between these, expressing for example spherical harmonics as a series of spheroidal harmonics. Similar relationships are known but we work with the spherical and spheroidal coordinate systems being offset from each other. We also propose a new class of solutions which we call logopoles which have many links with spherical and spheroidal harmonics, and are related to the potential created by simple finite line charge distributions. Through the logopoles we find another relationship between the spheroidal harmonics and the often discarded alternate spherical harmonics. Then we apply one of the new spherical-spheroidal harmonic relationships to problems involving a point charge/dipole outside a dielectric sphere. We find new solutions where the potential is expanded as a series of spheroidal harmonics instead of the standard spherical ones, and we show that the convergence is much faster. We also solve these problems with logopoles and the solutions converge even faster, although they are more complicated as they involve a combination of logopoles and spherical harmonics.
\end{abstract}




\section{Acknowledgements}

I would like to thank Eric for making and funding this unique project and being an awesome supervisor, always having ideas and valuable discussions, and giving plenty of fast feedback. Stack exchange was a great resource for external help. And cheers to everyone in the Raman lab for making a great place to work in. 


\section{Contents}

\begin{tabular}{lll}
\hline & Introduction & 1 \\
\hline
\end{tabular}

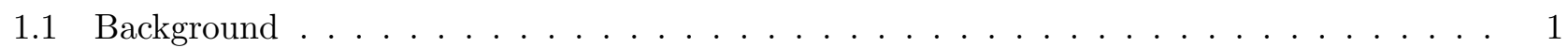

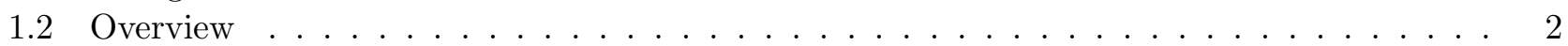

$1.2 .1 \quad$ Spherical and spheroidal harmonics . . . . . . . . . . . . . . . . . . . 2

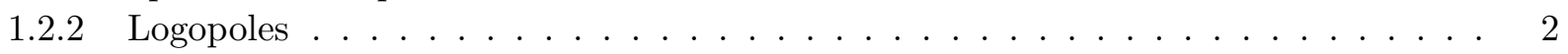

1.2 .3 Point source interaction with sphere $\ldots \ldots \ldots \ldots \ldots \ldots \ldots$

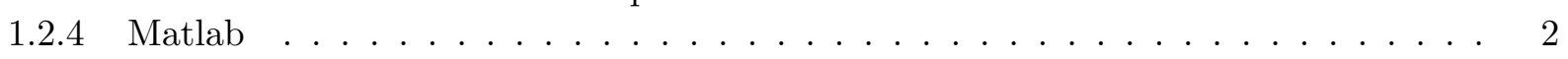

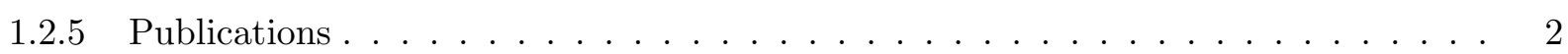

2 Spherical and spheroidal harmonics - definitions and new relations 3

2.1 Laplace's equation in spherical coordinates $\ldots \ldots \ldots \ldots \ldots$

2.1 .1 Solid spherical harmonics $\ldots \ldots \ldots \ldots \ldots \ldots \ldots$

2.1 .2 Translation relations $\ldots \ldots \ldots \ldots \ldots \ldots \ldots \ldots$

2.2 Prolate spheroidal coordinates and harmonics $\ldots \ldots \ldots \ldots \ldots \ldots$

$2.3 \quad$ Green's function expansions $\ldots \ldots \ldots \ldots \ldots \ldots$

2.4 Relations between spherical and spheroidal harmonics $\ldots \ldots \ldots \ldots \ldots$

$3 \quad$ Logopoles - A new class of solutions to Laplace's equation 12

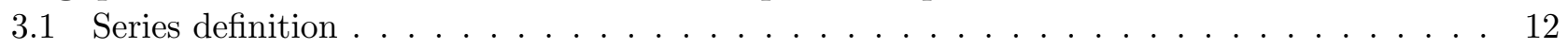

3.2 Expression in terms of offset multipoles of the second kind $\ldots \ldots \ldots \ldots$. . . . . . . 12

3.3 Alternative form of logopoles $\ldots \ldots \ldots \ldots \ldots \ldots \ldots$

3.4 Expression in terms of offset multipoles of the first kind $\ldots \ldots \ldots \ldots \ldots$

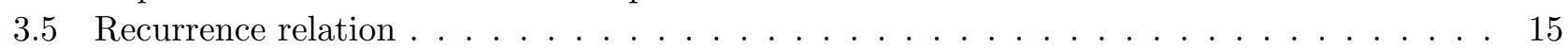

3.6 Lowest orders of logopoles $\ldots \ldots \ldots \ldots \ldots$. . . . . . . . . . . . . . . . 17

3.7 Differentiation and integration along $z$ axis $\ldots \ldots \ldots \ldots \ldots \ldots \ldots$

3.8 Link to spheroidal harmonics $\ldots \ldots \ldots \ldots \ldots \ldots \ldots \ldots$

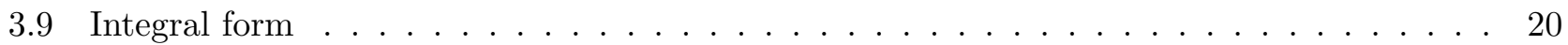

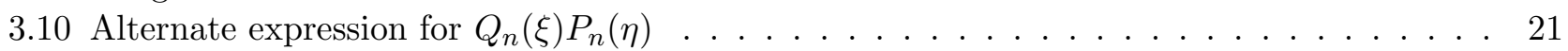

3.11 Attempt at expanding solid harmonics with logopoles . . . . . . . . . . . . . . . . . 22

3.12 Symmetric and antisymmetric logopoles $\ldots \ldots \ldots \ldots \ldots \ldots$

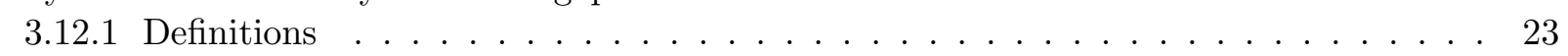

3.12 .2 Spheroidal harmonics as a sum of anti/symmetric logopoles . . . . . . . . . . . . 23

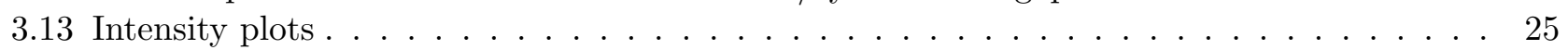

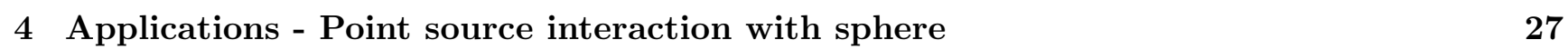

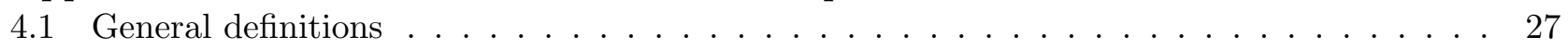

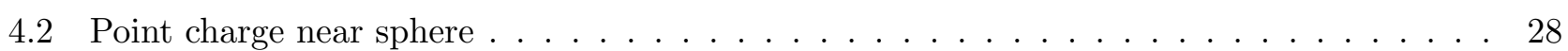

$4.2 .1 \quad$ Standard solutions using spherical harmonics . . . . . . . . . . . . . . . . 28

4.2 .2 Separation of image sources $\ldots \ldots \ldots \ldots \ldots \ldots \ldots \ldots$

4.2 .3 New solution using spheroidal harmonics $\ldots \ldots \ldots \ldots \ldots$. . . . . . . . . . . . . 29

4.2 .4 Image line charge density $\ldots \ldots \ldots \ldots \ldots \ldots \ldots \ldots$

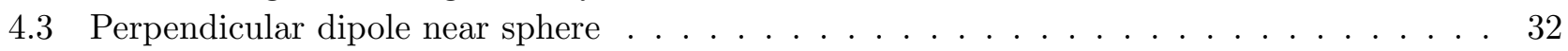

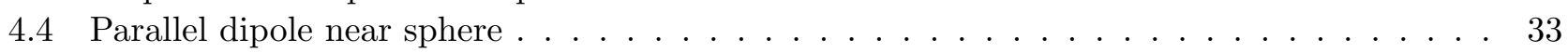

$4.5 \quad$ Reflected electric field and dipole decay rates $\ldots \ldots \ldots \ldots \ldots$. . . . . . . . . . . . . . . . . . . . .

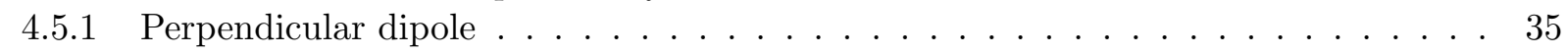




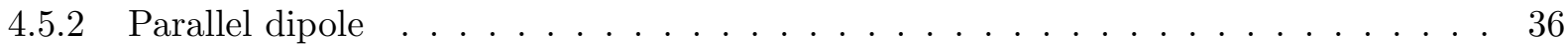

4.6 Potentials inside the sphere $\ldots \ldots \ldots \ldots \ldots \ldots$. . . . . . . . . . . . . 37

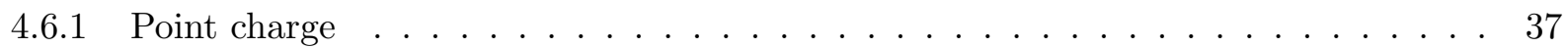

4.6 .2 Perpendicular dipole $\ldots \ldots \ldots \ldots$. . . . . . . . . . . . . . . . . . . . 38

4.6 .3 Parallel dipole $\ldots \ldots \ldots \ldots \ldots \ldots$

4.7 Sources inside the sphere $\left(R_{P}<a\right) \ldots \ldots \ldots \ldots \ldots \ldots \ldots \ldots$

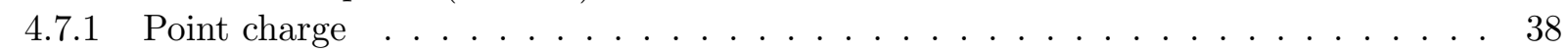

4.7 .2 Perpendicular dipole $\ldots \ldots \ldots \ldots$. . . . . . . . . . . . . . . . . . . . . . 39

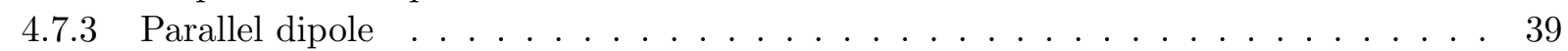

4.8 Logopole solutions $\ldots \ldots \ldots \ldots$. . . . . . . . . . . . . . . . . . . . . . . . . . . . 40

$4.8 .1 \quad$ Potential of point charge near sphere . . . . . . . . . . . . . . . . . . 40

$4.8 .2 \quad$ Perpendicular dipole near sphere . . . . . . . . . . . . . . . . . . . . . 42

4.8 .3 Parallel dipole near sphere . . . . . . . . . . . . . . . . . . . . . 42

5 Generalization to Helmholtz 43

5.1 Generalisation of spherical-spheroidal harmonic expansions $\ldots \ldots \ldots \ldots$

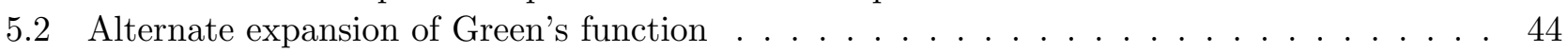

5.3 Expanding acoustic pressure with new functions $\ldots \ldots \ldots \ldots \ldots$

\begin{tabular}{|llr}
\hline & Conclusions and outlook & 47
\end{tabular}

6.1 Relationships for spheroidal harmonics . . . . . . . . . . . . . . . . . . . . . . . 47

6.2 Interacting dielectric spheres $\ldots \ldots \ldots \ldots \ldots \ldots \ldots \ldots$

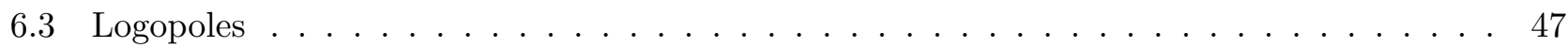

\begin{tabular}{|ll}
\hline Appendices & 48
\end{tabular}

A Proof of Eq. $3.68 \ldots \ldots \ldots \ldots \ldots \ldots \ldots \ldots \ldots \ldots$

B Proof of equation $4.20 \ldots \ldots \ldots \ldots \ldots \ldots \ldots \ldots$

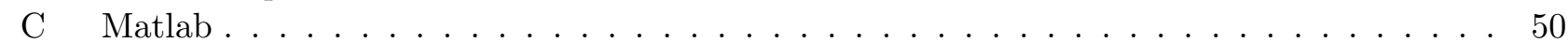

D $\quad$ Numerical instability . . . . . . . . . . . . . . . . . . . . . . 50

E Computation of Legendre functions $\ldots \ldots \ldots \ldots \ldots \ldots \ldots$

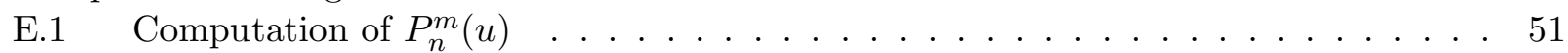

E.2 Computation of $Q_{n}^{m}(u)$ for $|u|>1 \ldots \ldots \ldots \ldots \ldots$. . . . . . . . . . . . . . . 


\section{Chapter 1}

\section{Introduction}

Laplace's equation appears in many areas of physics including electrostatics, gravity, thermodynamics and fluid dynamics. We work with general formulae that could be applied to any of these contexts, but for applications we focus on electrostatics. This thesis investigates new relationships between solutions to Laplace's equation, defines a new class of solutions, and applies these relationships and solutions to problems involving a point source interacting with a dielectric sphere. It also attempts to generalise these relationships to the Helmholtz equation.

\subsection{Background}

Solid spherical harmonics 1 are widely used as a basis of functions to model physical potentials satisfying Laplace's equation, involving point sources or particles with or without spherical symmetry. For elongated objects it is beneficial to use a basis of solid prolate spheroidal harmonics instead; the prolate geometry is stretched in one direction. Naturally one may want to switch between these two bases, and there exist relationships that express one harmonic as a sum of the other type for this. There are four relationships - two between the regular spherical and regular spheroidal harmonics (regular meaning the functions are finite at the origin), and two for the irregular harmonics. The regular relationships were first presented in 1975 [1], and the irregular realtionships were first presented in 2000 [2]. They are also useful for converting functions and solutions between coordinate systems and deriving translation relations for spheroidal harmonics [2], which can then be used for dealing with interactions between multiple spheroidal particles [3].

The problem of a point source interacting with a sphere is fundamental and applicable to many areas of physics. In the case of a point charge outside a conducting sphere, the problem is simple and was first solved by Lord Kelvin in 1845 [4] using an image charge. For the dielectric case the standard solution was first presented by Stratton in 1941 [5], but the problem has continued to be researched until recently because it is fundamental and the standard solution contains a series which converges slowly near the sphere. There has been investigation into expressing the potential using image charges [6] - [9], but these solutions are not straightforward as they involve integrals over image sources.

The Helmholtz equation can be seen as the extension of Laplace's equation from static to harmonic time dependence. The analogue of the solid spherical harmonics are the spherical wave functions, which are the standard basis functions for solving problems involving electromagnetic radiation and scattering by particles. For simple geometrical problems (eg. scattering by a prolate spheroid), series solutions of spherical wave functions may diverge or have slow convergence [10], just as do series of spherical harmonics. For the spherical wave functions, the problem of slow convergence is worse because they cannot always be computed to high orders. Alternate solutions to the Helmholtz equation, for example the spheroidal wave functions, have been widely studied as an alternative basis of for expressing fields, but they are even more problematic as their differential equations involve transcendental numbers and they do not have a simple orthogonality property. One of the main motivations behind the project is

\footnotetext{
${ }^{1}$ We will often omit the word solid for simplicity. The term "spherical harmonic" is commonly used to refer only to the angular part of the full solution to Laplace's equation, and the term "spheroidal harmonic" similarly refers to only the dependence of one spheroidal coordinate of the solution, but we use these terms to mean the full solutions.
} 
to find alternative functions for problems that are poorly modelled by spherical wave functions. But we mostly work with Laplace's equation here because it is simpler and we can work with the underlying concepts before extending them to the Helmholtz equation.

\subsection{Overview}

\subsubsection{Spherical and spheroidal harmonics}

Chapter 2 provides new relationships between prolate spheroidal harmonics and solid spherical harmonics, similar to the known relationships described above. For the known relationships the spheroidal coordinates have their two foci either side of the origin; we derive new similar relationships for when one of the foci is at the origin - when the spheroidal geometry is offset by half its focal length (distance between the foci).

\subsubsection{Logopoles}

Chapter 3 defines and investigates a new infinite class of solutions to Laplace's equation called logopoles. They have several equivalent definitions - they can be written as a finite sum of spheroidal harmonics, as a series of irregular spherical harmonics, as a series of offset spherical irregular harmonics, a sum of spherical harmonics of the second kind (these functions are often discarded for physical applications; their angular part are the Legendre functions of the second kind). Logopoles can be calculated by recurrence and have an integral form. They were actually the main inspiration for the project because they could be applied to the problem of a point source near a sphere. We also use them to derive a relationship between spheroidal harmonics and spherical harmonics of the second kind.

\subsubsection{Point source interaction with sphere}

Chapter 4 focuses on various series solutions for the potential created by different point sources near a sphere. It demonstrates the use of one of the new relationships between spherical and spheroidal harmonics to convert the standard series solutions (involving spherical harmonics) into series comprised of offset spheroidal harmonics. We then show that these series converge much faster near the sphere. We deal with various point sources - a monopole and two orientations of dipoles, both inside and outside the sphere, and find spheroidal harmonic solutions for the internal and external potentials in each case. We also solve some of these problems with logopoles. The logopole series solutions also converge quickly but they are more complicated.

The reflected electric field of a dipole near a sphere is particularly important to nano optics [9]. An oscillating electric dipole has a temporal decay rate which depends on the self induced electric field (the scattered field from the sphere at the dipole position). The electric potential of an oscillating dipole obeys the Helmholtz equation, which can be approximated by Laplace's equation when the wavelength of the light is much longer than the size of the sphere. The electrostatic field provides a first order approximation. We calculate the electrostatic field from the gradient of the potential using both the standard spherical solution and the spheroidal harmonic solution. The quasi-static decay rate computed with spheroidal harmonics converges much faster than with spherical harmonics.

\subsubsection{Matlab}

All important results throughout this thesis are proved analytically, but we used Matlab to search for many relationships and check every identity. In the appendix we briefly describe how Matlab was used.

\subsubsection{Publications}

Part of chapter 4 is currently under review in Physical Review E, under the title "Spheroidal harmonic expansions for the solution of Laplace's equation for a point source near a sphere", with authors Matt Majic, Baptiste Auguié, and Eric C. Le Ru. It is currently available on Arxiv.

I also intend to publish results from chapters 2 and 3 in early 2017. 


\section{Chapter 2}

\section{Spherical and spheroidal harmonics - definitions and new relations}

This thesis uses many different harmonics (solutions to Laplace's equation) and coordinate frames and in this section we define most functions and coordinates for reference, then derive new relationships between these harmonics.

\subsection{Laplace's equation in spherical coordinates}

We use spherical coordinates and harmonics extensively in this thesis. Laplace's equation $\nabla^{2} \psi=0$ is separable in spherical coordinates $(r, \theta, \phi) . \psi$ can be written as a product of three independent functions $\psi=R(r) \Theta(\theta) \Phi(\phi)$ where each of $R, \Theta, \Phi$ obey a second order ordinary differential equation.

The Legendre functions are the solutions to the $\theta$ dependence of spherical harmonics, and they also appear in spheroidal harmonics. Let $f(u)$ where $u=\cos \theta$ be the $\theta$ dependence of $\psi$. It can be shown that $f(u)$ obeys the Legendre differential equation:

$$
\frac{\mathrm{d}}{\mathrm{d} u}\left[\left(1-u^{2}\right) \frac{\mathrm{d} f(u)}{\mathrm{d} u}\right]+\left[n(n+1)-\frac{m^{2}}{1-u^{2}}\right] f(u)=0
$$

$n$ is a non-negative integer. $m$ can be any integer, but typically $|m| \leq n$ for physical solutions (all separable solutions in spherical coordinates with $m>n$ have an infinite line singularity which makes them impractical), and in this thesis we only use $m \geq 0$ (although it is common for authors to include definitions for $m<0$ ). The solutions to this equation are the associated Legendre functions of the first and second kind, $P_{n}^{m}(u)$ and $Q_{n}^{m}(u) . P_{n}^{m}(u)$ are far more commonly used in physical situations since $Q_{n}^{m}(u)$ are singular at $u= \pm 1$ (the entire $z$ axis). Most of this thesis considers problems with azimuthal symmetry (symmetry of revolution about the $z$ axis) corresponding to $m=0$. The first few orders for these functions are (we drop the superscript for $m=0$ ):

$$
\begin{aligned}
P_{0}(u)=1 ; & Q_{0}(u) & =\frac{1}{2} \ln \left|\frac{u+1}{u-1}\right| \\
P_{1}(u)=u ; & Q_{1}(u) & =u Q_{0}(u)-1 \\
P_{2}(u)=\frac{1}{2}\left(3 u^{2}-1\right) ; & Q_{2}(u) & =\frac{1}{2}\left[\left(3 u^{2}-1\right) Q_{0}(u)-3 u\right] \\
P_{3}(u)=\frac{1}{2}\left(5 u^{3}-3 u\right) ; & Q_{3}(u) & =\frac{1}{2}\left[\left(5 u^{3}-3 u\right) Q_{0}(u)-\left(5 u^{2}-\frac{4}{3}\right)\right]
\end{aligned}
$$

And the rest can be calculated by recurrence (Eq. 2.3).

The associated Legendre functions are defined from the $m=0$ Legendre functions as

$$
P_{n}^{m}(u)=\left|1-u^{2}\right|^{m / 2} \frac{\mathrm{d}^{m}}{\mathrm{~d} u^{m}} P_{n}(u), \quad Q_{n}^{m}(u)=\left|1-u^{2}\right|^{m / 2} \frac{\mathrm{d}^{m}}{\mathrm{~d} u^{m}} Q_{n}(u)
$$

These functions for a general $m$ must be used when there is no rotational symmetry of the physical problem. Some authors include a factor of $(-)^{m}$ (short for $\left.(-1)^{m}\right)$ in the definition for $|u| \leq 1$, but this 
factor would have almost no effect on our formulae anyway.

$P_{n}^{m}(u)$ and $Q_{n}^{m}(u)$ both follow the recurrence relation [11] (where $X_{n}^{m}=P_{n}^{m}$ or $Q_{n}^{m}$ ):

$$
(n-m+1) X_{n+1}^{m}(u)=(2 n+1) u X_{n}^{m}(u)-(n+m) X_{n-1}^{m}(u)
$$

and the differential relations:

$$
\begin{aligned}
\left(1-u^{2}\right) \frac{\mathrm{d}}{\mathrm{d} u} X_{n}^{m}(u) & =-n u X_{n}^{m}(u)+(n+m) X_{n-1}^{m}(u) \\
& =(n+1) u X_{n}^{m}(u)-(n-m+1) X_{n-1}^{m}(u) .
\end{aligned}
$$

The first few orders for $m=1$ are:

$$
\begin{aligned}
P_{0}^{1}(u) & =0 & Q_{0}^{1}(u) & =\frac{1}{\sqrt{\left|1-u^{2}\right|}} \\
P_{1}^{1}(u) & =\sqrt{\left|1-u^{2}\right|} & Q_{1}^{1}(u) & =\sqrt{\left|1-u^{2}\right|}\left[Q_{0}^{0}(u)+\frac{u}{1-u^{2}}\right] \\
P_{2}^{1}(u) & =3 u \sqrt{\left|1-u^{2}\right|} & Q_{2}^{1}(u) & =\sqrt{\left|1-u^{2}\right|}\left[3 u Q_{0}^{0}(u)+\frac{3 u^{2}-2}{1-u^{2}}\right] \\
P_{3}^{1}(u) & =\frac{3}{2}\left(5 u^{2}-1\right) \sqrt{\left|1-u^{2}\right|} & & Q_{3}^{1}(u)=\sqrt{\left|1-u^{2}\right|}\left[\frac{3}{2}\left(5 u^{2}-1\right) Q_{0}^{0}(u)+\frac{1}{2} \frac{15 u^{3}-13 u}{1-u^{2}}\right]
\end{aligned}
$$

\subsubsection{Solid spherical harmonics}

The solid spherical harmonics are commonly used as a basis for solutions to Laplace's equation. They depend on all three spherical coordinates, but the $\phi$ dependence is simple $\left(e^{ \pm i m \phi}\right)$ and we can omit it from all our equations without affecting the results. Ignoring the $\phi$ dependence there are a total of four solutions to Laplace's equation in spherical coordinates for each $n$ and $m$ (there are two solutions for each second order ordinary differential equation). However, we mostly work with the following two - the regular solid harmonics: $\left(\frac{r}{R}\right)^{n} P_{n}^{m}(\cos \theta)$, and the irregular solid harmonics: $\left(\frac{R}{r}\right)^{n+1} P_{n}^{m}(\cos \theta)$. In the logopoles chapter we will also use the following functions which are rarely mentioned in literature, we call them regular solid harmonics of the second kind and define them as (we only deal with $m=0$ ): $(-)^{n+1}\left(\frac{r}{R}\right)^{n} Q_{n}(\cos \theta)$. We include the fixed 'radius' $R$ to simplify later expressions and make things non-dimensional. We will use this $R$ in other definitions (coordinate systems and spheroidal harmonics) as a place holder for some distance; it is not necessarily the same $R$ as here unless in the same equation. The regular solid harmonics are finite everywhere, but increase with $r$ so are not useful for fitting solutions that decay far from the origin. The irregular harmonics are singular at the origin, but can be used to describe physical solutions in regions that do not contain the origin. The harmonics of the second kind are almost never used for physical applications since they are singular on the entire $z$ axis. In fact we do not directly use them for physical applications either, but find some relationships between them and other harmonics. We will use the following properties that take the solid harmonics up or down by one order:

$$
\begin{aligned}
& R \frac{\partial}{\partial z}\left[\left(\frac{R}{r}\right)^{n+1} P_{n}(\cos \theta)\right]=-(n+1)\left(\frac{R}{r}\right)^{n+2} P_{n+1}(\cos \theta) \\
& R \frac{\partial}{\partial z}\left[\left(\frac{r}{R}\right)^{n} \begin{array}{ll}
P_{n}(\cos \theta) \\
Q_{n}(\cos \theta)
\end{array}\right]=n\left(\frac{r}{R}\right)^{n-1} \quad \begin{array}{ll}
P_{n-1}(\cos \theta) & Q_{n-1}(\cos \theta)
\end{array}
\end{aligned}
$$

These can be derived using the multivariable differentiation relation $\frac{\partial}{\partial z}=\frac{1}{x} \frac{\partial}{\partial r}+\frac{\sin ^{2} \theta}{r} \frac{\partial}{\partial \cos \theta}$, and relations for $P_{n}$.

\subsubsection{Translation relations}

It is a useful tool to be able to express a solid spherical harmonic in terms of harmonics in a translated coordinate frame. We define two translated frames, centred at $\mathrm{O}^{\prime}$ and O", offset by $+R$ and $-R$ respectively along the $z$-axis (see figure 2.1). Notation: we use $R$, O' and $\mathrm{O}$ " for general formulas, but not for 
the applications section (there $R \rightarrow R_{I}$ and $\mathrm{O}^{\prime} \rightarrow \mathrm{P}$ ). The new coordinates can be expressed as:

$$
\left\{\begin{array} { l l } 
{ x ^ { \prime } } & { = x } \\
{ y ^ { \prime } } & { = y } \\
{ \rho ^ { \prime } } & { = \rho = \sqrt { x ^ { 2 } + y ^ { 2 } } } \\
{ z ^ { \prime } } & { = z - R } \\
{ r ^ { \prime } } & { = \sqrt { \rho ^ { 2 } + ( z - R ) ^ { 2 } } } \\
{ \operatorname { c o s } \theta ^ { \prime } } & { = z ^ { \prime } / r ^ { \prime } }
\end{array} \quad \left\{\begin{array}{ll}
x^{\prime \prime} & =x \\
y^{\prime \prime} & =y \\
\rho^{\prime \prime} & =\rho \\
z^{\prime \prime} & =z+R \\
r^{\prime \prime} & =\sqrt{\rho^{2}+(z+R)^{2}} \\
\cos \theta^{\prime \prime} & =z^{\prime \prime} / r^{\prime \prime}
\end{array}\right.\right.
$$

Harmonics in the primed frame can be written as a sum or series of harmonics centred at the origin:

$$
\begin{array}{rlrl}
\left(\frac{r^{\prime}}{R}\right)^{n} P_{n}\left(\cos \theta^{\prime}\right) & =\sum_{k=0}^{n}(-)^{n+k}\left(\begin{array}{l}
n \\
k
\end{array}\right)\left(\frac{r}{R}\right)^{k} P_{k}(\cos \theta), & \text { where }\left(\begin{array}{l}
n \\
k
\end{array}\right)=\frac{n !}{(n-k) ! k !} \\
\left(\frac{R}{r^{\prime}}\right)^{n+1} P_{n}\left(\cos \theta^{\prime}\right) & =\sum_{k=n}^{\infty}\left(\begin{array}{c}
k \\
n
\end{array}\right)\left(\frac{R}{r}\right)^{k+1} P_{k}(\cos \theta) & & (r>R) \\
& =(-)^{n} \sum_{k=0}^{\infty}\left(\begin{array}{c}
k+n \\
n
\end{array}\right)\left(\frac{r}{R}\right)^{k} P_{k}(\cos \theta) & & (r<R)
\end{array}
$$

These relationships are specific cases of formulae in [12], but for a hint at their derivation:

Eq. 2.9 can be obtained by assuming some expansion coefficients $\alpha_{n}^{k}$, and evaluating the expansion at $\cos \theta=\cos \theta^{\prime}=1$. Then the expansion reduces to $\left(r^{\prime} / R\right)^{n}=(r / R-1)^{n}=\sum_{k=0}^{n} \alpha_{n}^{k}(r / R)^{k}$, and it is clear that $\alpha_{n}^{k}$ must be binomial coefficients (up to a sign).

For Eq. 2.10, the case for $n=0$ comes directly from the generating function for the Legendre polynomials: $\frac{1}{\sqrt{1-2 u t+t^{2}}}=\sum_{k=0}^{\infty} t^{n} P_{n}(u)$ with $t=r / R$ and $u=\cos \theta$. The formula for general $n$ can be obtained by differentiating both sides $n$ times with respect to $z$ and applying Eq. 2.6.

For Eq. 2.11, the case for $n=0$ comes from the generating function, this time with $t=R / r$. Differentiate both sides $n$ times with respect to $z$ and apply Eqs. 2.6 and 2.7 .

\subsection{Prolate spheroidal coordinates and harmonics}

We will present new relationships between spherical and spheroidal harmonics, and new solutions to problems using spheroidal harmonics (these will use an unconventional offset coordinate frame, but first we will define spheroidal coordinates in the conventional frame - we will also present some known results in the standard frame for comparison). Prolate spheroidal coordinates are defined using two focal points on the $z$ axis at $z=R$ and $-R$. There are other definitions for prolate spheroidal coordinates but we use $(\xi, \eta, \phi)$, where $\xi$ is the normalised average of the distance from two focal points (on the $z$ axis), $\eta$ is the normalised difference of these distances and $\phi$ is the same as in spherical coordinates:

$$
\xi=\frac{r^{\prime \prime}+r^{\prime}}{2 R}=\frac{\sqrt{r^{2}+2 R z+R^{2}}+\sqrt{r^{2}-2 R z+R^{2}}}{2 R}, \quad \eta=\frac{r^{\prime \prime}-r^{\prime}}{2 R}
$$

Surfaces of constant $\xi$ are spheroids and surfaces of constant $\eta$ are paraboloids. For background we will now sketch a derivation of the forms of prolate spheroidal harmonics. The Laplacian is [13]

$$
\nabla^{2} \psi=\frac{1}{R^{2}\left(\xi^{2}-\eta^{2}\right)}\left[\frac{\partial}{\partial \xi}\left(\xi^{2}-1\right) \frac{\partial \psi}{\partial \xi}+\frac{\partial}{\partial \eta}\left(1-\eta^{2}\right) \frac{\partial \psi}{\partial \eta}+\frac{\xi^{2}-\eta^{2}}{\left(\xi^{2}-1\right)\left(1-\eta^{2}\right)} \frac{\partial^{2} \psi}{\partial \phi^{2}}\right]
$$

so Laplace's equation is

$$
\frac{\partial}{\partial \xi}\left(\xi^{2}-1\right) \frac{\partial \psi}{\partial \xi}+\frac{\partial}{\partial \eta}\left(1-\eta^{2}\right) \frac{\partial \psi}{\partial \eta}+\left(\frac{1}{\xi^{2}-1}+\frac{1}{1-\eta^{2}}\right) \frac{\partial^{2} \psi}{\partial \phi^{2}}=0
$$

Laplaces equation is separable in prolate spheroidal coordinates. To find separable solutions, assume that $\psi=\Xi(\xi) H(\eta) \Phi(\phi)$ and insert this into Eq. 2.13 and divide by $\psi$ :

$$
\frac{1}{\Xi} \frac{\partial}{\partial \xi}\left(\xi^{2}-1\right) \frac{\partial \Xi}{\partial \xi}+\frac{1}{H} \frac{\partial}{\partial \eta}\left(1-\eta^{2}\right) \frac{\partial H}{\partial \eta}+\frac{1}{\Phi}\left(\frac{1}{\xi^{2}-1}+\frac{1}{1-\eta^{2}}\right) \frac{\partial^{2} \Phi}{\partial \phi^{2}}=0
$$


Differentiating this with respect to $\phi$, we obtain:

$$
\frac{\partial}{\partial \phi}\left(\frac{1}{\Phi} \frac{\partial^{2} \Phi}{\partial \phi^{2}}\right)=0 \Rightarrow \frac{1}{\Phi} \frac{\partial^{2} \Phi}{\partial \phi^{2}}=a
$$

Technically $a$ could be an arbitrary constant, but it must equal $-m^{2}$ where $m$ is an integer to satisfy that $\Phi(\phi+2 \pi)=\Phi(\phi)$; the solutions are $\Phi=e^{ \pm i m \phi}$. Then we have $\Phi^{\prime \prime} / \Phi=-m^{2}$ and can insert this into Eq. 2.14:

$$
\begin{aligned}
\frac{1}{\Xi} \frac{\partial}{\partial \xi}\left(\xi^{2}-1\right) \frac{\partial \Xi}{\partial \xi}+\frac{1}{H} \frac{\partial}{\partial \eta}\left(1-\eta^{2}\right) \frac{\partial H}{\partial \eta}-m^{2}\left(\frac{1}{\xi^{2}-1}+\frac{1}{1-\eta^{2}}\right) & =0 \\
\Rightarrow \frac{1}{\Xi} \frac{\partial}{\partial \xi}\left(\xi^{2}-1\right) \frac{\partial \Xi}{\partial \xi}-\frac{m^{2}}{\xi^{2}-1}=b ; & \frac{1}{H} \frac{\partial}{\partial \eta}\left(1-\eta^{2}\right) \frac{\partial H}{\partial \eta}-\frac{m^{2}}{1-\eta^{2}}=-b
\end{aligned}
$$

for an arbitrary constant $b$. In order to have solutions which are finite at $\eta= \pm 1$, we must have $b=-n(n+1)$ where $n$ is a positive integer. Then Eqs. 2.17 both become Legendre's equation, and $P_{n}^{m}(\xi) P_{n}^{m}(\eta), Q_{n}^{m}(\xi) P_{n}^{m}(\eta)$, $P_{n}^{m}(\xi) Q_{n}^{m}(\eta), Q_{n}^{m}(\xi) Q_{n}^{m}(\eta)$ are all solutions. However only two are commonly used - the 'regular': $P_{n}^{m}(\xi) P_{n}^{m}(\eta)$ and 'irregular': $Q_{n}^{m}(\xi) P_{n}^{m}(\eta)$, because the others contain infinite line singularities.

$Q_{n}^{m}(\xi) P_{n}^{m}(\eta)$ go to zero as $r \rightarrow \infty$ and are singular on the $z$-axis between $z=-R$ and $z=R . P_{n}^{m}(\xi) P_{n}^{m}(\eta)$ are regular everywhere and diverge as $r \rightarrow \infty$. We call the 'focal length' of the spheroidal harmonics the distance between the origins of $\mathrm{O}$ ' and $\mathrm{O}$ " used to define $\xi$ and $\eta$.

We also define offset spheroidal coordinates $(\bar{\xi}, \bar{\eta}, \phi)$ where

$$
\bar{\xi}=\frac{r+r^{\prime}}{R}, \quad \bar{\eta}=\frac{r-r^{\prime}}{R} .
$$

These have the same shape as the non-barred coordinates, but are scaled down by 2 and translated

Figure 2.1: Schematic of some of the coordinates used in this thesis for general formulae up the $z$-axis by $R / 2$, as seen in figure 2.1. We find new relationships between the harmonics $P_{n}^{m}(\bar{\xi}) P_{n}^{m}(\bar{\eta})$ and $Q_{n}^{m}(\bar{\xi}) P_{n}^{m}(\bar{\eta})$ and solid spherical harmonics in section ??. We also use these spheroidal harmonics in an offset frame as solutions to the problem of a point source near a sphere in section 4 .

\subsection{Green's function expansions}

We present the expansion of Green's function in terms of different harmonics - we use these expansions for the proofs of some relations between spherical and spheroidal harmonics. Below are two known expansions of Green's function for points $\mathbf{r}_{1}=\left(r_{1}, \theta_{1}, \phi_{1}\right) \equiv\left(\xi_{1}, \eta_{1}, \phi_{1}\right)$ and $\mathbf{r}_{2}=\left(r_{2}, \theta_{2}, \phi_{2}\right) \equiv\left(\xi_{2}, \eta_{2}, \phi_{2}\right)$ [2]. The spherical harmonic expansion which applies for $r_{1}<r_{2}$ :

$$
\frac{1}{\left|\mathbf{r}_{\mathbf{1}}-\mathbf{r}_{\mathbf{2}}\right|}=\sum_{k=0}^{\infty} \frac{r_{1}^{k}}{r_{2}^{k+1}} \sum_{m=0}^{k}\left(2-\delta_{m 0}\right) \frac{(k-m) !}{(k+m) !} P_{k}^{m}\left(\cos \theta_{1}\right) P_{k}^{m}\left(\cos \theta_{2}\right) \cos m\left(\phi_{1}-\phi_{2}\right) \text {. }
$$


Note that for $\mathbf{r}_{1}$ or $\mathbf{r}_{2}$ on the $z$-axis, Eq. 2.18 reduces to the translation relation Eq. 2.10 for $n=0$. And in terms of spheroidal harmonics with $\xi_{1}<\xi_{2}$ :

$$
\frac{1}{\left|\mathbf{r}_{1}-\mathbf{r}_{\mathbf{2}}\right|}=\sum_{n=0}^{\infty} \frac{2 n+1}{R} \sum_{m=0}^{n}(-)^{m}\left(2-\delta_{m 0}\right) \frac{(n-m) !^{2}}{(n+m) !^{2}} P_{n}^{m}\left(\xi_{1}\right) P_{n}^{m}\left(\eta_{1}\right) Q_{n}^{m}\left(\xi_{2}\right) P_{n}^{m}\left(\eta_{2}\right) \cos m\left(\phi_{1}-\phi_{2}\right),
$$

where $R$ is half the focal length of the spheroidal harmonics. However, we will need the expansion of Green's function in terms of the offset "barred" spheroidal harmonics, which can be obtained by coordinate changing Eq. 2.19 into barred-spheroidal coordinates with $\bar{\xi}_{1}<\bar{\xi}_{2}$ :

$$
\frac{1}{\left|\mathbf{r}_{\mathbf{1}}-\mathbf{r}_{\mathbf{2}}\right|}=\sum_{n=0}^{\infty} 2 \frac{2 n+1}{R} \sum_{m=0}^{n}(-)^{m}\left(2-\delta_{m 0}\right) \frac{(n-m) !^{2}}{(n+m) !^{2}} P_{n}^{m}\left(\bar{\xi}_{1}\right) P_{n}^{m}\left(\bar{\eta}_{1}\right) Q_{n}^{m}\left(\bar{\xi}_{2}\right) P_{n}^{m}\left(\bar{\eta}_{2}\right) \cos m\left(\phi_{1}-\phi_{2}\right)
$$

The coordinate change involves two steps, first translating up the $z$-axis by $R / 2$, (the coordinate change applies to both points $\mathbf{r}_{\mathbf{1}}$ and $\mathbf{r}_{\mathbf{2}}$ ) this does not change the left hand side since the distance between the points is unaffected by a translated frame. Then scale coordinates by half; this halves the distance between $\mathbf{r}_{\mathbf{1}}$ and $\mathbf{r}_{\mathbf{2}}$, and finishes the conversion to barred spheroidal coordinates.

\subsection{Relations between spherical and spheroidal harmonics}

We first present the four known relations between the regular and irregular spherical solid harmonics, and the regular and irregular prolate spheroidal harmonics. The azimuthal dependence $e^{ \pm i m \phi}$ is omitted since it is the same on both sides.

$$
\begin{aligned}
P_{n}^{m}(\xi) P_{n}^{m}(\eta) & =\sum_{\substack{k=m \\
n+k \text { even }}}^{n} \frac{(-)^{(n-k) / 2}(n+k-1) ! !}{(n-k) ! !(k+m) !} \frac{(n+m) !}{(n-m) !}\left(\frac{r}{R}\right)^{k} P_{k}^{m}(\cos \theta) \\
\left(\frac{r}{R}\right)^{n} P_{n}^{m}(\cos \theta) & =\sum_{\substack{k=m \\
n+k \text { even }}}^{n} \frac{(2 k+1)(n+m) !}{(n-k) ! !(n+k+1) ! !} \frac{(k-m) !}{(k+m) !} P_{k}^{m}(\xi) P_{k}^{m}(\eta) \\
Q_{n}^{m}(\xi) P_{n}^{m}(\eta)= & \sum_{\substack{k=n \\
n+k \text { even }}}^{\infty} \frac{(-)^{m}(k-m) !}{(k-n) ! !(k+n+1) ! !} \frac{(n+m) !}{(n-m) !}\left(\frac{R}{r}\right)^{k+1} P_{k}^{m}(\cos \theta) \\
\left(\frac{R}{r}\right)^{n+1} P_{n}^{m}(\cos \theta)= & \sum_{\substack{k=n \\
n+k \text { even }}}^{\infty} \frac{(-)^{(n-k) / 2+m}(2 k+1)(n+k-1) ! !}{(k-n) ! !(n-m) !} \frac{(k-m) !}{(k+m) !} Q_{k}^{m}(\xi) P_{k}^{m}(\eta)
\end{aligned}
$$

$n ! ! \equiv n(n-2)(n-4) \ldots$ and $(-1) ! ! \equiv 0 ! ! \equiv 1$. Derivations can be found in [2], [14].

There also exist two more spherical harmonics $r^{n} Q_{n}^{m}(\cos \theta), r^{-n-1} Q_{n}^{m}(\cos \theta)$ and two more spheroidal harmonics $P_{n}^{m}(\xi) Q_{n}^{m}(\eta)$ and $Q_{n}^{m}(\xi) Q_{n}^{m}(\eta)$, but these are not useful due to singularities along the $z$ axis. As far as we have seen there are no known spherical - spheroidal relationships between these except one that we derive in section 3.10 .

In the four expansions above, note that the spherical harmonics are centred half way between the foci of the spheroidal harmonics. Eq. 2.23 is only valid for $r>R$ and Eq. 2.24 is valid everywhere except the singularity of $Q_{n}^{m}(\xi)$ - on the $z$ axis from $z=-R$ to $R$.

Similar to Eqs. 2.21 - 2.24, we now derive new relations for when the spherical and spheroidal coordinate frames are offset from each other so that the spherical harmonics are centred at one of the foci of the spheroidal coordinates. So we can relate the 'barred' spheroidal harmonics to the spherical 
harmonics:

$$
\begin{gathered}
P_{n}^{m}(\bar{\xi}) P_{n}^{m}(\bar{\eta})=\frac{(n+m) !}{(n-m) !} \sum_{k=m}^{n} \frac{(-)^{n+k}}{k !(k+m) !} \frac{(n+k) !}{(n-k) !}\left(\frac{r}{R}\right)^{k} P_{k}^{m}(\cos \theta) \\
\left(\frac{r}{R}\right)^{n} P_{n}^{m}(\cos \theta)=n !(n+m) ! \sum_{k=m}^{n} \frac{2 k+1}{(n-k) !(n+k+1) !} \frac{(k-m) !}{(k+m) !} P_{k}^{m}(\bar{\xi}) P_{k}^{m}(\bar{\eta}) \\
Q_{n}^{m}(\bar{\xi}) P_{n}^{m}(\bar{\eta})=\frac{(-)^{m}}{2} \frac{(n+m) !}{(n-m) !} \sum_{k=n}^{\infty} \frac{k !(k-m) !}{(k-n) !(k+n+1) !}\left(\frac{R}{r}\right)^{k+1} P_{k}^{m}(\cos \theta) \\
\left(\frac{R}{r}\right)^{n+1} P_{n}^{m}(\cos \theta)=\frac{2(-)^{n+m}}{n !(n-m) !} \sum_{k=n}^{\infty}(-)^{k}(2 k+1) \frac{(k+n) !}{(k-n) !} \frac{(k-m) !}{(k+m) !} Q_{k}^{m}(\bar{\xi}) P_{k}^{m}(\bar{\eta})
\end{gathered}
$$

Eq. 2.27 is only valid for $r>R$; the focal length of the barred spheroidal harmonics is half of that for the non-barred harmonics, so the radius of divergence of Eq. 2.27 is effectively twice the size of that for Eq. 2.23. For an expansion of $Q_{n}(\xi) P_{n}(\eta)$ or $Q_{n}(\bar{\xi}) P_{n}(\bar{\eta})$ that is valid in all space, see Eq. 3.56 later. Eq. 2.28 is valid everywhere except the singularity of $Q_{n}^{m}(\bar{\xi})$ - on the $z$ axis from $z=0$ to $R$.

These relations can be coordinate shifted down the $z$-axis by $R / 2$ and scaled up by 2 , so that the barred spheroidal harmonics become non-barred and the solid harmonics become double primed - centred at $(x, y, z)=(0,0,-R)$. We can also expand spheroidal harmonics in terms of spherical harmonics centred at $\mathrm{O}^{\prime}$, by then reflecting coordinates around $z=0$ and using the simple symmetry relations for the Legendre functions, $P_{n}^{m}(-x)=(-)^{n+m} P_{n}^{m}(x)$ and $Q_{n}^{m}(-x)=-(-)^{n+m} Q_{n}(x)$.

\section{Proof of Eq. 2.25}

Consider the expansion of $P_{n}^{m}(\bar{\xi}) P_{n}^{m}(\bar{\eta}) e^{i m \phi}$ in terms of regular solid harmonics $r^{k} P_{k}^{m}(\cos \theta) e^{i m \phi}$, which must exist since the solid harmonics are a basis for regular solutions to Laplace's equation. $m$ must be the same on both sides since $e^{i m \phi}$ are independent functions. So we write:

$$
P_{n}^{m}(\bar{\xi}) P_{n}^{m}(\bar{\eta})=\sum_{k=m}^{\infty} \alpha_{n k}^{m}\left(\frac{r}{R}\right)^{k} P_{k}^{m}(\cos \theta) .
$$

The associated Legendre functions can be written as

$$
P_{n}^{m}(x)=\left|1-x^{2}\right|^{m / 2} \Pi_{n}^{m}(x) \quad \text { with } \Pi_{n}^{m}(x)=\frac{\mathrm{d}^{m}}{\mathrm{~d} x^{m}} P_{n}(x) .
$$

Note $\Pi_{n}^{m}(x)$ is a polynomial of order $n-m$. One can show that the "barred" spheroidal coordinates satisfy $R^{2}\left(1-\bar{\eta}^{2}\right)\left(\bar{\xi}^{2}-1\right)=4 r^{2} \sin ^{2} \theta$, from this we deduce

$$
P_{n}^{m}(\bar{\xi}) P_{n}^{m}(\bar{\eta})=(-)^{m}\left(\frac{2 r}{R}\right)^{m} \sin ^{m} \theta \Pi_{n}^{m}(\bar{\xi}) \Pi_{n}^{m}(\bar{\eta})
$$

so Eq. 2.29 can be written in terms of $\Pi_{n}^{m}$ as

$$
\Pi_{n}^{m}(\bar{\xi}) \Pi_{n}^{m}(\bar{\eta})=\sum_{k=m}^{\infty} \alpha_{n k}^{m}\left(\frac{r}{R}\right)^{k-m} \frac{1}{2^{m}} \Pi_{k}^{m}(\cos \theta)
$$

The coefficients $\alpha_{n k}^{m}$ can be determined by evaluating the expansion at $\theta=0, z>R$, where $\bar{\eta}=1$ and $r=R(\bar{\xi}+1) / 2$. Using the special value:

$$
\Pi_{n}^{m}(1)=\frac{1}{2^{m}} \frac{(n+m) !}{m !(n-m) !}
$$

we obtain

$$
\Pi_{n}^{m}(\bar{\xi})=\sum_{k=m}^{n} \alpha_{n k}^{m} \frac{(k+m) !(n-m) !}{(k-m) !(n+m) !} \frac{1}{2^{m}}\left(\frac{\bar{\xi}+1}{2}\right)^{k-m}
$$


We then derive from scratch the coefficients for the expansion of $\Pi_{n}^{m}(\bar{\xi})$ in terms of $(\bar{\xi}+1) / 2$. To do this, start from the definition of the associated Legendre functions:

$$
\begin{aligned}
P_{n}^{m}(\bar{\xi}) \frac{2^{n} n !}{\sqrt{\bar{\xi}^{2}-1} m} & =\partial_{\bar{\xi}}^{n+m}\left(\bar{\xi}^{2}-1\right)^{n} \\
& =\partial_{\bar{\xi}}^{n+m}(\bar{\xi}+1)^{n}(\bar{\xi}+1-2)^{n} \\
& =\partial_{\bar{\xi}}^{n+m} \sum_{k=0}^{n}\left(\begin{array}{l}
n \\
k
\end{array}\right)(\bar{\xi}+1)^{n+k}(-2)^{n-k} \\
& =\sum_{k=0}^{n}\left(\begin{array}{l}
n \\
k
\end{array}\right) \frac{(n+k) !}{(k-m) !}(\bar{\xi}+1)^{k-m}(-2)^{n-k} \\
\Rightarrow \Pi_{n}^{m}(\bar{\xi}) & =\sum_{k=0}^{n} \frac{(-)^{n-k}}{2^{m}} \frac{(n+k) !}{k !(k-m) !}\left(\frac{\bar{\xi}+1}{2}\right)^{k-m}
\end{aligned}
$$

From this, we identify

$$
\alpha_{n k}^{m}=(-)^{n+k} \frac{(n+m) !}{(n-m) !} \frac{(n+k) !}{k !(n-k) !(k+m) !}, \text { for } m \leq k \leq n, 0 \text { otherwise }
$$

which are the coefficients of Eq. 2.25.

Proof of Eq. 2.26

This proof is long and indirect compared to the previous one. Since $P_{n}^{m}(\bar{\xi}) P_{n}^{m}(\bar{\eta})$ can be written as a finite sum over $k$ of $P_{k}^{m}(\cos \theta)$, we can also write $P_{n}^{m}(\cos \theta)=\sum_{k=m}^{n} \beta_{n k}^{m} P_{k}^{m}(\bar{\xi}) P_{k}^{m}(\bar{\eta})$ (where each $\beta_{n k}^{m}$ is a constant). Evaluate this assumed expansion at $\bar{\eta}=1, \cos \theta=1$ as before with Eq. 2.25 (and relabel $\bar{\xi} \rightarrow x)$. Then the problem reduces to

$$
\frac{(n+m) !}{(n-m) !}\left(x^{2}-1\right)^{m / 2}\left(\frac{x+1}{2}\right)^{n-m}=\sum_{k=m}^{n} 2^{m} \beta_{n k}^{m} \frac{(k+m) !}{(k-m) !} P_{n}^{m}(x)
$$

Essentially we need to show that $\gamma_{n k}^{m} \equiv 2^{n} \frac{(k+m) !(n-m) !}{(k-m) !(n+m) !} \beta_{n k}^{m}$ solves

$$
\left(x^{2}-1\right)^{m / 2}(x+1)^{n-m}=\sum_{k=m}^{n} \gamma_{n k}^{m} P_{k}^{m}(x)
$$

This can be done by finding a recurrence relation on $n$ for $\gamma_{n k}^{m}$ and showing that the assumed expression for $\gamma_{n k}^{m}$ defined from Eq. 2.26 satisfies the recurrence and initial values. Begin with

$$
\begin{aligned}
\left(x^{2}-1\right) \partial_{x}\left[\left(x^{2}-1\right)^{m / 2}(x+1)^{n-m}\right] & =m x\left(x^{2}-1\right)^{m / 2}(x+1)^{n-m}+(n-m)\left(x^{2}-1\right)\left(x^{2}-1\right)^{m / 2}(x+1)^{n-m-1} \\
& =n x\left(x^{2}-1\right)^{m / 2}(x+1)^{n-m}-(n-m)\left(x^{2}-1\right)^{m / 2}(x+1)^{n-m}
\end{aligned}
$$

Plug in the assumed expansion Eq. 2.36.

$$
\left(x^{2}-1\right) \partial_{x} \sum_{k=m}^{n} \gamma_{n k}^{m} P_{k}^{m}=n x \sum_{k=m}^{n} \gamma_{n k}^{m} P_{k}^{m}-(n-m) \sum_{k=m}^{n} \gamma_{n k}^{m} P_{k}^{m}
$$

and use these properties

$$
\begin{aligned}
& x P_{k}^{m}=\frac{(k-m+1) P_{k+1}^{m}+(k+m) P_{k-1}^{m}}{2 k+1} \\
& \left(x^{2}-1\right) \partial_{x} P_{k}^{m}=\frac{k(k-m+1) P_{k+1}^{m}-(k+1)(k+m) P_{k-1}^{m}}{2 k+1}
\end{aligned}
$$


to obtain everything in terms of Legendre functions:

$$
\begin{gathered}
\sum_{k=m}^{n} \gamma_{n k}^{m} \frac{k(k-m+1) P_{k+1}^{m}-(k+1)(k+m) P_{k-1}^{m}}{2 k+1}=n \sum_{k=m}^{n} \gamma_{n k}^{m} \frac{(k-m+1) P_{k+1}^{m}+(k+m) P_{k-1}^{m}}{2 k+1} \\
-(n-m) \sum_{k=m}^{n} \gamma_{n k}^{m} P_{k}^{m} \\
\Rightarrow \sum_{k=m}^{n} \gamma_{n k}^{m} \frac{(n-k)(k-m+1) P_{k+1}^{m}+(k+m)(n+k+1) P_{k-1}^{m}}{2 k+1}=\sum_{k=m}^{n} \gamma_{n k}^{m}(n-m) P_{k}^{m}
\end{gathered}
$$

Re-index the sums so that they are all sums of $P_{k}^{m}$ :

$$
\sum_{k=m+1}^{n+1} \gamma_{n, k-1}^{m} \frac{(n-k+1)(k-m)}{2 k-1} P_{k}^{m}+\sum_{k=m-1}^{n-1} \gamma_{n, k+1}^{m} \frac{(k+m+1)(n+k+2)}{2 k+3} P_{k}^{m}=(n-m) \sum_{k=m}^{n} \gamma_{n k}^{m} P_{k}^{m}
$$

Since the Legendre functions are linearly independent, this must hold for each value of $k$ :

$$
\gamma_{n, k-1}^{m} \frac{(n-k+1)(k-m)}{2 k-1}+\gamma_{n, k+1}^{m} \frac{(k+m+1)(n+k+2)}{2 k+3}=(n-m) \gamma_{n k}^{m}
$$

Now assume that

$$
\gamma_{n k}^{m}=\frac{2^{n}(2 k+1)(n-m) ! n !}{(n-k) !(n+k+1) !}
$$

With this $\gamma_{n k}^{m}$, we have that $\beta_{n k}^{m}$ equal the coefficients in Eq. 2.25. It can be shown that $\gamma_{n k}^{m}$ satisfies Eq. 2.38. It is also straightforward to check that the coefficients of $P_{k}^{m}$ for $k=m-1, m, n, n+1$ in Eq. 2.37 are equal on both sides.

We must also show it has the correct initial values - it is easiest to find $\gamma_{n n}^{m}$, by looking at the coefficient of $x^{n-m}$ in

$$
(x+1)^{n-m}=\sum_{k=m}^{n} \gamma_{n k}^{m} \Pi_{k}^{m}(x)
$$

$\gamma_{n n}^{m}$ must be such that the coefficient of $x^{n-m}$ is the same on both sides. The left hand side coefficient is 1 , and on the right hand side, only the $n^{\text {th }}$ term in the sum contains $x^{n-m}$, and its coefficient can be found from Rodriguez's formula to be $\frac{(2 n) !}{2^{n} n !(n-m) !}$. Therefore $\gamma_{n n}^{m}=\frac{2^{n} n !(n-m) !}{(2 n) !}$ which is consistent with Eq. 2.39.

For the second initial value, we need $\gamma_{n, n-1}^{m}$, which can be found by comparing the coefficients of $x^{n-m-1}$. The 1.h.s. coefficient is $n-m$, and for the r.h.s. only the $(n-1)^{t h}$ term contains $x^{n-m-1}$, with a coefficient of $\frac{(2 n-2) !}{2^{n-1}(n-1) !(n-m-1) !}$. Therefore $\gamma_{n, n-1}^{m}=\frac{2^{n-1}(n-1) !(n-m) !}{(2 n-2) !}$, again consistent with Eq. 2.39. 


\section{Derivation of Eq. 2.28}

To prove Eq. 2.28, we will make use of the expansions of Green's function in terms of both spherical and spheroidal solid harmonics. For points $\mathbf{r}_{1}$ and $\mathbf{r}_{2}$ with $r_{1}<r_{2}$ and $\bar{\xi}_{1}<\bar{\xi}_{2}$ :

$$
\begin{aligned}
\frac{1}{\left|\mathbf{r}_{1}-\mathbf{r}_{2}\right|} & =\sum_{n=0}^{\infty} \frac{r_{1}^{n}}{r_{2}^{n+1}} \sum_{m=0}^{n}\left(2-\delta_{m 0}\right) \frac{(n-m) !}{(n+m) !} P_{n}^{m}\left(\cos \theta_{1}\right) P_{n}^{m}\left(\cos \theta_{2}\right) \cos m\left(\phi_{1}-\phi_{2}\right) \\
& =\sum_{k=0}^{\infty} 2 \frac{2 k+1}{R} \sum_{m=0}^{k}(-)^{m}\left(2-\delta_{m 0}\right) \frac{(k-m) !^{2}}{(k+m) !^{2}} P_{k}^{m}\left(\bar{\xi}_{1}\right) P_{k}^{m}\left(\bar{\eta}_{1}\right) Q_{k}^{m}\left(\bar{\xi}_{2}\right) P_{k}^{m}\left(\bar{\eta}_{2}\right) \cos m\left(\phi_{1}-\phi_{2}\right)
\end{aligned}
$$

Now substitute Eq.2.25 for $\mathbf{r}_{1}$ into Eq.2.41 to express it as an expansion on the same spherical harmonic basis:

$$
\begin{aligned}
& \frac{1}{\left|\mathbf{r}_{1}-\mathbf{r}_{2}\right|}=\sum_{k=0}^{\infty} 2 \frac{2 k+1}{R} \sum_{m=0}^{k}(-)^{m}\left(2-\delta_{m 0}\right) \frac{(k-m) !^{2}}{(k+m) !^{2}}\left(\frac{(k+m) !}{(k-m) !} \sum_{n=m}^{k} \frac{(-)^{k+n}}{n !(n+m) !} \frac{(k+n) !}{(k-n) !} \frac{r_{1}^{n}}{R^{n}} P_{n}^{m}\left(\cos \theta_{1}\right)\right) \\
& \times Q_{k}^{m}\left(\bar{\xi}_{2}\right) P_{k}^{m}\left(\bar{\eta}_{2}\right) \cos m\left(\phi_{1}-\phi_{2}\right) . \\
& =\sum_{n=0}^{\infty} \sum_{m=0}^{n} \sum_{k=n}^{\infty} 2 \frac{2 k+1}{R}\left(2-\delta_{m 0}\right) \frac{(-)^{n+k+m}(k-m) !(k+n) !}{(k+m) !(k-n) ! n !(n+m) !} \frac{r_{1}^{n}}{R^{n}} P_{n}^{m}\left(\cos \theta_{1}\right) Q_{k}^{m}\left(\bar{\xi}_{2}\right) P_{k}^{m}\left(\bar{\eta}_{2}\right) \cos m\left(\phi_{1}-\phi_{2}\right)
\end{aligned}
$$

where we have swapped the order of the sums using first $\sum_{m=0}^{k} \sum_{n=m}^{k}=\sum_{n=0}^{k} \sum_{m=0}^{n}$ and then $\sum_{k=0}^{\infty} \sum_{n=0}^{k}=\sum_{n=0}^{\infty} \sum_{k=n}^{\infty}$.

Now equate Eqs. 2.40 and 2.42. Because the functions $r_{1}^{n} P_{n}^{m}\left(\cos \theta_{1}\right)$ are independent, we can equate all terms with same $n$ and $m$ to get:

$$
\frac{(n-m) !}{(n+m) !} \frac{1}{r_{2}^{n+1}} P_{n}^{m}\left(\cos \theta_{2}\right)=\sum_{k=n}^{\infty} 2 \frac{2 k+1}{R} \frac{(-)^{n+k+m}(k-m) !(k+n) !}{(k+m) !(k-n) ! n !(n+m) !} \frac{1}{R^{n}} Q_{k}^{m}\left(\bar{\xi}_{2}\right) P_{k}^{m}\left(\bar{\eta}_{2}\right),
$$

which can be simplified to obtain Eq. 2.28 ,

Eq. 2.27 can be derived in the same way as with Eq. 2.28- by plugging in Eq. 2.26 into the expansion of Green's function in terms of offset spherical harmonics, and using the independence of the Legendre functions.

\section{Comment on proofs}

These proofs may not be how we initially found the relationships. Here is one technique to find expansion coefficients using Matlab, with the example of Eq. 2.26. Create a length $n$ vector $\mathbf{v}$ of various $\theta$ values for $\left(\frac{r}{R}\right)^{n} P_{n}^{m}(\cos \theta)$, and create a $n$ by $n$ matrix $M$ of various $\theta$ and $k$ for $P_{k}^{m}(\bar{\xi}) P_{k}^{m}(\bar{\eta})$ and solving $M \mathbf{x}=\mathbf{v}$ for $\mathbf{x}$ (solving this is easy in Matlab). $\mathbf{x}$ contains numerical evaluations of the coefficients of the expansion, which hopefully can be used to guess the analytic form of the coefficients. This method can even be useful for infinite expansions if the series converges fast enough. 


\section{Chapter 3}

\section{Logopoles - A new class of solutions to Laplace's equation}

The logopoles are a new class of functions found by Eric Le Ru. Investigating these was the initial idea for this project, and this thesis is the first public available document that logopoles are mentioned. They have singularities on a finite line along the $z$ axis just like the spheroidal harmonics, so they can be used to describe solutions to physical problems outside objects. They were first introduced as a part of a fast converging solution to the problem of a point source near a sphere. In this chapter we first derive a number of equivalent definitions and properties of logopoles. The highlight of this chapter is possibly the new expression of spheroidal harmonics as a finite sum of spherical harmonics of the second kind (section 3.10). We only define logopoles with symmetry of revolution $(m=0)$ since they are not yet properly generalised to higher $m$.

Notation: Throughout this section we use the following shorthand notation:

$$
S_{n}=\left(\frac{R}{r}\right)^{n+1} P_{n}(\cos \theta) ; \quad \tilde{S}_{n}=(-)^{n+1}\left(\frac{r}{R}\right)^{n} Q_{n}(\cos \theta)
$$

Again $R$ is an arbitrary constant.

\subsection{Series definition}

The logopoles can be defined as an infinite series of multipoles:

$$
L_{n}=\sum_{k=0}^{\infty} \frac{n ! k !}{(n+k+1) !}\left(\frac{R}{r}\right)^{k+1} P_{k}(\cos \theta)=\sum_{k=0}^{\infty} \frac{n ! k !}{(n+k+1) !} S_{k}
$$

which converges only for $r>R$. As we will see later, logopoles can be analytically continued inside this radius.

This power series definition is straightforward but may not be convenient for practical calculations or manipulation. It is our first definition since it was the first appearance of the logopoles, in the solution of a point source near a sphere, so our derivations tend to use this definition frequently. This series may converge slowly as $r \rightarrow R$, but it seems to be numerically stable (see appendix on numerical instability).

\subsection{Expression in terms of offset multipoles of the second kind}

We will justify the following alternative expression for the logopoles:

$$
L_{n}=(-)^{n+1}\left(\frac{r^{\prime}}{R}\right)^{n} Q_{n}\left(\cos \theta^{\prime}\right)+\sum_{k=0}^{n}\left(\begin{array}{l}
n \\
k
\end{array}\right)(-)^{k}\left(\frac{r}{R}\right)^{k} Q_{k}(\cos \theta)=\tilde{S}_{n}^{\prime}-\sum_{k=0}^{n}\left(\begin{array}{l}
n \\
k
\end{array}\right) \tilde{S}_{k}
$$

The $Q_{n}(\cos \theta)$ have a logarithmic singularity on the entire $z$-axis which is why they are always discarded from standard solutions for physical problems. But in Eq. 3.2 the offset multipole singularity is partially cancelled by the singularities in the sum. We prove later that the singularity is only on the $z$-axis from 
O to O'. Despite the change in coordinate frame, this definition is more practical as it involves a finite sum and is valid everywhere except at the singularity, although it is not possible to numerically evaluate the logopoles along the $z$ axis using Eq. 3.2 because the individual terms are singular. Also, numerically computing logopoles at points far from the origin with this sum may produce errors due to subtracting terms that are very close in magnitude.

Justification of Eq. 3.2

Eq. 3.2 is actually a rearrangement of Eq. 3.3 below which can be viewed as a translation relation for the regular solid harmonics of the second kind:

$$
\tilde{S}_{n}^{\prime}=\sum_{p=0}^{n} \frac{n ! \tilde{S}_{p}}{p !(n-p) !}+\sum_{k=0}^{\infty} \frac{n ! k ! S_{k}}{(k+n+1) !} .
$$

This equation may not be known so we will justify it by induction on $n$. This is a justification because we only prove Eq. 3.3 up to some function of $\rho$, but it has been checked numerically that this function should be zero. First derive the base case starting from the known expansion for an offset point charge (Eq. 2.10 for $\mathrm{n}=0$ )

$$
S_{0}^{\prime}=\frac{R}{r^{\prime}}=\sum_{k=0}^{\infty} S_{k}
$$

We now integrate Eq. 3.4 with respect to $z$. The following integral relations will be useful. Eq. 3.5 is obtained by integrating Eq. 2.6, Eq. 3.7 is obtained by integrating Eq. 2.7. The $n=0$ case is treated separately.

$$
\begin{aligned}
& \int S_{n} \frac{\mathrm{d} z}{R}=-\frac{S_{n-1}}{n}+f(\rho) \quad(n>0) \\
& \int S_{0} \frac{\mathrm{d} z}{R}=\int \frac{\mathrm{d} z}{r}=-\ln \frac{r-z}{\rho}+f(\rho)=-\tilde{S}_{0}+f(\rho) \\
& \int \tilde{S}_{n} \frac{\mathrm{d} z}{R}=-\frac{\tilde{S}_{n+1}}{n+1}+f(\rho) .
\end{aligned}
$$

with $\rho=\sqrt{x^{2}+y^{2}}$ and $f(\rho)$ an arbitrary function. These also apply to multipoles in the primed frame because integrating with respect to $z$ is identical to integrating with respect to $z^{\prime}$. Integrating Eq. 3.4 . splitting off the first term in the sum and using Eqs. 3.5 and 3.6, then reindexing the sum, we obtain

$$
\tilde{S}_{0}^{\prime}=\tilde{S}_{0}+\sum_{k=0}^{\infty} \frac{S_{k}}{k+1}+f(\rho)
$$

It can be seen by plugging in explicit expressions and evaluating this expression at $z \rightarrow \infty$ that $f(\rho)=0$. Then we have shown the base case $n=0$ for Eq. 3.3.

Now begin the induction. Assume Eq. 3.3 is valid for $n$, and integrate it with respect to $z$ to obtain the $n+1^{\text {th }}$ case. We will use Eqs. $3.5-3.7$.

$$
\begin{aligned}
\int \tilde{S}_{n}^{\prime} \frac{\mathrm{d} z}{R} & =\sum_{p=0}^{n} \frac{n !}{p !(n-p) !} \int \tilde{S}_{p} \frac{\mathrm{d} z}{R}+\sum_{k=0}^{\infty} \frac{n ! k !}{(k+n+1) !} \int S_{k} \frac{\mathrm{d} z}{R} \\
=-\frac{\tilde{S}_{n+1}^{\prime}}{n+1} & =-\sum_{p=0}^{n} \frac{n !}{(p+1) !(n-p) !} \tilde{S}_{p+1}-\tilde{S}_{0}-\sum_{k=1}^{\infty} \frac{n !(k-1) !}{(k+n+1) !} S_{k-1}+f(\rho) \\
\Rightarrow \tilde{S}_{n+1}^{\prime} & =\sum_{p=-1}^{n} \frac{(n+1) !}{(p+1) !(n-p) !} \tilde{S}_{p+1}+\sum_{k=1}^{\infty} \frac{(n+1) !(k-1) !}{(k+n+1) !} S_{k-1}+f(\rho) \\
& =\sum_{p=0}^{n+1} \frac{(n+1) !}{p !(n+1-p) !} \tilde{S}_{p}+\sum_{k=0}^{\infty} \frac{(n+1) ! k !}{(k+n+2) !} S_{k}+f(\rho)
\end{aligned}
$$


which is case $n+1$ up to a function of $\rho$, so by induction Eq. 3.3 holds up to a function of $\rho$. It would be ideal to prove that $f(\rho)=0$. Naively it would seem that this problem of the arbitrary function could be avoided by applying definite integration between some reference point and the variable $z$. The reference point would be chosen to be where all the integrated functions are zero, but we could not find such a point. Another way to complete the proof would be to find some $z$ where Eq. 3.3 holds with no additional function of $\rho$. Obvious values of $z$ to try would be $\pm \infty$; here at least the sum over the irregular harmonics goes to zero. Showing that the rest then equals zero seems to be difficult; it may be helpful to use the alternative form of logopoles in the next section.

\subsection{Alternative form of logopoles}

Assuming Eq. 3.2 is correct, the logopoles can equivalently be expressed as

$$
\begin{aligned}
& L_{n}=\left(\frac{-r^{\prime}}{R}\right)^{n}\left[P_{n}\left(\cos \theta^{\prime}\right) L_{0}+W_{n-1}\left(\cos \theta^{\prime}\right)\right]-\sum_{k=0}^{n}\left(\begin{array}{l}
n \\
k
\end{array}\right)\left(\frac{-r}{R}\right)^{k} W_{k-1}(\cos \theta) \\
& \text { with } W_{n-1}=\sum_{k=1}^{n} \frac{1}{k} P_{n-k} P_{k-1} ; \quad W_{-1}=0
\end{aligned}
$$

which clearly distinguishes the logarithmic part $L_{0}$ from the polynomial part. Then it is clear that the logopoles are analytic everywhere except at a line of singularity from $\mathrm{O}$ to $\mathrm{O}$, i.e. for $\rho=0,0 \leq z \leq R$.

Derivation of Eq. 3.13

Begin with the expression in terms of multipoles of the second kind (Eq. 3.2) and insert the expression $Q_{n}=P_{n} Q_{0}-W_{n-1}$ :

$L_{n}=-\left(\frac{-r^{\prime}}{R}\right)^{n}\left[P_{n}\left(\cos \theta^{\prime}\right) Q_{0}\left(\cos \theta^{\prime}\right)-W_{n-1}\left(\cos \theta^{\prime}\right)\right]+\sum_{k=0}^{n}\left(\begin{array}{l}n \\ k\end{array}\right)\left(\frac{-r}{R}\right)^{k}\left[P_{k}(\cos \theta) Q_{0}(\cos \theta)-W_{k-1}(\cos \theta)\right]$

Applying the translation relation for regular spherical harmonics, Eq. 2.9.

$$
\begin{aligned}
L_{n}=-\left(\frac{-r^{\prime}}{R}\right)^{n}\left[P_{n}\left(\cos \theta^{\prime}\right) Q_{0}\left(\cos \theta^{\prime}\right)-W_{n-1}\left(\cos \theta^{\prime}\right)\right]+\left(\frac{-r^{\prime}}{R}\right)^{n} P_{n}\left(\cos \theta^{\prime}\right) Q_{0}(\cos \theta) & -\sum_{k=0}^{n}\left(\begin{array}{l}
n \\
k
\end{array}\right)\left(\frac{-r}{R}\right)^{k} W_{k-1}(\cos \theta)
\end{aligned}
$$

Then recognise $L_{0}=Q_{0}(\cos \theta)-Q_{0}\left(\cos \theta^{\prime}\right)$ to obtain Eq. 3.13 .

\subsection{Expression in terms of offset multipoles of the first kind}

Logopoles can also be written as a series of solid harmonics in the primed frame:

$$
L_{n}=\sum_{k=0}^{\infty} \frac{(-)^{k}}{n+k+1}\left(\frac{R}{r^{\prime}}\right)^{k+1} P_{k}\left(\cos \theta^{\prime}\right)=\sum_{k=0}^{\infty} \frac{(-)^{k}}{n+k+1} S_{k}^{\prime}
$$

This converges outside the sphere $r^{\prime}=R$. Like the first series definition of the logopoles this seems to be numerically stable, although the coefficients converge more slowly.

\section{Proof of Eq 3.16}

Begin with the series definition of $L_{n}$ in the non-primed frame $\mathrm{O}$ (Eq. 3.1). The solid harmonics centred at $\mathrm{O}$ can be expressed as a sum of solid harmonics centred at $\mathrm{O}$ ' by manipulating the translation relation for irregular solid harmonics (Eq. 2.10) - first translate the relation down the $z$ axis so that O' becomes $\mathrm{O}$ and $\mathrm{O}$ becomes $\mathrm{O}$ ", then replace $z$ with $-z$, and use the parity of the Legendre functions $P_{n}(-x)=(-)^{n} P_{n}(x)$. So we have

$$
S_{n}=\sum_{k=n}^{\infty}(-)^{n+k}\left(\begin{array}{l}
k \\
n
\end{array}\right) S_{k}^{\prime}
$$


Inserting this into Eq. 3.1

$$
L_{n}=\sum_{k=0}^{\infty} \frac{n ! k !}{(n+k+1) !} \sum_{p=k}^{\infty}(-)^{p+k} \frac{p !}{(p-k) ! k !} S_{p}^{\prime}
$$

Rearranging the order of summation:

$$
L_{n}=\sum_{p=0}^{\infty} \sum_{k=0}^{p} \frac{(-)^{k+p} n ! p !}{(n+k+1) !(p-k) !} S_{p}^{\prime} .
$$

Now we have to simplify the sum over $k$. Ignoring the $(-)^{p}$, the sum can be written using a binomial coefficient:

$$
\sum_{k=0}^{p} \frac{(-)^{k} n ! p !}{(n+k+1) !(p-k) !}=\frac{n ! p !}{(n+p+1) !} \sum_{k=0}^{p}(-)^{k}\left(\begin{array}{c}
n+p+1 \\
p-k
\end{array}\right)
$$

Now we can use Pascal's rule:

$$
\left(\begin{array}{c}
n+p+1 \\
p-k
\end{array}\right)=\left(\begin{array}{c}
n+p \\
p-k
\end{array}\right)+\left(\begin{array}{c}
n+p \\
p-k-1
\end{array}\right)
$$

Then we have (re-indexing $k \rightarrow k-1$ for the sum over the second binomial coefficient):

$$
\begin{aligned}
\sum_{k=0}^{p}(-)^{k}\left(\begin{array}{c}
n+p+1 \\
p-k
\end{array}\right) & =\sum_{k=0}^{p}(-)^{k}\left(\begin{array}{c}
n+p \\
p-k
\end{array}\right)-\sum_{k=1}^{p+1}(-)^{k}\left(\begin{array}{c}
n+p \\
p-k
\end{array}\right) \\
& =\sum_{k=0}^{p}(-)^{k}\left(\begin{array}{c}
n+p \\
p-k
\end{array}\right)-\sum_{k=0}^{p}(-)^{k}\left(\begin{array}{c}
n+p \\
p-k
\end{array}\right)+\left(\begin{array}{c}
n+p \\
p
\end{array}\right)
\end{aligned}
$$

In the last step the $k=0$ term in the second sum on the r.h.s. was extracted and we used the fact that the $k=p+1$ term is zero by the definition of binomial coefficients. The two sums cancel and we have the identity

$$
\sum_{k=0}^{p} \frac{(-)^{k} n ! p !}{(n+k+1) !(p-k) !}=\frac{1}{n+p+1}
$$

Insert this into Eq. 3.19 and relabel $p \rightarrow k$ to obtain Eq. 3.16 .

\subsection{Recurrence relation}

The logopoles obey the following recurrence relation:

$$
n L_{n}=-\frac{z^{\prime}}{R}(2 n-1) L_{n-1}-\left(\frac{r^{\prime}}{R}\right)^{2}(n-1) L_{n-2}+\frac{r}{R}
$$

This is only numerically stable near the singularity. For example at the point $\rho=2 R, z=0$, numerical errors become apparent (on the order of the magnitute of the function itself) at $n \approx 40$, while for $\rho=R / 2, z=0$, errors become apparent at $n \approx 240$.

\section{Derivation of Eq. 3.24;}

Begin with the series definition in terms of offset multipoles (Eq. 3.16). We use a rearrangement of the recurrence relation for irregular solid harmonics:

$$
\begin{aligned}
(k+2) S_{k+2} & =(2 k+3) \frac{R}{r} \cos \theta S_{k+1}-(k+1) \frac{R^{2}}{r^{2}} S_{k} \\
\Rightarrow S_{k} & =(2 k+3) \frac{z}{R} S_{k+1}-(k+2) \frac{r^{2}}{R^{2}} S_{k+2}
\end{aligned}
$$


Using this in the primed frame and inserting into Eq. 3.16 .

$$
\begin{aligned}
& n L_{n}=\sum_{k=0}^{\infty} \frac{(-)^{k} n}{n+k+1} \frac{1}{k+1}\left[\bar{z}^{\prime}(2 k+3) S_{k+1}^{\prime}-\bar{r}^{\prime}(k+2) S_{k+2}^{\prime}\right] \\
& \text { with shorthand notation } \bar{r}^{\prime}=\frac{r^{\prime}}{R} ; \quad \bar{z}^{\prime}=\frac{z^{\prime}}{R} .
\end{aligned}
$$

Now split into two sums, and re-index so that the spherical harmonics are order $k$ :

$$
n L_{n}=-\bar{z}^{\prime} \sum_{k=1}^{\infty} \frac{(-)^{k} n}{n+k} \frac{2 k+1}{k} S_{k}^{\prime}-\bar{r}^{\prime 2} \sum_{k=2}^{\infty} \frac{(-)^{k} n}{n+k-1} \frac{k}{k-1} S_{k}^{\prime}
$$

Now we want to extract $L_{n-1}$ and $L_{n-2}$, splitting off the desired prefactors:

$$
\begin{aligned}
& n \frac{2 k+1}{k}=2 n-1+\frac{n+k}{k} \\
& n \frac{k}{k-1}=n-1+\frac{n+k-1}{k-1}
\end{aligned}
$$

Applying Eq. 3.29 to the first sum in Eq. 3.28, and Eq. 3.30 to the second sum:

$$
\begin{aligned}
n L_{n}=-\bar{z}^{\prime}\left[(2 n-1) \sum_{k=1}^{\infty} \frac{(-)^{k}}{n+k} S_{k}^{\prime}+\sum_{k=1}^{\infty} \frac{(-)^{k}}{k} S_{k}^{\prime}\right]-\bar{r}^{2}\left[(n-1) \sum_{k=2}^{\infty} \frac{(-)^{k}}{n+k-1} S_{k}^{\prime}+\sum_{k=2}^{\infty} \frac{(-)^{k}}{k-1} S_{k}^{\prime}\right] \\
=-(2 n-1) \bar{z}^{\prime} L_{n-1}+\frac{2 n-1}{n} \bar{z}^{\prime} S_{0}^{\prime}-\bar{z}^{\prime} \sum_{k=1}^{\infty} \frac{(-)^{k}}{k} S_{k}^{\prime} \\
\quad-(n-1) \bar{r}^{\prime 2} L_{n-2}+\bar{r}^{\prime 2}\left[S_{0}^{\prime}-\frac{n-1}{n} S_{1}^{\prime}\right]-\bar{r}^{\prime 2} \sum_{k=2}^{\infty} \frac{(-)^{k}}{k-1} S_{k}^{\prime}
\end{aligned}
$$

The first two terms on the r.h.s. of Eq. 3.24 have been revealed, now we have to show that the rest of Eq. 3.31 equates to $r / R$. Note for the lowest orders:

$$
\bar{z}^{\prime} S_{0}^{\prime}=\bar{r}^{\prime 2} S_{1}^{\prime}=\cos \theta^{\prime} ; \quad \bar{r}^{\prime 2} S_{0}^{\prime}=\bar{r}^{\prime}
$$

so that (and re-indexing the last sum of Eq. 3.31):

$$
\begin{aligned}
n L_{n}+(2 n-1) \bar{z}^{\prime} L_{n-1}+(n-1) \bar{r}^{\prime 2} L_{n-2} & =\cos \theta^{\prime}+\bar{r}^{\prime}-\sum_{k=1}^{\infty} \frac{(-)^{k}}{k}\left(\bar{z}^{\prime} S_{k}^{\prime}-\bar{r}^{\prime 2} S_{k+1}^{\prime}\right) \\
& =\cos \theta^{\prime}+\bar{r}^{\prime}-\sum_{k=1}^{\infty}(-)^{k}\left(\bar{r}^{\prime 2} S_{k+1}^{\prime}-2 \bar{z}^{\prime} S_{k}^{\prime}-S_{k-1}^{\prime}\right)
\end{aligned}
$$

The last step used a rearrangement of the recurrence relation for $S_{k}^{\prime}$. Then re-indexing sums again and adding the lowest orders to the sums so that they can be extended down to $k=0$ :

$n L_{n}+(2 n-1) \bar{z}^{\prime} L_{n-1}+(n-1) \bar{r}^{2} L_{n-2}=\cos \theta^{\prime}+\bar{r}^{\prime}+\left(\bar{r}^{2}+2 \bar{z}+1\right) \sum_{k=0}^{\infty}(-)^{k} S_{k}^{\prime}-2 \bar{z}^{\prime} S_{0}^{\prime}-\bar{r}^{2}\left(S_{0}^{\prime}-S_{1}^{\prime}\right)$

Then use the following identities (the first is obtained by reflecting the translation relation for irregular spherical harmonics Eq. 2.10 about $z=0$ and using the parity of $P_{n}$ ):

$$
\sum_{k=0}^{\infty}(-)^{k} S_{k}^{\prime}=\frac{R}{r} ; \quad \bar{r}^{2}+2 \bar{z}^{\prime}+1=\frac{r^{2}}{R^{2}}
$$

and cancel the remaining terms in the r.h.s. of Eq. 3.34 to find that it does equal $r / R$. 


\subsection{Lowest orders of logopoles}

There are a range of expressions for $L_{0}$. First from Eq. 3.2 .

$$
L_{0}=\tilde{S}_{0}^{\prime}-\tilde{S}_{0}=Q_{0}(\cos \theta)-Q_{0}\left(\cos \theta^{\prime}\right)=2 Q_{0}(\bar{\xi}) P_{0}(\bar{\eta})
$$

The last equality is derived in section 3.8 . Note the following expressions for $Q_{0}$ :

$$
Q_{0}(\cos \theta)=\frac{1}{2} \log \frac{1+\cos \theta}{1-\cos \theta}=\frac{1}{2} \ln \frac{r+z}{r-z}=\ln \frac{\rho}{r-z}=\ln \frac{r+z}{\rho} .
$$

The lowest orders of logopoles are best derived from the recurrence relation or Eq. 3.13, and are (with $u^{\prime}=\cos \theta^{\prime}$ and $\left.\bar{r}^{\prime}=r^{\prime} / R\right)$ :

$$
\begin{aligned}
L_{0}= & \ln \frac{r^{\prime}-z^{\prime}}{r-z}=\ln \frac{r+z}{r^{\prime}+z^{\prime}} \\
L_{1}= & -\left[u^{\prime} L_{0}+1\right] \bar{r}^{\prime}+\bar{r} \\
L_{2}= & \frac{1}{2}\left[\left(3 u^{\prime 2}-1\right) L_{0}+3 u^{\prime}\right] \bar{r}^{\prime 2}+\frac{1}{2}\left[-3 u^{\prime} \bar{r}^{\prime}+1\right] \bar{r} \\
L_{3}= & -\frac{1}{2}\left[\left(5 u^{\prime 3}-3 u^{\prime}\right) L_{0}+5 u^{\prime 2}-\frac{4}{3}\right] \bar{r}^{\prime 3}+\frac{1}{2}\left[5 u^{\prime 2} \bar{r}^{\prime 2}-\frac{4}{3} \bar{r}^{\prime 2}-\frac{5}{3} u^{\prime} \bar{r}^{\prime}+\frac{2}{3}\right] \bar{r} \\
L_{4}= & \frac{1}{8}\left[\left(35 u^{\prime 4}-30 u^{\prime 2}+3\right) L_{0}+35 u^{\prime 3}-\frac{55}{3} u^{\prime}\right] \bar{r}^{\prime 4}+\frac{1}{8}\left[-35 u^{\prime 3} \bar{r}^{\prime 3}+\frac{55}{3} u^{\prime} \bar{r}^{\prime 3}+\frac{35}{3} u^{\prime 2} \bar{r}^{\prime 2}-3 \bar{r}^{\prime 2}-\frac{14}{3} u^{\prime} \bar{r}^{\prime}+2\right] \bar{r} \\
L_{5}= & -\frac{1}{8}\left[\left(63 u^{\prime 5}-70 u^{\prime 3}+15 u^{\prime}\right) L_{0}+\left(63 u^{\prime 4}-49 u^{\prime 2}+\frac{64}{15}\right)\left(1-\frac{r}{r^{\prime}}\right)\right] \bar{r}^{\prime 5} \\
& +\frac{1}{8}\left[-21 u^{\prime 3} \bar{r}^{\prime 3}+\frac{161}{15} u^{\prime} \bar{r}^{\prime 3}+\frac{42}{5} u^{\prime 2} \bar{r}^{\prime 2}-\frac{32}{15} \bar{r}^{\prime 2}-\frac{18}{5} u^{\prime} \bar{r}^{\prime}+\frac{8}{5}\right] \bar{r}
\end{aligned}
$$

\subsection{Differentiation and integration along $z$ axis}

The logopoles obey the following relations:

$$
\begin{aligned}
& R \frac{\partial}{\partial z} L_{n}=-n L_{n-1}+\frac{R}{r} \\
& \int L_{n} \frac{\mathrm{d} z}{R}=\frac{-1}{n+1}\left(L_{n+1}+\ln \frac{r-z}{\rho}\right)+f(\rho)
\end{aligned}
$$

where $f(\rho)$ is an arbitrary function of $\rho$.

Proof of Eq. 3.35

Start from the series definition of $L_{n}$ (Eq. 3.1)

$$
R \frac{\partial}{\partial z} L_{n}=\sum_{k=0}^{\infty} \frac{n ! k !}{(n+k+1) !} R \frac{\partial}{\partial z} S_{k}
$$

and apply Eq. 2.6. $R \partial_{z} S_{n}=-(n+1) S_{n+1}$ :

$$
\begin{aligned}
R \frac{\partial}{\partial z} L_{n} & =-\sum_{k=1}^{\infty} \frac{n ! k !}{(n+k+1) !}(k+1) S_{k+1} \\
& =-\sum_{k=0}^{\infty} \frac{n ! k !}{(n+k) !} S_{k} \quad(k+1 \rightarrow k) \\
& =-n L_{n-1}+\frac{R}{r} .
\end{aligned}
$$

And Eq. 3.36 comes directly from integrating Eq. 3.35 with respect to $z$. 


\subsection{Link to spheroidal harmonics}

Logopoles can also be written as a finite sum of barred spheroidal harmonics and vice versa:

$$
\begin{array}{r}
Q_{n}(\bar{\xi}) P_{n}(\bar{\eta})=\sum_{k=0}^{n} \frac{(-)^{k}}{2} \frac{(n+k) !}{k !^{2}(n-k) !} L_{k} \\
L_{n}=\sum_{k=0}^{n} \frac{2(-)^{k}(2 k+1) n !^{2}}{(n-k) !(n+k+1) !} Q_{k}(\bar{\xi}) P_{k}(\bar{\eta})
\end{array}
$$

Eq. 3.38 is numerically unstable in all space for large $n$; Eq. 3.39 is much more accurate and only shows inaccuracy either very close or very far from the singularity.

Proof of Eq. 3.38

Compare the expansions of $L_{n}$ and $Q_{n}(\bar{\xi}) P_{n}(\bar{\eta})$ in terms of spherical harmonics:

$$
\begin{aligned}
Q_{n}(\bar{\xi}) P_{n}(\bar{\eta}) & =\sum_{k=n}^{\infty} \frac{k !^{2}}{2(k-n) !(k+n+1) !} S_{k}, \\
L_{n} & =\sum_{k=0}^{\infty} \frac{n ! k !}{(n+k+1) !} S_{k} .
\end{aligned}
$$

The sum in $Q_{n}(\bar{\xi}) P_{n}(\bar{\eta})$ starts from $k=n$, but it can be written as

$$
Q_{n}(\bar{\xi}) P_{n}(\bar{\eta})=\sum_{k=0}^{\infty} \frac{k ! k(k-1) \ldots(k-n+1)}{2(k+n+1) !} S_{k}
$$

so that the sum extends to $k=0$. This is identical to Eq. 3.40 since all terms with $k<n$ are zero anyway. Now assume that the spheroidal harmonics can be written as a finite sum of logopoles:

$$
Q_{n}(\bar{\xi}) P_{n}(\bar{\eta})=\sum_{p=0}^{n} \alpha_{p}^{n} L_{p}
$$

Insert the spherical harmonic expansions of $L_{n}$ and $Q_{n}(\bar{\xi}) P_{n}(\bar{\eta})$ into Eq. 3.42. Because $S_{k}$ are linearly independent, the coefficients $\alpha_{p}^{n}$ must satisfy:

$$
\frac{k(k-1) \ldots(k-n+1)}{(n+k+1) !}=\sum_{p=0}^{n} \frac{2 p !}{(p+k+1) !} \alpha_{p}^{n}
$$

We want to use the fact that any degree $n$ polynomial $p(t)$ of an integer argument $t$ can be expressed as a sum of binomial coefficients:

$$
\begin{gathered}
p(t)=\sum_{q=0}^{n} a_{q}\left(\begin{array}{l}
t \\
q
\end{array}\right) \\
\text { with } \quad a_{q}=\sum_{i=0}^{q}(-)^{q+i}\left(\begin{array}{l}
q \\
i
\end{array}\right) p(i)
\end{gathered}
$$

This can be proved by substituting $p(i)=\sum_{k=0}^{n}\left(\begin{array}{l}i \\ k\end{array}\right)$ into the expression for $a_{q}$, rearranging the order of summation, and using the identity $\sum_{i=0}^{q}(-)^{q+i}\left(\begin{array}{l}q \\ i\end{array}\right)\left(\begin{array}{l}i \\ k\end{array}\right)=\delta_{q k}[15$. 
So we rearrange Eq. 3.43 to express it using binomial coefficients:

$$
\begin{aligned}
k(k-1) \ldots(k-n+1) & =\sum_{p=0}^{n} 2 \frac{p !(n+k+1) !}{(p+k+1) !} \alpha_{p}^{n} \\
& =\sum_{p=0}^{n} 2\left(\begin{array}{c}
k+n+1 \\
n-p
\end{array}\right) p !(n-p) ! \alpha_{p}^{n} \\
& =\sum_{q=0}^{n} 2\left(\begin{array}{c}
k+n+1 \\
q
\end{array}\right) q !(n-q) ! \alpha_{n-q}^{n} \quad q \equiv n-p
\end{aligned}
$$

In terms of Eq. 3.44, we have $t=n+k+1, p(t)=(t-n-1)(t-n-2) \ldots(t-2 n)$, so

$$
a_{q}=2 q !(n-q) ! \alpha_{n-q}^{n}=\sum_{i=0}^{q}(-)^{q+i}\left(\begin{array}{c}
q \\
i
\end{array}\right)(i-n-1)(i-n-2) \ldots(i-2 n) .
$$

Since $i<n$, we can write

$$
p(i)=(-)^{n} \frac{(2 n-i) !}{(n-i) !}=(-)^{n} n !\left(\begin{array}{c}
2 n-i \\
n
\end{array}\right)
$$

Then

$$
\begin{aligned}
\alpha_{n-q}^{n} & =\frac{1}{2}\left(\begin{array}{c}
n \\
q
\end{array}\right) \sum_{i=0}^{q}(-)^{n+q+i}\left(\begin{array}{c}
q \\
i
\end{array}\right)\left(\begin{array}{c}
2 n-i \\
n
\end{array}\right) \\
& =\frac{1}{2}\left(\begin{array}{l}
n \\
p
\end{array}\right) \sum_{i=0}^{n-p}(-)^{p+i}\left(\begin{array}{c}
n-p \\
i
\end{array}\right)\left(\begin{array}{c}
2 n-i \\
n
\end{array}\right)
\end{aligned}
$$

This expression can be simplified using the identity ([18], pg 619, or [2], pg.1394):

$$
\sum_{j=0}^{m}(-)^{j}\left(\begin{array}{c}
m \\
j
\end{array}\right)\left(\begin{array}{c}
a+j \\
n
\end{array}\right)=(-)^{m}\left(\begin{array}{c}
a \\
n-m
\end{array}\right)
$$

making substitutions $m \rightarrow n-p, j \rightarrow n-p-i, a=n+p$ and using $\left(\begin{array}{c}n-p \\ n-p-i\end{array}\right)=\left(\begin{array}{c}n-p \\ i\end{array}\right)$ :

$$
\begin{aligned}
& \sum_{i=0}^{n-p}(-)^{p+i}\left(\begin{array}{c}
n-p \\
i
\end{array}\right)\left(\begin{array}{c}
2 n-i \\
n
\end{array}\right)=(-)^{p}\left(\begin{array}{c}
n+p \\
p
\end{array}\right) \\
\Rightarrow \alpha_{p}^{n}= & \frac{(-)^{p}}{2}\left(\begin{array}{c}
n \\
p
\end{array}\right)\left(\begin{array}{c}
n+p \\
p
\end{array}\right)=\frac{(-)^{p}}{2} \frac{(n+p) !}{(n-p) ! p !^{2}}
\end{aligned}
$$

The first few orders are

$$
\begin{aligned}
Q_{0}(\bar{\xi}) P_{0}(\bar{\eta}) & =\frac{L_{0}}{2} \\
Q_{1}(\bar{\xi}) P_{1}(\bar{\eta}) & =\frac{L_{0}}{2}-L_{1} \\
Q_{2}(\bar{\xi}) P_{2}(\bar{\eta}) & =\frac{L_{0}}{2}-3 L_{1}+3 L_{2} \\
Q_{3}(\bar{\xi}) P_{3}(\bar{\eta}) & =\frac{L_{0}}{2}-6 L_{1}+15 L_{2}-10 L_{3}
\end{aligned}
$$

Note that the expansion coefficients of $Q_{n}(\bar{\xi}) P_{n}(\bar{\eta})$ in terms of $L_{k}$ are almost identical to that of the coefficients for $P_{n}(\bar{\xi}) P_{n}(\bar{\eta})$ in terms of $r^{k} P_{k}(\cos \theta)$ (Eq. 2.25 for $m=0$ ). They differ only by $2(-)^{k}$. By noting that Eq. 2.25 can be inverted to expand $r^{n} P_{n}(\cos \theta)$ in terms of $P_{k}(\bar{\xi}) P_{k}(\bar{\eta})$ (Eq. 2.26), we can deduce the expansion of $L_{n}$ in terms of $Q_{k}(\bar{\xi}) P_{k}(\bar{\eta})$. 
We now derive a key identity that makes Eq. 2.26 the inverse of Eq. 2.25. Inserting Eq. 2.25 into Eq. 2.26 ,

$$
\begin{aligned}
\left(\frac{r}{R}\right)^{n} P_{n}(\cos \theta) & =\sum_{k=0}^{n} \frac{(2 k+1) n !^{2}}{(n-k) !(n+k+1) !} \sum_{p=0}^{k} \frac{(-)^{k+p}(k+p) !}{p !^{2}(k-p) !}\left(\frac{r}{R}\right)^{p} P_{p}(\cos \theta) \\
& =\sum_{p=0}^{n} \sum_{k=p}^{n} \frac{(2 k+1) n !^{2}}{(n-k) !(n+k+1) !} \frac{(-)^{k+p}(k+p) !}{p !^{2}(k-p) !}\left(\frac{r}{R}\right)^{p} P_{p}(\cos \theta)
\end{aligned}
$$

It must be true that

$$
\sum_{k=p}^{n} \frac{(2 k+1) n !^{2}}{(n-k) !(n+k+1) !} \frac{(-)^{k+p}(k+p) !}{p !^{2}(k-p) !}=\delta_{n p}
$$

Now do the same with logopoles - first assume an expansion with coefficients $\beta_{n}^{k}: L_{n}=\sum_{k=0}^{n} \beta_{n}^{k} Q_{n}(\bar{\xi}) P_{n}(\bar{\eta})$ and insert Eq. 3.38 .

$$
L_{n}=\sum_{k=0}^{n} \beta_{n}^{k} \sum_{p=0}^{k} \frac{(-)^{p}}{2} \frac{(k+p) !}{p !^{2}(k-p) !} L_{p}
$$

Again it must be true that

$$
\sum_{k=p}^{n} \beta_{n}^{k} \frac{(-)^{p}}{2} \frac{(k+p) !}{p !^{2}(k-p) !}=\delta_{n p}
$$

By comparing this to Eq. 3.46, we must have

$$
\beta_{n}^{k}=\frac{2(-)^{k}(2 k+1) n !^{2}}{(n-k) !(n+k+1) !}
$$

which are the coefficients of Eq. 3.39

\subsection{Integral form}

The logopoles can be expressed as the potential created by a line charge with density $q(z)=(1-z / R)^{n}$ :

$$
L_{n}=\int_{0}^{R}\left(1-\frac{\tilde{z}}{R}\right)^{n} \frac{\mathrm{d} \tilde{z}}{\sqrt{\rho^{2}+(z-\tilde{z})^{2}}}=\int_{0}^{1} \frac{(1-u)^{n} \mathrm{~d} u}{\sqrt{\rho^{2}+(z-R u)^{2}}}
$$

The line charge distribution is $q=(1-u)^{n}$ which ranges from $q=1$ at $u=0$, to $q=0$ at $u=1$ (for $n>0) . q$ is positive which means that the logopoles are positive everywhere in space. The potential of any continuous finite line charge can be expanded in terms of logopoles by expanding the charge distribution as a power series in $(1-u)$.

Derivation of Eq. 3.50

The spheroidal harmonics can be represented as the potential of a line charge distribution on the segment from $z=-R$ to $R$ [16]:

$$
Q_{n}(\xi) P_{n}(\eta)=\frac{R}{2} \int_{-1}^{1} \frac{P_{n}(u) \mathrm{d} u}{\sqrt{\rho^{2}+(z-R u)^{2}}}
$$

We actually need the corresponding equation for barred spheroidal harmonics with singularities from $z=0$ to $R$, which can be obtained by coordinate changing to barred coordinates (see section ??). First express this integral completely in terms of $\xi$ and $\eta$, plugging in $z=R \xi \eta$ and $\rho=R \sqrt{\left(\xi^{2}-1\right)\left(1-\eta^{2}\right)}$.

$$
Q_{n}(\xi) P_{n}(\eta)=\frac{R}{2} \int_{-1}^{1} \frac{P_{n}(u) \mathrm{d} u}{\sqrt{R^{2}\left(\xi^{2}-1\right)\left(1-\eta^{2}\right)+(R \xi \eta-R u)^{2}}}
$$


Then change $\xi \rightarrow \bar{\xi}$ and $\eta \rightarrow \bar{\eta}$. The relationship will still hold because this is only a relabelling. Then plug in the expressions of $\bar{\xi}$ and $\bar{\eta}$ in terms of $z$ and $\rho: R \sqrt{\left(\bar{\xi}^{2}-1\right)\left(1-\bar{\eta}^{2}\right)}=2 \rho$ and $R \bar{\xi} \bar{\eta}=2 z-R$. Also change the dummy variable $u \rightarrow 2 u-1$. After simplifications:

$$
Q_{n}(\bar{\xi}) P_{n}(\bar{\eta})=\frac{R}{2} \int_{0}^{1} \frac{P_{n}(2 u-1) \mathrm{d} u}{\sqrt{\rho^{2}+(z-R u)^{2}}}
$$

Since the logopoles can be written as a finite sum of offset spheroidal harmonics, we can find the line charge distribution that creates logopole potentials. Inserting Eq. 3.53 into the expansion of logopoles in terms of barred spheroidal harmonics, Eq. 3.39 .

$$
\begin{aligned}
L_{n} & =\sum_{k=0}^{n} \frac{2(-)^{k}(2 k+1) n !^{2}}{(n-k) !(n+k+1) !} \frac{R}{2} \int_{0}^{1} \frac{P_{k}(2 u-1) \mathrm{d} u}{\sqrt{\rho^{2}+(z-R u)^{2}}} \\
& =\int_{0}^{1} \frac{R \mathrm{~d} u}{\sqrt{\rho^{2}+(z-R u)^{2}}} \sum_{k=0}^{n} \frac{(-)^{k}(2 k+1) n !^{2}}{(n-k) !(n+k+1) !} P_{k}(2 u-1)
\end{aligned}
$$

Now we present an identity to simplify the sum. Evaluate the expansion of $r^{n} P_{n}(\cos \theta)$ in terms of $P_{k}(\bar{\xi}) P_{k}(\bar{\eta})$ (Eq. 2.26) with $m=0, \bar{\eta}=\cos \theta=-1$, (which means $\left.r=(\bar{\xi}-1) R / 2\right)$ :

$$
\sum_{k=0}^{n} \frac{(-)^{k}(2 k+1) n !^{2}}{(n-k) !(n+k+1) !} P_{k}(\bar{\xi})=(-)^{n}\left(\frac{\bar{\xi}-1}{2}\right)^{n}
$$

Insert this into Eq. 3.54 with $\bar{\xi} \rightarrow 2 u-1=2 \tilde{z} / R-1$ to obtain Eq. 3.50 .

\subsection{Alternate expression for $Q_{n}(\xi) P_{n}(\eta)$}

We derive a new formula expressing the relatively common irregular spheroidal harmonics as a finite sum of the rarely used offset spherical harmonics of the second kind:

$$
Q_{n}(\xi) P_{n}(\eta)=\frac{1}{2} \sum_{k=0}^{n}\left(\begin{array}{c}
n \\
k
\end{array}\right)\left(\begin{array}{c}
n+k \\
k
\end{array}\right)\left[(-)^{k} \tilde{S}_{k}^{\prime}-(-)^{n} \tilde{S}_{k}^{\prime \prime}\right]
$$

Note in this section we use $\tilde{S}_{k}=(-)^{k+1}\left(\frac{r}{2 R}\right)^{k} Q_{k}(\cos \theta)$ (with $2 R$ instead of $R$ before). Note Eq. 3.56 has not been strictly proven since the derivation relies on Eq. 3.2 which is not strictly proven. But Eq. 3.56 has been checked in Matlab to be correct, although it is numerically unstable in regions of space where $\tilde{S}_{k}^{\prime}$ and $\tilde{S}_{k}^{\prime \prime}$ are close in magnitude. A similar expression can be written for the barred spheroidal harmonics using the coordinate frames O and O' instead of O" and O'.

Derivation of Eq. 3.56

First note that we will work with stretched logopoles $\hat{L}_{n}$ which have their singularity from $z=-R$ to $R$. The scaling/translation transformation between $\hat{L}_{n}$ and $L_{n}$ is the same as that for $Q_{n}(\xi) P_{n}(\eta)$ and $Q_{n}(\bar{\xi}) P_{n}(\bar{\eta})$. By coordinate changing Eq. 3.38 we have:

$$
Q_{n}(\xi) P_{n}(\eta)=\frac{1}{2} \sum_{k=0}^{n}(-)^{k}\left(\begin{array}{l}
n \\
k
\end{array}\right)\left(\begin{array}{c}
n+k \\
k
\end{array}\right) \hat{L}_{k}
$$

And by coordinate changing Eq. 3.2 we have:

$$
\hat{L}_{n}=\tilde{S}_{k}^{\prime}-\sum_{p=0}^{k}\left(\begin{array}{l}
k \\
p
\end{array}\right) \tilde{S}_{p}^{\prime \prime}
$$


Again these $\tilde{S}_{n}$ have $2 R$ instead of $R$. Combining these two expansions:

$$
\begin{aligned}
Q_{n}(\xi) P_{n}(\eta) & =\frac{1}{2} \sum_{k=0}^{n}(-)^{k}\left(\begin{array}{c}
n \\
k
\end{array}\right)\left(\begin{array}{c}
n+k \\
k
\end{array}\right)\left[\tilde{S}_{k}^{\prime}-\sum_{p=0}^{k}\left(\begin{array}{c}
k \\
p
\end{array}\right) \tilde{S}_{p}^{\prime \prime}\right] \\
& =\frac{1}{2} \sum_{k=0}^{n}(-)^{k}\left(\begin{array}{c}
n \\
k
\end{array}\right)\left(\begin{array}{c}
n+k \\
k
\end{array}\right) \tilde{S}_{k}^{\prime}-\frac{1}{2} \sum_{p=0}^{n} \sum_{k=p}^{n}(-)^{k}\left(\begin{array}{c}
n \\
k
\end{array}\right)\left(\begin{array}{c}
n+k \\
k
\end{array}\right)\left(\begin{array}{c}
k \\
p
\end{array}\right) \tilde{S}_{p}^{\prime \prime}
\end{aligned}
$$

The last sum over $k$ can be simplified. Now we will derive a binomial identity for this purpose. $P_{n}(\xi) P_{n}(\eta)$ can be expanded in terms of $\hat{S}_{k}^{\prime}$ or $\hat{S}_{k}^{\prime \prime}$. We equate these expansions and expand $\hat{S}_{k}^{\prime}$ in terms of $\hat{S}_{k}^{\prime \prime}$ using Eq. 2.9, then rearrange the summation order:

$$
\begin{aligned}
P_{n}(\xi) P_{n}(\eta)=\sum_{k=0}^{n}(-)^{n+k}\left(\begin{array}{c}
n \\
k
\end{array}\right)\left(\begin{array}{c}
n+k \\
k
\end{array}\right) \hat{S}_{k}^{\prime \prime} & =\sum_{q=0}^{n}\left(\begin{array}{c}
n \\
q
\end{array}\right)\left(\begin{array}{c}
n+q \\
q
\end{array}\right) \hat{S}_{q}^{\prime} \\
& =\sum_{q=0}^{n}\left(\begin{array}{c}
n \\
q
\end{array}\right)\left(\begin{array}{c}
n+q \\
q
\end{array}\right) \sum_{p=0}^{q}(-)^{q+p}\left(\begin{array}{c}
q \\
p
\end{array}\right) \hat{S}_{p}^{\prime \prime} \\
& =\sum_{p=0}^{n} \sum_{q=p}^{n}(-)^{q+p}\left(\begin{array}{c}
n \\
q
\end{array}\right)\left(\begin{array}{c}
n+q \\
q
\end{array}\right)\left(\begin{array}{c}
q \\
p
\end{array}\right) \hat{S}_{p}^{\prime \prime}
\end{aligned}
$$

and by the orthogonality of the spherical harmonics, each coefficient of $S_{k}$ on the left hand side must be equal to the coefficient of $S_{p}$ on the right hand side when $p=k$, so

$$
\sum_{q=p}^{n}(-)^{q}\left(\begin{array}{l}
n \\
q
\end{array}\right)\left(\begin{array}{c}
n+q \\
q
\end{array}\right)\left(\begin{array}{l}
q \\
p
\end{array}\right)=(-)^{n}\left(\begin{array}{c}
n \\
p
\end{array}\right)\left(\begin{array}{c}
n+p \\
p
\end{array}\right)
$$

Apply this identity to Eq. 3.60 to obtain Eq. 3.56 .

\subsection{Attempt at expanding solid harmonics with logopoles}

The irregular solid spherical harmonics cannot be expanded in terms of logopoles; an attempt can be made by starting with the expansion of solid harmonics in terms of $Q_{n}(\bar{\xi}) P_{n}(\bar{\eta})$ (Eq. 2.28) and applying the expansion of spheroidal harmonics in terms of logopoles (Eq. 3.38), then rearranging the order of summation. For $S_{0}$ :

$$
\begin{aligned}
S_{0}=\frac{R}{r} & =\sum_{n=0}^{\infty} 2(2 n+1)(-)^{n} Q_{n}(\bar{\xi}) P_{n}(\bar{\eta}) \\
& =\sum_{n=0}^{\infty}(2 n+1)(-)^{n} \sum_{k=0}^{n}(-)^{k} \frac{(n+k) !}{k !^{2}(n-k) !} L_{k} \\
& =\sum_{k=0}^{\infty} \sum_{n=k}^{\infty}(2 n+1)(-)^{n+k} \frac{(n+k) !}{k !^{2}(n-k) !} L_{k}
\end{aligned}
$$

But the coefficient of $L_{0}$ in this expansion is $\sum_{n=0}^{\infty}(2 n+1)(-)^{n}$ which diverges. This also suggests that Green's function cannot be expanded in terms of logopoles, since $S_{0}$ is a special case of Green's function where the point source is at the origin. 


\subsection{Symmetric and antisymmetric logopoles}

The logopoles have no parity about $z$, but for potential applications to problems with reflective symmetry, we investigate defining anti/symmetric logopoles.

\subsubsection{Definitions}

We introduce symmetric logopoles $L_{n}^{s}$ as:

$$
\begin{aligned}
L_{n}^{s} & =\frac{1}{2}\left[L_{n}(x, y, z)+L_{n}(x, y,-z)\right] \\
& =\sum_{k=0}^{\infty} \frac{n ! k !}{(n+k+1) !}\left(\frac{R}{r}\right)^{k+1}\left[P_{k}(\cos \theta)-P_{k}(-\cos \theta)\right] \\
& =\sum_{\substack{k=0 \\
k \text { even }}}^{\infty} \frac{n ! k !}{(n+k+1) !}\left(\frac{R}{r}\right)^{k+1} P_{k}(\cos \theta)
\end{aligned}
$$

The last identity comes from the parity of the Legendre polynomials $P_{k}(-x)=(-)^{k} P_{k}(x)$. And the antisymmetric logopoles:

$$
\begin{aligned}
L_{n}^{a} & =\frac{1}{2}\left[L_{n}(x, y, z)-L_{n}(x, y,-z)\right] \\
& =\sum_{\substack{k=1 \\
k \text { odd }}}^{\infty} \frac{n ! k !}{(n+k+1) !}\left(\frac{R}{r}\right)^{k+1} P_{k}(\cos \theta)
\end{aligned}
$$

Note that $L_{n}=L_{n}^{s}+L_{n}^{a}$. Using the expression for $L_{n}$ in terms of offset multipoles, the symmetric and antisymmetric logopoles can be written as:

$$
\begin{aligned}
L_{n}^{s} & =\tilde{S}_{n}^{\prime}-(-)^{n} \tilde{S}_{n}^{\prime \prime}-\sum_{k=0}^{n}\left(\begin{array}{l}
n \\
k
\end{array}\right)\left(1-(-)^{k}\right) \tilde{S}_{k} \\
& =\tilde{S}_{n}^{\prime}-(-)^{n} \tilde{S}_{n}^{\prime \prime}-2 \sum_{k \text { odd }}^{n}\left(\begin{array}{l}
n \\
k
\end{array}\right) \tilde{S}_{k} \\
L_{n}^{a} & =\tilde{S}_{n}^{\prime}+(-)^{n} \tilde{S}_{n}^{\prime \prime}-2 \sum_{k \text { even }}^{n}\left(\begin{array}{l}
n \\
k
\end{array}\right) \tilde{S}_{k}
\end{aligned}
$$

The first few orders are

$$
\begin{aligned}
L_{0}^{s} & =\frac{1}{2}\left(\tilde{S}_{0}^{\prime}-\tilde{S}_{0}^{\prime \prime}\right)=\frac{1}{2} \ln \frac{r^{\prime}-z^{\prime}}{r^{\prime \prime}-z^{\prime \prime}}=\frac{1}{2} \ln \frac{r^{\prime \prime}+z^{\prime \prime}}{r^{\prime}+z^{\prime}} \\
L_{0}^{a} & =\frac{1}{2} \ln \frac{\left(r^{\prime}-z^{\prime}\right)\left(r^{\prime \prime}-z^{\prime \prime}\right)}{(r-z)^{2}}=\frac{1}{2} \ln \frac{(r+z)^{2}}{\left(r^{\prime}-z^{\prime}\right)\left(r^{\prime \prime}-z^{\prime \prime}\right)} \\
L_{1}^{s} & =\frac{1}{2}\left(\tilde{S}_{1}^{\prime}+\tilde{S}_{1}^{\prime \prime}\right)-\tilde{S}_{1}=L_{0}^{s}-\bar{z} L_{0}^{a}+r-\frac{r^{\prime}+r^{\prime \prime}}{2 R} \\
L_{1}^{a} & =L_{0}^{a}-\bar{z} L_{0}^{s}+\frac{r^{\prime \prime}-r^{\prime}}{2 R} .
\end{aligned}
$$

The antisymmetric logopoles are proportional to the potential created by a line source between O" and O' with line charge density $(1-z / R)^{n}$ (except for $L_{0}^{a}$ which has density $z /|z|$ ). For the symmetric logopoles, the line charge density is $|1-z / R|^{n}$.

\subsubsection{Spheroidal harmonics as a sum of anti/symmetric logopoles}

The spheroidal harmonics (centred at the origin) can be written as a sum of either symmetric or antisymmetric logopoles:

$$
\left.Q_{n}(\xi) P_{n}(\eta)=\sum_{p=0}^{n} \frac{(-)^{p}}{2^{p}} \frac{(n+p) !}{(n-p) ! p !^{2}} L_{p}^{s / a} \quad \text { (s/a for } n \text { even } / \text { odd }\right)
$$


See appendix for derivation. We note that the coefficients in the sum are almost identical to the coefficients in the sum for the expansion of $Q_{n}(\bar{\xi}) P_{n}(\bar{\eta})$ in terms of $L_{n}$ (Eq. 3.38).

The first few orders are

$$
\begin{aligned}
Q_{0}(\xi) P_{0}(\eta) & =L_{0}^{s} \\
Q_{1}(\xi) P_{1}(\eta) & =L_{0}^{a}-L_{1}^{a} \\
Q_{2}(\xi) P_{2}(\eta) & =L_{0}^{s}-3 L_{1}^{s}+\frac{3}{2} L_{2}^{s} \\
Q_{3}(\xi) P_{3}(\eta) & =L_{0}^{a}-6 L_{1}^{a}+\frac{15}{2} L_{2}^{a}-\frac{5}{2} L_{3}^{a}
\end{aligned}
$$




\subsection{Intensity plots}

We present intensity plots of the irregular spheroidal harmonics and logopoles on the $x z$-plane. The spheroidal harmonic singularities range from $z=-2$ to 2 , while the logopole singularities range from $z=0$ to 2 .

The colour-bars give the value of the function - note that since $Q_{0} P_{0}$ is non-negative, the dark blue corresponds to a value of zero, but for higher orders, zero is green.
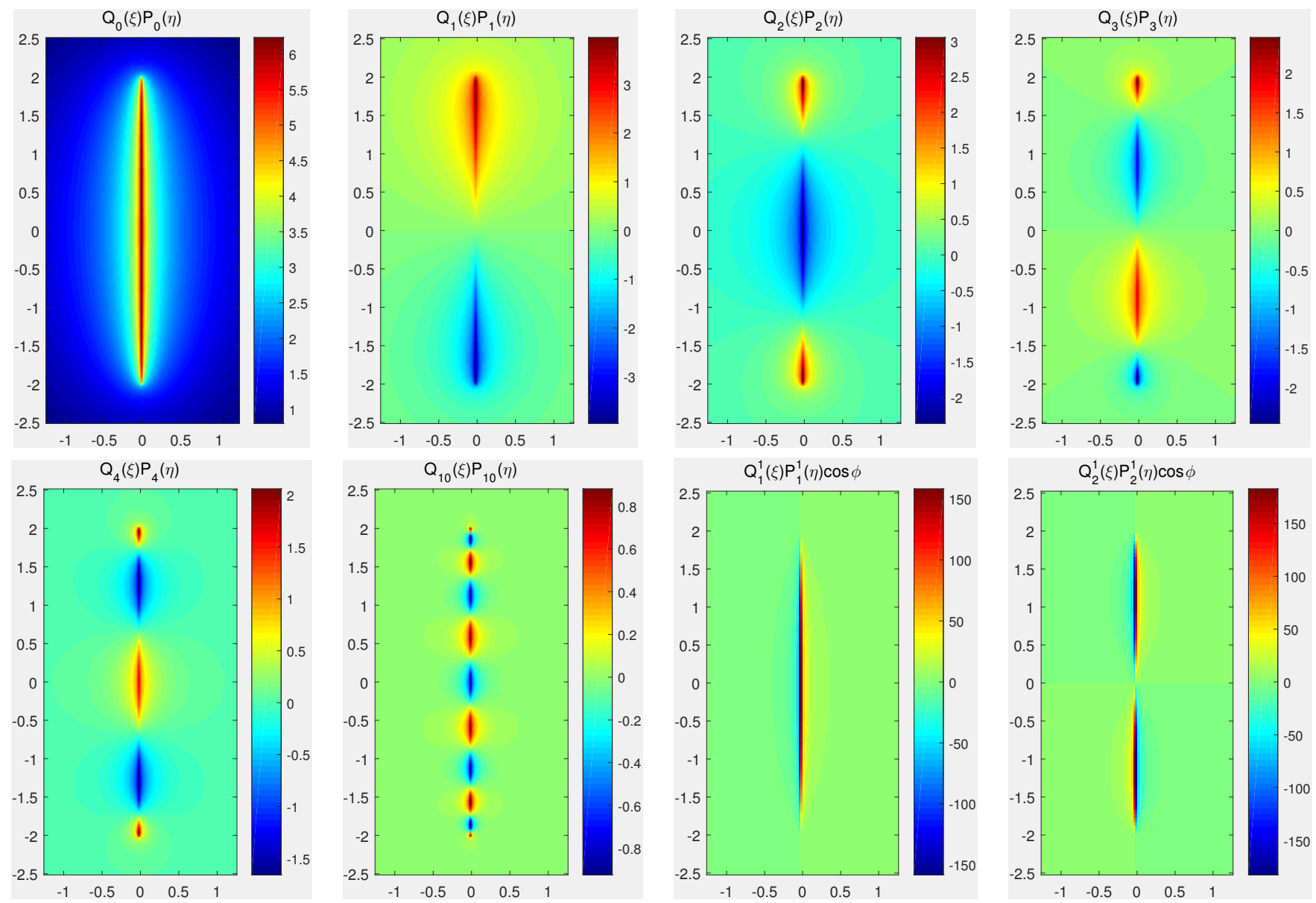

Figure 3.1: intensity plots of irregular spheroidal harmonics for $m=0$ and 1 . The horizontal axis of the graph represents $x$ in $3 \mathrm{D}$ space, and the vertical axis represents $z$. 
For the logopole plots, all logopoles are actually infinite along the central line from $z=0$ to 2 , although it is not clearly seen with the higher orders because they do not diverge until very close to the singularity, so they appear smooth where the plot is interpolated across $x=0$ (they cannot be evaluated at $x=0$ ). The logopoles were calculated by recurrence and checked with the expansion in terms of barred spheroidal harmonics. The equipotentials of the logopoles approach spheres as $r \rightarrow \infty$. They are positive everywhere in space (since they are proportional to the potential created by a positive charge distribution). Note $L_{0}$ and $Q_{0}(\xi) P_{0}(\eta)$ are the same functions but translated and scaled.
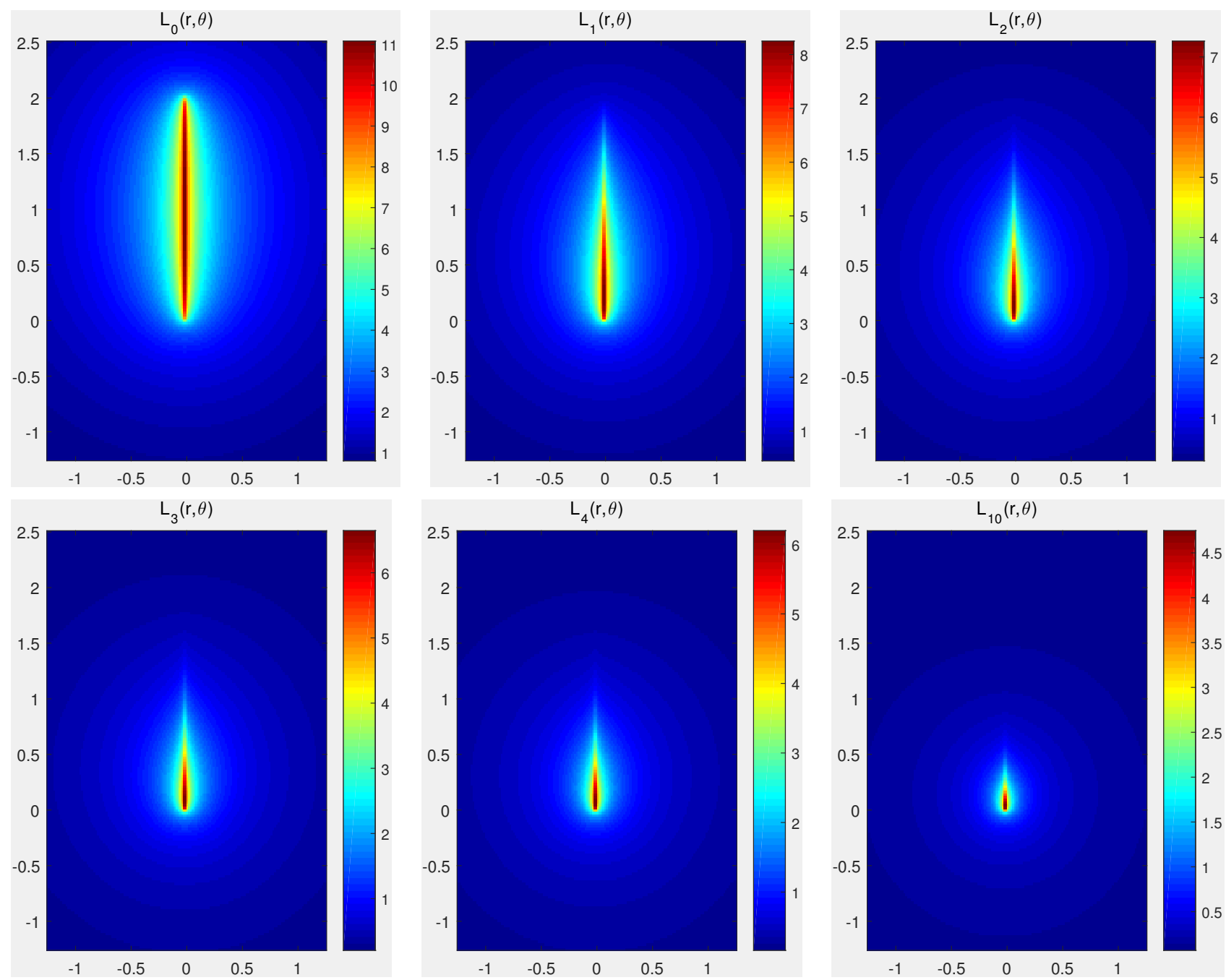

Figure 3.2: intensity plots of logopoles. The horizontal axis of the graph represents $x$ in $3 \mathrm{D}$ space, and the vertical axis represents $z$. 


\section{Chapter 4}

\section{Applications - Point source interaction with sphere}

Problems involving a point source interacting with a dielectric sphere were the main motivation for this project, in particular to find a faster converging solution to the electrostatic approximation of the decay rate of an oscillating dipole near a dielectric sphere. We hoped to generalise this to the Helmholtz equation to be able to apply these calculations to the exact decay rate, but are yet unsuccessful (see conclusions and outlook). These problems have motivated investigating logopoles and offset spheroidal harmonics since both were useful as solutions.

\subsection{General definitions}

This chapter deals with a sphere radius $a$ centred at the origin $\mathrm{O}$ (see figure 4.1), and separately with three electrostatic point sources - a charge, a dipole oriented perpendicular to the surface and a dipole parallel to the surface. The permittivity of the surroundings is $\epsilon_{1}$ and the relative dielectric constant of the sphere to the medium is $\epsilon$. The point source is located at $\mathrm{P}$ where $(x, y, z)=\left(0,0, R_{P}\right)$. Image point sources appear at $\mathrm{I}=\left(0,0, R_{I}\right)$ where $R_{I}=a^{2} / R_{P}$. Coordinates with subscript $P$ are centred at point $\mathrm{P}$, and similarly for point I. For each of the three cases we consider both $R_{P}>a$ and $R_{P}<a$, and

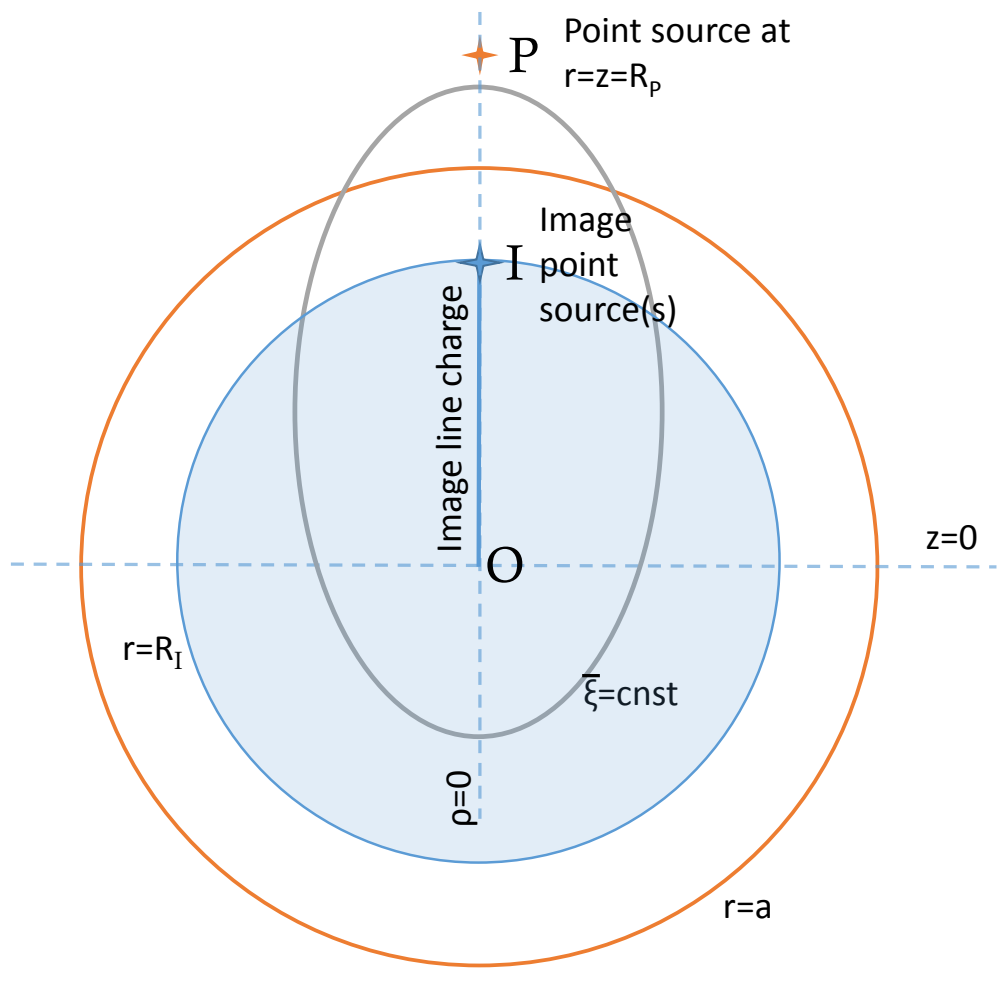
calculate the potential inside and out.

Figure 4.1: schematic representation of the problem. The spheroid at constant $\bar{\xi}$ is not real but we will use these offset "barred" spheroidal coordinates in a solution of this problem.

$$
\begin{aligned}
& z_{P}=z-R_{P}, \quad r_{P}=\sqrt{\rho^{2}+\left(z-R_{P}\right)^{2}}, \quad \cos \theta_{P}=\frac{z_{P}}{r_{P}} \\
& z_{I}=z-R_{I}, \quad r_{I}=\sqrt{\rho^{2}+\left(z-R_{I}\right)^{2}}, \quad \cos \theta_{I}=\frac{z_{I}}{r_{I}}
\end{aligned}
$$




\subsection{Point charge near sphere}

\subsubsection{Standard solutions using spherical harmonics}

A point charge $q$ is at point $\mathrm{P}$ above the sphere. To derive the potential outside the sphere, it is useful to expand the incident potential $V_{\text {inc }}^{\text {ch }}$ created by the charge in terms of regular solid spherical harmonics centred at O, using Eq. 2.11 for $n=0$ :

$$
V_{i n c}^{c h}=V_{0}^{c h} \frac{a}{r_{P}}=V_{0}^{c h} \frac{a}{R_{P}} \sum_{n=0}^{\infty}\left(\frac{r}{R_{P}}\right)^{n} P_{n}(\cos \theta) \quad\left(r<R_{P}\right)
$$

where

$$
V_{0}^{c h}=\frac{q}{4 \pi \epsilon_{0} \epsilon_{1} a}
$$

Call potential inside the sphere $V_{i n}^{c h}$, and outside $V_{\text {out }}^{c h}=V_{i n c}^{c h}+V_{r e f}^{c h}$. The reflected potential $V_{r e f}^{c h}(\mathbf{r})$ can be derived by assuming solid harmonic expansions of the potential inside and out and applying electrostatic boundary conditions:

$$
\begin{aligned}
V_{\text {in }}^{\text {ch }} & =\sum_{n=0}^{\infty} a_{n} r^{n} P_{n}(\cos \theta) & V_{\text {ref }}^{\text {ch }}=\sum_{n=0}^{\infty} b_{n} r^{-n-1} P_{n}(\cos \theta) \\
V_{\text {out }}^{\text {ch }} & =V_{\text {in }}^{\text {ch }} ; & \epsilon \partial_{r} V_{\text {in }}^{\text {ch }}=\partial_{r} V_{\text {out }}^{\text {ch }} \text { at } r=a
\end{aligned}
$$

Applying these gives simultaneous equations to solve for $a_{n}$ and $b_{n}$. The reflected potential is

$$
V_{\text {ref }}^{c h}=-V_{0}^{c h}(\epsilon-1) \sum_{n=0}^{\infty} \frac{n}{n(\epsilon+1)+1}\left(\frac{R_{I}}{r}\right)^{n+1} P_{n}(\cos \theta)
$$

This solution is slowly convergent when the source and the evaluation point are near the surface (often the most interesting case). We now reformulate Eq. 4.4 to increase the rate of convergence, as done by [9].

\subsubsection{Separation of image sources}

The convergence of Eq. 4.4 can be improved by separating out an image charge from the solution. To do this, split the fraction $n /[n(\epsilon+1)+1]$ to isolate the dominant term as $n \rightarrow \infty$ :

$$
\frac{n}{n(\epsilon+1)+1}=\frac{1}{\epsilon+1}-\frac{1}{(\epsilon+1)[n(\epsilon+1)+1]}
$$

The sum over the first fraction can be recognised as the potential due to a point charge located at I, since

$$
\sum_{n=0}^{\infty}\left(\frac{R_{I}}{r}\right)^{n+1} P_{n}(\cos \theta)=\frac{R_{I}}{r_{I}} .
$$

This identity can be seen as a translation relation: Eq. 2.10 for $n=0$. Then we have for the potential:

$$
V_{\text {ref }}^{c h}=-V_{0}^{c h} \frac{\epsilon-1}{\epsilon+1}\left[\frac{R_{I}}{r_{I}}-\sum_{n=0}^{\infty} \frac{1}{n(\epsilon+1)+1}\left(\frac{R_{I}}{r}\right)^{n+1} P_{n}(\cos \theta)\right]
$$

This solution converges faster but is still slow. We can continue to isolate image terms to improve convergence; by separating the next dominant term in the fraction:

$$
\frac{1}{n(\epsilon+1)+1}=\frac{1}{(\epsilon+1)(n+1)}+\frac{\epsilon}{(\epsilon+1)(n+1)[n(\epsilon+1)+1]}
$$


Although the separation of $1 /(n+1)$ seems arbitrary (as opposed to $1 / n$ ), it makes the calculation of the next image source easier. We now derive the analytic expression for the sum over the dominant term in Eq. 4.8. The aim is to calculate:

$$
\sum_{n=0}^{\infty}\left(\frac{R_{I}}{r}\right)^{n+1} \frac{P_{n}(\cos \theta)}{n+1}
$$

To do this, start from the generating function:

$$
\frac{1}{\sqrt{1-2 x t+t^{2}}}=\sum_{n=0}^{\infty} t^{n} P_{n}(x), \quad(|t|<1) .
$$

Integrating with respect to $\mathrm{t}$ (from 0 to $t$ ):

$$
\ln \left|\frac{t-x+\sqrt{1-2 x t+t^{2}}}{1-x}\right|=\sum_{n=0}^{\infty} \frac{t^{n+1}}{n+1} P_{n}(x), \quad(|t|<1) .
$$

Setting $x=\cos \theta$ and $t=R_{I} / r$, we obtain after simplifications:

$$
\sum_{n=0}^{\infty}\left(\frac{R_{I}}{r}\right)^{n+1} \frac{P_{n}(\cos \theta)}{n+1}=\ln \frac{r_{I}-z_{I}}{r-z} \quad\left(r>R_{I}\right)
$$

Then the potential becomes

$$
V_{r e f}^{c h}=-V_{0}^{c h} \frac{\epsilon-1}{\epsilon+1}\left[\frac{R_{I}}{r_{I}}-\frac{1}{\epsilon+1} \ln \frac{r_{I}-z_{I}}{r-z}-\frac{\epsilon}{\epsilon+1} \sum_{n=0}^{\infty} \frac{1}{(n+1)[n(\epsilon+1)+1]}\left(\frac{R_{I}}{r}\right)^{n+1} P_{n}(\cos \theta)\right] .
$$

The convergence has been improved again, but is still not ideal.

Note that the logarithmic term is singular on the line segment OI. It is interesting that this term is actually the prolate spheroidal harmonic for $n=0$ :

$$
Q_{0}(\bar{\xi}) P_{0}(\bar{\eta})=\frac{1}{2} \ln \frac{\bar{\xi}+1}{\bar{\xi}-1}=\frac{1}{2} \ln \frac{r_{I}-z_{I}}{r-z}
$$

With $\bar{\xi}=\left(r+r_{I}\right) / R, \bar{\eta}=\left(r-r_{I}\right) / R$. To derive the last equality invert the definitions of $\bar{\xi}$ and $\bar{\eta}$ :

$$
r=\frac{R}{2}(\bar{\xi}+\bar{\eta}) ; \quad r_{I}=\frac{R}{2}(\bar{\xi}-\bar{\eta}) ; \quad z=\frac{R}{2}(\bar{\xi} \bar{\eta}+1) ; \quad z_{I}=\frac{R}{2}(\bar{\xi} \bar{\eta}-1)
$$

and insert these into the r.h.s. of Eq. 4.14:

$$
\frac{r_{I}-z_{I}}{r-z}=\frac{(\bar{\xi}-\bar{\eta})-(\bar{\xi} \bar{\eta}-1)}{(\bar{\xi}+\bar{\eta})-(\bar{\xi} \bar{\eta}+1)}=\frac{(\bar{\xi}+1)(\bar{\eta}-1)}{(\bar{\xi}-1)(\bar{\eta}-1)}
$$

\subsubsection{New solution using spheroidal harmonics}

In fact, the problem of a point charge near a sphere has been solved using image theory where the entire image source was comprised of a point charge at I and a line charge on OI [6]. This provides motivation to look for an expansion of the potential in terms of irregular barred spheroidal harmonics, since they have this same singularity on the line OI. Starting from the potential with just the point image separated out (Eq. 4.7), we apply Eq. 2.28 - the expansion of irregular spherical harmonics in terms of irregular barred spheroidal harmonics (with $m=0$ ):

$$
\begin{aligned}
V_{r e f}^{c h} & =-V_{0}^{c h} \frac{\epsilon-1}{\epsilon+1}\left[\frac{R_{I}}{r_{I}}-\sum_{n=0}^{\infty} \frac{1}{n(\epsilon+1)+1}\left(\frac{R_{I}}{r}\right)^{n+1} P_{n}(\cos \theta)\right] \\
& =-V_{0}^{c h} \frac{\epsilon-1}{\epsilon+1}\left[\frac{R_{I}}{r_{I}}-\sum_{n=0}^{\infty} \frac{1}{n(\epsilon+1)+1} \frac{2}{n !^{2}} \sum_{k=n}^{\infty}(-)^{n+k}(2 k+1) \frac{(k+n) !}{(k-n) !} Q_{k}(\bar{\xi}) P_{k}(\bar{\eta})\right]
\end{aligned}
$$


Now rearrange the order of summation and relabel $n \leftrightarrow k$ to obtain:

$$
V_{\text {ref }}^{c h}=-V_{0}^{c h} \frac{\epsilon-1}{\epsilon+1}\left[\frac{R_{I}}{r_{I}}-2 \sum_{n=0}^{\infty}(2 n+1) \sum_{k=0}^{n} \frac{(-)^{n+k}}{k(\epsilon+1)+1} \frac{(n+k) !}{k !^{2}(n-k) !} Q_{n}(\bar{\xi}) P_{n}(\bar{\eta})\right]
$$

However, the sum over $k$ is numerically unstable to calculate - noticeable errors start occurring at $n \approx 20$ as seen in figure 4.2. This numerical problem can be solved by finding an alternate form for this expression. Defining

$$
\mu \equiv \frac{1}{\epsilon+1},
$$

we have

$$
\sum_{k=0}^{n} \frac{(-)^{n+k}}{k(\epsilon+1)+1} \frac{(n+k) !}{k !^{2}(n-k) !}=\mu \sum_{k=0}^{n} \frac{(-)^{n+k}}{k+\mu} \frac{(n+k) !}{k !^{2}(n-k) !}=\prod_{k=0}^{n} \frac{\mu-k}{\mu+k}
$$

See appendix for proof . These coefficients are plotted in figure 4.2 .

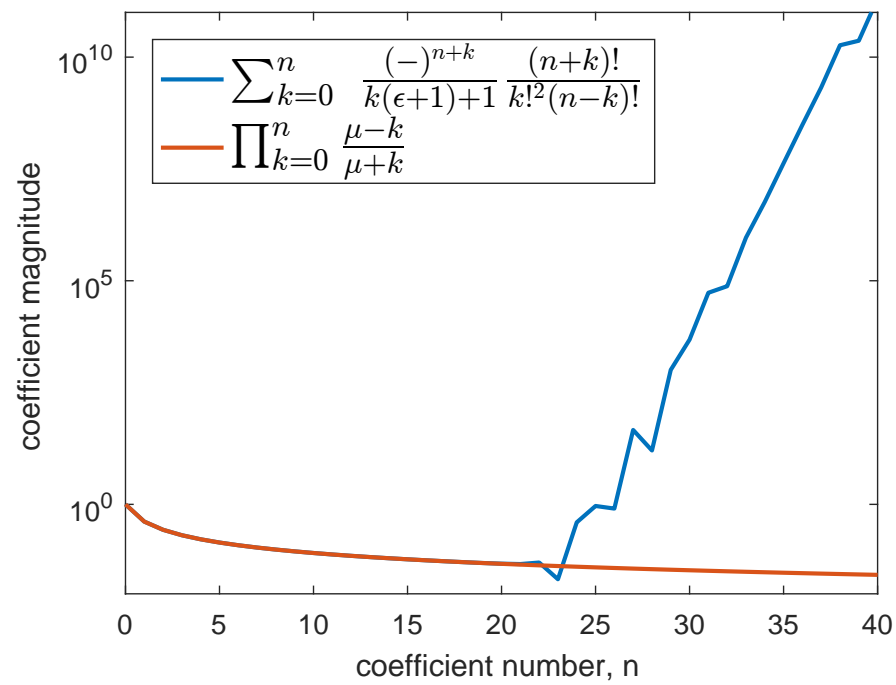

Then our final form of the potential is

$$
V_{r e f}^{c h}=-V_{0}^{c h} \frac{\epsilon-1}{\epsilon+1}\left[\frac{R_{I}}{r_{I}}-2 \sum_{n=0}^{\infty}(2 n+1) \prod_{k=0}^{n} \frac{\mu-k}{\mu+k} \quad Q_{n}(\bar{\xi}) P_{n}(\bar{\eta})\right]
$$

This converges much faster near the sphere as seen in figure 4.3. Also since the singularity of the spheroidal harmonics is only close to the surface near $\theta=0$, the series converges even faster for other areas around the surface, as opposed to the spherical harmonic expansion which converges slowly everywhere where $r \rightarrow R_{I}$. Eq. 4.21 takes only 18 terms to reach double precision accuracy at $r=R_{P}, \theta=\pi$, while 4.7 with the single point charge separated takes about 1100 terms.

The potential could instead be expanded in terms of spheroidal harmonics with foci at $z=-R_{I}$ and $R_{I}$; image sources are not unique and this problem can be solved with a longer image source. This expansion was actually derived but its convergence is slower than the barred spheroidal harmonic expansion (not shown here). It appears that an image extending from $z=0$ to $R_{I}$ is more natural. 


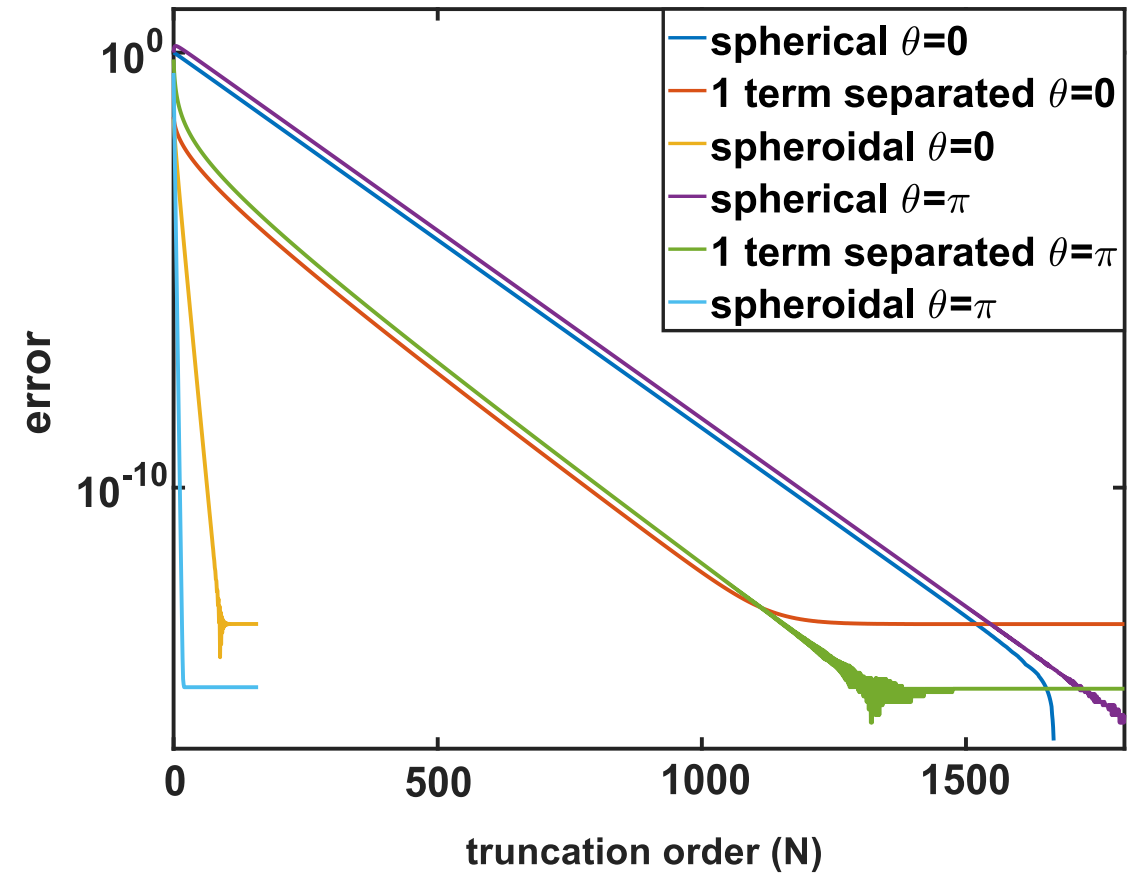

Figure 4.3: A comparison of the rates of convergence of different forms (Eqs. 4.4. 4.7. 4.21) of the reflected potential of a point charge outside a sphere. The relative error is $\left|\frac{\text { partial sum-converged sum }}{\text { converged sum }}\right|$. The distance of the charge from the origin and the radius that the potential is evaluated at are both close to the sphere $(r=$ $\left.R_{P}=1.01 a\right)$. The relative dielectric constant of the sphere and medium is $\epsilon=1.13$ but the convergence rates are almost identical for any complex $\epsilon$. The flattening out of the error at $10^{-13}-10^{-14}$ comes from the limit of double precision.

\subsubsection{Image line charge density}

As mentioned before, Lindell [6] also found an appropriate image source containing a point charge at I and a line charge on OI. Here we compare our series solution to Lindell's integral solution. They calculated the line charge density to be $q(z / R)^{-\epsilon /(\epsilon+1)}=q(z / R)^{\mu-1}$, so the potential can be written as:

$$
V_{\text {line }}=V_{0}^{\text {ch }} \frac{\epsilon-1}{(\epsilon+1)^{2}} \int_{0}^{R_{I}}\left(\frac{\tilde{z}}{R_{I}}\right)^{\mu-1} \frac{\mathrm{d} \tilde{z}}{\sqrt{\rho^{2}+(z-\tilde{z})^{2}}} .
$$

Here the tilde means a dummy variable. By comparing this solution to ours (Eq. 4.21) we can deduce the expansion of the charge distribution in terms of Legendre polynomials. We use the expression for barred spheroidal harmonics as the potential due to a line charge distribution along OI (similar to Eq. 3.53:

$$
Q_{n}(\bar{\xi}) P_{n}(\bar{\eta})=\frac{1}{2} \int_{0}^{R_{I}} \frac{P_{n}(\tilde{\bar{\eta}}) \mathrm{d} \tilde{z}}{\sqrt{\rho^{2}+(z-\tilde{z})^{2}}}
$$

Note that on the $z$-axis $\tilde{\bar{\eta}}=2 \tilde{z} / R_{I}-1$. In order for Eq. 4.22 to hold, it must be that the image line charge distribution can be expanded in terms of Legendre polynomials as follows:

$$
\left(\frac{z}{R_{I}}\right)^{\mu-1}=\left(\frac{\bar{\eta}+1}{2}\right)^{\mu-1}=\sum_{n=0}^{\infty}(2 n+1) \frac{1}{\mu} \prod_{k=0}^{n} \frac{\mu-k}{\mu+k} P_{n}(\bar{\eta})
$$

This has been checked in Matlab, but the sum converges very slowly for $\mu<<1$ (for $\mu=0.25$, successive terms actually increase up to atleast $n=10^{6}$, but may eventually converge). Insert this identity into Eq. 4.22 and use Eq. 4.23 to see that the series and integral forms of the potential are equivalent. Out of interest, Eq. 4.24 is actually a general case of Eq. 2.25 evaluated at $m=0, \bar{\xi}=1$ :

$$
\left(\frac{z}{R_{I}}\right)^{n}=\sum_{k=0}^{n} \frac{(2 k+1) n !^{2}}{(n+k+1) !(n-k) !} P_{n}(\bar{\eta})
$$

which can be seen by substituting $\mu \rightarrow n+1$ in Eq. 4.24 and relabelling summation indicies. 


\subsection{Perpendicular dipole near sphere}

We want to find a similar expression to Eq. 4.21 for the problem of a point dipole $\mathbf{p}=p \hat{\mathbf{z}}$ at $\mathrm{P}$ near the same dielectric sphere. This problem is particularly relevant for calculating the electrostatics approximation to the decay rate of an oscillating dipole near a sphere. The incident potential of the dipole is

$$
V_{i n c}^{\perp}=V_{0}^{d i p} \frac{a R_{P}}{r_{P}^{2}} \cos \theta_{P}=-V_{0}^{d i p} \frac{a}{R_{P}} \sum_{n=0}^{\infty}(n+1)\left(\frac{r}{R_{P}}\right)^{n} P_{n}(\cos \theta) \quad\left(r<R_{P}\right)
$$

where

$$
V_{0}^{d i p}=\frac{p}{4 \pi \epsilon_{0} \epsilon_{1} a R_{P}} .
$$

The above expansion uses Eq. 2.11 for $n=1$. The outside potential $V_{\text {out }}^{\perp}=V_{\text {inc }}^{\perp}+V_{\text {ref }}^{\perp}$ can be derived by assuming solid harmonic expansions of the potential inside and out of the sphere and applying electrostatic boundary conditions. The reflected potential is

$$
V_{\text {ref }}^{\perp}=V_{0}^{d i p}(\epsilon-1) \sum_{n=0}^{\infty} \frac{n(n+1)}{n(\epsilon+1)+1}\left(\frac{R_{I}}{r}\right)^{n+1} P_{n}(\cos \theta)
$$

Like with the point charge, this converges slowly for $r \rightarrow a, R_{P} \rightarrow a$.

The separation of image terms is similar to the point charge case except that there is an additional image dipole. As before, separate the leading order from the fraction:

$$
\frac{n(n+1)}{n(\epsilon+1)+1}=\frac{n}{\epsilon+1}+\frac{\epsilon n}{(\epsilon+1)[n(\epsilon+1)+1]} .
$$

The sum over the first term can be evaluated analytically using Eq. 2.10:

$$
\sum_{n=0}^{\infty} n\left(\frac{R_{I}}{r}\right)^{n+1} P_{n}(\cos \theta)=\frac{R_{I}^{2}}{r_{I}^{2}} \cos \theta_{I}
$$

which is proportional to the potential of a dipole located at I pointing along $z$. The next image term is separated out in the same manner as with the problem of the point charge outside the sphere, and again we do not separate any line source images because we want to express the line source as a series of spheroidal harmonics. We have

$$
V_{\text {ref }}^{\perp}=V_{0}^{d i p} \frac{\epsilon-1}{\epsilon+1}\left[\frac{R_{I}^{2}}{r_{I}^{2}} \cos \theta_{I}+\frac{\epsilon}{\epsilon+1} \frac{R_{I}}{r_{I}}-\frac{\epsilon}{\epsilon+1} \sum_{n=0}^{\infty} \frac{1}{n(\epsilon+1)+1}\left(\frac{R_{I}}{r}\right)^{n+1} P_{n}(\cos \theta)\right] .
$$

Inserting Eq. 2.28.

$$
V_{\text {ref }}^{\perp}=V_{0}^{d i p} \frac{\epsilon(\epsilon-1)}{(\epsilon+1)^{2}}\left[\frac{\epsilon+1}{\epsilon} \frac{R_{I}^{2}}{r_{I}^{2}} \cos \theta_{I}+\frac{R_{I}}{r_{I}}-2 \sum_{n=0}^{\infty}(2 n+1) \prod_{k=0}^{n} \frac{\mu-k}{\mu+k} Q_{n}(\bar{\xi}) P_{n}(\bar{\eta})\right]
$$

The comparison of the convergence of this expansion with the spherical harmonic expansion is similar to that of the point charge case. 


\subsection{Parallel dipole near sphere}

We find a similar expression to Eq. 4.31 for the case of a point dipole aligned parallel to the surface with $\mathbf{p}=p \hat{\mathbf{x}}$ at $\mathrm{P}$. If we know the potential due to a parallel and a perpendicular dipole then we solve for for any orientation, since any orientation of dipole can be vector decomposed into a linear combination of three orthogonal dipoles (one along each of $x, y$ and $z$ ), and because of symmetry the parallel dipole can represent either $x$ or $y$ orientations. The incident field is

$$
V_{i n c}^{\|}=V_{0}^{d i p} \frac{a R_{P} x}{r_{P}^{3}}=V_{0}^{d i p} \frac{a R_{P}}{r_{P}^{2}} P_{1}^{1}\left(\cos \theta_{P}\right) \cos \phi=V_{0}^{d i p} \frac{a}{R_{P}} \sum_{n=1}^{\infty}\left(\frac{r}{R_{P}}\right)^{n} P_{n}^{1}(\cos \theta) \cos \phi \quad\left(r<R_{P}\right)
$$

This expansion can be derived by expressing the Legendre functions $P_{n}^{1}$ in terms of the simpler Legendre polynomials:

$$
P_{n}^{1}(\cos \theta)=\frac{-n}{\sin \theta}\left[\cos \theta P_{n}(\cos \theta)-P_{n-1}(\cos \theta)\right]
$$

And using the translation relation Eq. 2.11.

By applying boundary conditions, it is straightforward to determine the potential outside the sphere $V_{\text {out }}^{\|}=V_{\text {inc }}^{\|}+V_{r e f}^{\|}$, with the reflected potential

$$
V_{r e f}^{\|}=-V_{0}^{d i p}(\epsilon-1) \cos \phi \sum_{n=1}^{\infty} \frac{n}{n(\epsilon+1)+1}\left(\frac{R_{I}}{r}\right)^{n+1} P_{n}^{1}(\cos \theta) \quad\left(r>R_{I}\right)
$$

Like with the perpendicular dipole, the fraction can be split and image terms can be separated out to make the convergence faster. Splitting the fraction as before:

$$
\frac{n}{n(\epsilon+1)+1}=\frac{1}{\epsilon+1}-\frac{1}{n(\epsilon+1)^{2}}+\frac{1}{n(\epsilon+1)^{2}(n \epsilon+n+1)}
$$

The first term results in the series

$$
\sum_{n=1}^{\infty}\left(\frac{R_{I}}{r}\right)^{n+1} P_{n}^{1}(\cos \theta) \cos \phi=R_{I}^{2} \frac{x}{r_{I}^{3}}
$$

Which is the potential of a dipole located at I, which is actually oppositely aligned to the source dipole. This identity can be shown by expressing in terms of the simpler Legendre polynomials, and using the translation relation Eq. 2.10.

The second term gives the series:

$$
\sum_{n=1}^{\infty} \frac{1}{n}\left(\frac{R_{I}}{r}\right)^{n+1} P_{n}^{1}(\cos \theta) \cos \phi
$$

In contrast with the case of a perpendicular dipole where an image point charge was identified, it is not straightforward here to recognize an analytic expression. To manipulate Eq. 4.37, we use Eq. 4.33 .

$$
\begin{aligned}
\sum_{n=1}^{\infty} \frac{1}{n}\left(\frac{R_{I}}{r}\right)^{n+1} P_{n}^{1}(\cos \theta) \cos \phi & =-\frac{\cos \phi}{\sin \theta} \sum_{n=1}^{\infty}\left(\frac{R_{I}}{r}\right)^{n+1}\left[\cos \theta P_{n}(\cos \theta)-P_{n-1}(\cos \theta)\right] \\
& =-\frac{\cos \phi}{\sin \theta}\left[\cos \theta \sum_{n=1}^{\infty}\left(\frac{R_{I}}{r}\right)^{n+1} P_{n}(\cos \theta)-\frac{R_{I}}{r} \sum_{n=0}^{\infty}\left(\frac{R_{I}}{r}\right)^{n+1} P_{n}(\cos \theta)\right] \\
& =-\frac{\cos \phi}{\sin \theta}\left[\cos \theta\left(\frac{R_{I}}{r_{I}}-\frac{R_{I}}{r}\right)-\frac{R_{I}}{r} \frac{R_{I}}{r_{I}}\right] \quad \text { using Eq. 2.10 for } n=0 \\
& =\frac{R_{I} \cos \phi}{r \sin \theta}\left(\cos \theta-\cos \theta_{I}\right), \quad \text { since } \cos \theta_{I}=\frac{r \cos \theta-R_{I}}{r_{I}} \\
& =\frac{R_{I} x}{\rho^{2}}\left(\cos \theta-\cos \theta_{I}\right)
\end{aligned}
$$


Note that the above expression is singular on the segment OI only. Because this singularity matches the singularity of the barred spheroidal harmonics, we include this term inside the spheroidal harmonic expansion. So we write the potential with only the image point dipole separated:

$$
V_{r e f}^{\|}=V_{0}^{d i p} \frac{\epsilon-1}{\epsilon+1} \cos \phi\left[-\frac{R_{I}^{2} \sin \theta_{I}}{r_{I}^{2}}+\sum_{n=1}^{\infty} \frac{1}{n(\epsilon+1)+1}\left(\frac{R_{I}}{r}\right)^{n+1} P_{n}^{1}(\cos \theta)\right]
$$

This can be converted in to barred spherical harmonics using Eq. 2.28 for $m=1$, so that the sum in Eq. 4.39 becomes

$$
\begin{aligned}
\sum_{n=1}^{\infty} \frac{1}{n(\epsilon+1)+1}\left(\frac{R_{I}}{r}\right)^{n+1} P_{n}^{1}(\cos \theta) & =\sum_{n=1}^{\infty} \frac{-2}{n(\epsilon+1)+1} \sum_{k=n}^{\infty} \frac{(-)^{n+k}}{n !(n-1) !} \frac{(k+n) !}{(k-n) !} \frac{2 k+1}{k(k+1)} Q_{k}^{1}(\bar{\xi}) P_{k}^{1}(\bar{\eta}) \\
& =-2 \sum_{n=1}^{\infty} \frac{2 n+1}{n(n+1)} \sum_{k=1}^{n} \frac{(-)^{n+k} k}{k(\epsilon+1)+1} \frac{(n+k) !}{k !^{2}(n-k) !} Q_{n}^{1}(\bar{\xi}) P_{n}^{1}(\bar{\eta})
\end{aligned}
$$

In the last step the order of summation was swapped and the indices relabelled $n \leftrightarrow k$. Now we need to convert the sum over $k$ into a product as was done with the point charge. To do this we first use the following identity which is obtained by evaluating Eq. 2.25 at $m=0, \bar{\xi}=\bar{\eta}=1$ :

$$
\sum_{k=0}^{n}(-)^{n+k} \frac{(n+k) !}{k !^{2}(n-k) !}=1
$$

Now note that

$$
\begin{aligned}
(\epsilon+1) \sum_{k=1}^{n} \frac{(-)^{n+k} k}{k(\epsilon+1)+1} \frac{(n+k) !}{k !^{2}(n-k) !}+\sum_{k=1}^{n} \frac{(-)^{n+k}}{k(\epsilon+1)+1} \frac{(n+k) !}{k !^{2}(n-k) !}=\sum_{k=1}^{n}(-)^{n+k} \frac{(n+k) !}{k !^{2}(n-k) !}=1 \\
\Rightarrow \sum_{k=1}^{n} \frac{(-)^{n+k} k}{k(\epsilon+1)+1} \frac{(n+k) !}{k !^{2}(n-k) !}=\frac{1}{\epsilon+1}\left(1-\prod_{k=1}^{n} \frac{\mu-k}{\mu+k}\right)
\end{aligned}
$$

Here we used Eq. 4.20. Then the potential is:

$$
V_{r e f}^{\|}=-V_{0}^{d i p} \frac{\epsilon-1}{\epsilon+1} \cos \phi\left[\frac{R_{I}^{2} \sin \theta_{I}}{r_{I}^{2}}-\frac{2}{\epsilon+1} \sum_{n=1}^{\infty} \frac{2 n+1}{n(n+1)}\left(\prod_{k=1}^{n} \frac{\mu-k}{\mu+k}-1\right) Q_{n}^{1}(\bar{\xi}) P_{n}^{1}(\bar{\eta})\right]
$$

Again the convergence of this series is much faster than the spherical harmonic expansion. Note the potential cannot be evaluated at $\theta=0$ because both expansions are singular; however we know by symmetry that the potential is zero along the entire $z$-axis anyway.

We only separated out one point source from the expansion because there is no point charge term for $m=1$ - instead there is a line singularity $\left(\cos \theta_{I}-\cos \theta\right) / \rho$ which corresponds to $n=0, m=1$. So the angular part $\cos \theta / \sin \theta$ is a solution to Legendre's equation for $n=0, m=1$, but it is not $Q_{0}^{1}=-1 / \sin \theta$, it is the second independent solution. As far as we know this has no name but would be analogous to $P_{0}^{1}(\cos \theta)$, although $P_{n}^{m}$ is zero for $m>n$. It turns out that the exclusion of this term from the series has only a slight difference in the resulting spheroidal harmonic expansion. Separating out this term leads to replacing the -1 that comes after the product (in Eq. 4.44) with $-(-)^{n}$. This has no effect on the rate of convergence, so we ignore it.

\subsection{Reflected electric field and dipole decay rates}

We calculate the electric field for the dipole sources because it applies to nano-optics for calculating the decay rate of an oscillating dipole near a sphere. The full decay rate must be calculated using the Helmholtz equation, but we can provide the electrostatic approximation when the wavelength is much longer than the sphere size and the Helmholtz equation reduces to Laplace's equation. 


\subsubsection{Perpendicular dipole}

In terms of spherical harmonics, the standard solution of the reflected electric field is (calculated from $\mathbf{E}=-\nabla V)$ :

$$
\mathbf{E}_{r e f}^{\perp}=E_{0}(\epsilon-1) \sum_{n=0}^{\infty} \frac{n(n+1)^{2}}{n(\epsilon+1)+1}\left(\frac{R_{I}}{r}\right)^{n+2}\left[P_{n}(\cos \theta) \hat{\mathbf{r}}+\frac{P_{n+1}(\cos \theta)-\cos \theta P_{n}(\cos \theta)}{\sin \theta} \hat{\boldsymbol{\theta}}\right]
$$

with

$$
E_{0}=\frac{p}{4 \pi \epsilon_{0} \epsilon_{1} a^{3}}
$$

Starting from the barred spheroidal harmonic expansion of the potential (Eq. 4.31, $\mathbf{E}_{r e f}^{\perp}$ can be obtained in terms of unit vectors $\hat{\mathbf{r}}$ and $\hat{\boldsymbol{\theta}}$ by using the gradient in spherical coordinates. The electric field of the point terms is easily calculated, but for the spheroidal harmonics we need the gradient in spheroidal coordinates, which we derive for the case of constant $\phi$ :

$$
\begin{aligned}
\nabla V & =-\frac{\partial}{\partial r} \hat{\mathbf{r}}-\frac{\sin \theta}{r} \frac{\partial V}{\partial \cos \theta} \hat{\boldsymbol{\theta}} \\
& =-\left(\frac{\partial \bar{\xi}}{\partial r} \frac{\partial V}{\partial \bar{\xi}}+\frac{\partial \bar{\eta}}{\partial r} \frac{\partial V}{\partial \bar{\eta}}\right) \hat{\mathbf{r}}-\left(\frac{\partial \bar{\xi}}{\partial \cos \theta} \frac{\partial V}{\partial \bar{\xi}}+\frac{\partial \bar{\eta}}{\partial \cos \theta} \frac{\partial V}{\partial \bar{\eta}}\right) \hat{\boldsymbol{\theta}} \\
& =-\frac{2}{R_{I}(\bar{\xi}-\bar{\eta})(\bar{\xi}+\bar{\eta})}\left(\left(\bar{\xi}^{2}-1\right) \frac{\partial V}{\partial \bar{\xi}}+\left(1-\bar{\eta}^{2}\right) \frac{\partial V}{\partial \bar{\eta}}\right) \hat{\mathbf{r}}-\frac{r}{r_{I}}\left(\frac{\partial V}{\partial \bar{\xi}}-\frac{\partial V}{\partial \bar{\eta}}\right) \hat{\boldsymbol{\theta}}
\end{aligned}
$$

After some algebraic manipulation, the reflected electric field is:

$$
\begin{aligned}
& \mathbf{E}_{r e f}^{\perp}=-E_{0} \frac{\epsilon(\epsilon-1)}{(\epsilon+1)^{2}}\left[\frac{\epsilon+1}{\epsilon} R_{I}^{3}\left(-2 \frac{\cos \theta}{r_{I}^{3}}+3 \frac{R_{P} r \sin ^{2} \theta}{r_{I}^{5}}\right)+\frac{R_{I}^{2}}{r_{I}^{3}}\left(R_{I} \cos \theta-r\right)\right. \\
&\left.+2 \frac{R_{I}}{r} \sum_{n=0}^{\infty}(2 n+1)(n+1) \prod_{k=1}^{n} \frac{\mu-k}{\mu+k}\left(Q_{n}(\bar{\xi}) P_{n}(\bar{\eta})+\frac{Q_{n}(\bar{\xi}) P_{n+1}(\bar{\eta})-Q_{n+1}(\bar{\xi}) P_{n}(\bar{\eta})}{\bar{\xi}-\bar{\eta}}\right)\right] \hat{\mathbf{r}} \\
&-E_{0} \frac{\epsilon(\epsilon-1)}{(\epsilon+1)^{2}} \sin \theta\left[\frac{R_{I}^{3}}{r_{I}^{3}}+\frac{\epsilon+1}{\epsilon} \frac{R_{I}^{3}}{r_{I}^{5}}\left(r^{2}+R_{P} z-2 R_{I}^{2}\right)\right. \\
&\left.-2 \frac{R_{I}}{r} \sum_{n=0}^{\infty}(2 n+1)(n+1) \prod_{k=1}^{n} \frac{\mu-k}{\mu+k}\left(\frac{Q_{n}(\bar{\xi}) P_{n}(\bar{\eta}) \cos \theta}{\sin ^{2} \theta}+\frac{r}{r_{I}}\left(\frac{Q_{n}(\bar{\xi}) P_{n+1}(\bar{\eta})}{\bar{\eta}^{2}-1}-\frac{Q_{n+1}(\bar{\xi}) P_{n}(\bar{\eta})}{\xi^{2}-1}\right)\right)\right] \hat{\boldsymbol{\theta}}
\end{aligned}
$$

Now we evaluate the field at the dipole position. The dipole position corresponds to coordinates

$$
r=R_{P}, \quad \theta=0, \quad r_{I}=r_{I P}=R_{P}-R_{I}, \quad \bar{\xi}=\bar{\xi}_{P}=2 \frac{R_{P}^{2}}{a^{2}}-1, \quad \bar{\eta}=1
$$

The self-field $E_{s f}$ is the reflected electric field at the dipole position. Note that the angular field at the dipole is zero. We present both the standard solution (Eq. 4.48) and new solution (Eq. 4.49) for comparison:

$$
\begin{aligned}
\mathbf{E}_{s f}^{\perp} & =E_{0}(\epsilon-1) \sum_{n=0}^{\infty} \frac{n(n+1)^{2}}{n(\epsilon+1)+1}\left(\frac{a^{2}}{R_{P}^{2}}\right)^{n+2} \hat{\mathbf{z}} \\
& =E_{0} \frac{\epsilon(\epsilon-1)}{(\epsilon+1)^{2}}\left\{2 \frac{\epsilon+1}{\epsilon} \frac{R_{I}^{3}}{r_{I P}^{3}}-\frac{R_{I}^{2}}{r_{I P}^{2}}+\frac{R_{I}^{2}}{R_{P} r_{I P}} \sqrt{\bar{\xi}_{P}^{2}-1} \sum_{n=0}^{\infty}(2 n+1) \prod_{k=1}^{n} \frac{\mu-k}{\mu+k} Q_{n}^{1}\left(\bar{\xi}_{P}\right)\right\} \hat{\mathbf{z}}
\end{aligned}
$$

The decay rate is deduced directly from the self-field [9]:

$$
\frac{\Gamma}{\Gamma_{0}}=1+\frac{6 \pi \epsilon_{0} \epsilon_{1}}{k_{1}^{3}} \frac{\operatorname{Im}\left(\mathbf{p}^{*} \cdot \mathbf{E}_{s f}\right)}{|\mathbf{p}|^{2}}
$$


where $\Gamma_{0}$ is the decay rate of the oscillating dipole in the absence of the sphere (which depends on the surrounding medium) and $k_{1}$ is the wavenumber in the surrounding medium. Note that $\epsilon$, the dipole moment $\mathbf{p}$ and $\mathbf{E}_{\mathrm{sf}}$ are complex in general.

$$
\frac{\Gamma_{\perp}}{\Gamma_{0}}=1+\frac{3}{2\left(k_{1} a\right)^{3}} \operatorname{Im}\left\{\frac{\epsilon(\epsilon-1)}{(\epsilon+1)^{2}}\left[2 \frac{\epsilon+1}{\epsilon} \frac{R_{I}^{3}}{r_{I P}^{3}}-\frac{R_{I}^{2}}{r_{I P}^{2}}+\frac{R_{I}^{2} \sqrt{\bar{\xi}_{P}^{2}-1}}{R_{P} r_{I P}} \sum_{n=0}^{\infty}(2 n+1) \prod_{k=1}^{n} \frac{\mu-k}{\mu+k} Q_{n}^{1}\left(\bar{\xi}_{P}\right)\right]\right\}
$$

This expression converges much faster than the corresponding expression involving spherical harmonics.

\subsubsection{Parallel dipole}

In terms of solid harmonics, the reflected electric field is

$$
\begin{aligned}
\mathbf{E}_{r e f}^{\|}=-E_{0} \sum_{n=1}^{\infty} & \frac{n(\epsilon-1)}{n(\epsilon+1)+1}\left(\frac{R_{I}}{r}\right)^{n+2}\left[(n+1) P_{n}^{1}(\cos \theta) \cos \phi \hat{\mathbf{r}}\right. \\
& \left.+\frac{n P_{n+1}^{1}(\cos \theta)-(n+1) \cos \theta P_{n}^{1}(\cos \theta)}{\sin \theta} \cos \phi \hat{\boldsymbol{\theta}}+\frac{P_{n}^{1}(\cos \theta)}{\sin \theta} \sin \phi \hat{\boldsymbol{\phi}}\right]
\end{aligned}
$$

In terms of barred spheroidal harmonics:

$$
\begin{aligned}
\mathbf{E}_{r e f}^{\|}= & -E_{0} \frac{\epsilon-1}{\epsilon+1} \cos \phi\left[\frac{R_{I}^{3} \sin \theta\left(2 r^{2}-R_{I} z-R_{I}^{2}\right)}{r_{I}^{5}}\right. \\
& \left.-\frac{2}{\epsilon+1} \frac{R_{I}}{r} \sum_{n=1}^{\infty}(2 n+1)\left(\prod_{k=1}^{n} \frac{\mu-k}{\mu+k}-1\right)\left(\frac{Q_{n}^{1}(\bar{\xi}) P_{n}^{1}(\bar{\eta})}{n}+\frac{Q_{n}^{1}(\bar{\xi}) P_{n+1}^{1}(\bar{\eta})-Q_{n+1}^{1}(\bar{\xi}) P_{n}^{1}(\bar{\eta})}{(n+1)(\bar{\xi}-\bar{\eta})}\right)\right] \hat{\mathbf{r}} \\
- & E_{0} \frac{\epsilon-1}{\epsilon+1} \cos \phi\left[R_{I}^{3}\left(\frac{\cos \theta}{r_{I}^{3}}-\frac{3 R_{I} r \sin ^{2} \theta}{r_{I}^{5}}\right)+\frac{2 R_{I}}{(\epsilon+1) \rho} \sum_{n=1}^{\infty}(2 n+1)\left(\prod_{k=1}^{n} \frac{\mu-k}{\mu+k}-1\right)\right. \\
& \left.\times\left(\frac{Q_{n}^{1}(\bar{\xi}) P_{n}^{1}(\bar{\eta})}{n} \frac{z}{r}-\frac{Q_{n}^{1}(\bar{\xi}) P_{n+1}^{1}(\bar{\eta})\left(\bar{\xi}^{2}-1\right)-Q_{n+1}^{1}(\bar{\xi}) P_{n}^{1}(\bar{\eta})\left(\bar{\eta}^{2}-1\right)}{(n+1)(\bar{\xi}+\bar{\eta})(\bar{\xi}-\bar{\eta})}\right)\right] \hat{\boldsymbol{\theta}} \\
- & E_{0} \frac{\epsilon-1}{\epsilon+1} \sin \phi\left[\frac{R_{I}^{3}}{r_{I}^{3}}+\frac{2}{\epsilon+1} \frac{R_{I}}{\rho} \sum_{n=1}^{\infty} \frac{2 n+1}{n(n+1)}\left(\prod_{k=1}^{n} \frac{\mu-k}{\mu+k}-1\right) Q_{n}^{1}(\bar{\xi}) P_{n}^{1}(\bar{\eta})\right] \hat{\boldsymbol{\phi}}
\end{aligned}
$$

To calculate the self-field $\mathbf{E}_{s f}^{\|}$(the field at the dipole position) we need to take limits as $\theta \rightarrow 0$. For the Legendre polynomials:

$$
\lim _{\theta \rightarrow 0} \frac{P_{n}^{1}(\cos \theta)}{\sin \theta}=\frac{n(n+1)}{2}
$$

Then the self-field is (note that $\hat{\boldsymbol{\theta}}=\hat{\mathbf{x}}$ at the dipole and the self field in the $z$-direction is zero):

$$
\begin{aligned}
\mathbf{E}_{s f}^{\|} & =E_{0} \frac{\epsilon-1}{2} \sum_{n=0}^{\infty} \frac{n^{2}(n+1)}{n(\epsilon+1)+1}\left(\frac{a^{2}}{R_{P}^{2}}\right)^{n+2} \hat{\mathbf{x}} \\
& =-E_{0} \frac{\epsilon-1}{\epsilon+1}\left[\frac{R_{I}^{3}}{r_{I P}^{3}}-\frac{2}{\epsilon+1} \sum_{n=1}^{\infty}(2 n+1)\left(\prod_{k=1}^{n} \frac{\mu-k}{\mu+k}-1\right) \frac{Q_{n}^{1}\left(\bar{\xi}_{P}\right)}{\sqrt{\bar{\xi}_{P}^{2}-1}}\right] \hat{\mathbf{x}}
\end{aligned}
$$

And the decay rate is

$$
\frac{\Gamma_{\|}}{\Gamma_{0}}=1-\frac{3}{2\left(k_{1} a\right)^{3}} \operatorname{Im}\left\{\frac{\epsilon-1}{\epsilon+1}\left[\frac{R_{I}^{3}}{r_{I P}^{3}}-\frac{2}{\epsilon+1} \sum_{n=1}^{\infty}(2 n+1)\left(\prod_{k=1}^{n} \frac{\mu-k}{\mu+k}-1\right) \frac{Q_{n}^{1}\left(\bar{\xi}_{P}\right)}{\sqrt{\bar{\xi}_{P}^{2}-1}}\right]\right\}
$$




\subsection{Potentials inside the sphere}

The potential inside the sphere can also be expanded in terms of (radially inverted) spheroidal harmonics. The inside potential is a series of regular spheroidal harmonics, so we would assume that this could be re-expressed in terms of regular spheroidal harmonics using Eq. 2.26, but this does not work. Instead, by changing coordinates to $\grave{r}=1 / r$ and $\grave{R}_{P}=1 / R_{P}$, the potential becomes a sum of irregular solid harmonics just like the reflected potential, and the same derivation can be applied to expand it in terms of inverted spheroidal harmonics. We present new solutions to the potentials inside the sphere for each point source. The results are similar to that of the outside potentials and the comparison of the convergence of the spheroidal harmonic solutions to the spherical harmonic solutions is very similar to figure 4.3 .

\subsubsection{Point charge}

The inside potential $V_{i n}^{\text {ch }}$ can be calculated from boundary conditions on the sphere. Note that the solution involves regular solid harmonics and the radius of convergence is $r=R_{P}$.

$$
V_{i n}^{c h}=V_{0}^{c h} \frac{a}{R_{P}} \sum_{n=0}^{\infty} \frac{2 n+1}{n(\epsilon+1)+1} \frac{r^{n}}{R_{P}^{n}} P_{n}(\cos \theta)
$$

We can separate out the image charge using the identity

$$
\frac{2 n+1}{n(\epsilon+1)+1}=\frac{2}{\epsilon+1}+\frac{\epsilon-1}{(\epsilon+1)[n(\epsilon+1)+1]}
$$

The sum over the first term on the r.h.s. can be evaluated analytically using Eq. 2.11 for $n=0$ :

$$
\sum_{n=0}^{\infty}\left(\frac{r}{R_{P}}\right)^{n} P_{n}(\cos \theta)=\frac{R_{P}}{r_{P}}
$$

which is proportional to the potential of a point charge located at $\mathrm{P}$ (the same location as the source). Inserting this into the potential:

$$
V_{i n}^{c h}=V_{0}^{c h} \frac{a}{R_{P}} \frac{1}{\epsilon+1}\left[2 \frac{R_{P}}{r_{P}}+(\epsilon-1) \sum_{n=0}^{\infty} \frac{1}{n(\epsilon+1)+1} \frac{r^{n}}{R_{P}^{n}} P_{n}(\cos \theta)\right]
$$

As demonstrated in [6], the image source can be taken to be a line singularity extending from $\mathrm{P}$ to infinity. Consider the barred spheroidal harmonics $Q_{n}(\bar{\xi}) P_{n}(\bar{\eta})$. If we radially invert coordinates about the radius $R_{P}$ - take the origin to infinity, infinity to the origin while $r=R_{P}$ stays the same, the singularity of these harmonics is now from $\mathrm{P}$ to infinity just like the image source. Therefore we define radially inverted coordinates $\grave{r}=1 / r$ and $\grave{R_{P}}=1 / R_{P}$, and rewrite the potential:

$$
V_{i n}^{c h}=V_{0}^{c h} \frac{a}{R_{P}} \frac{1}{\epsilon+1}\left[2 \frac{R_{P}}{r_{P}}+(\epsilon-1) \frac{R_{P}}{r} \sum_{n=0}^{\infty} \frac{1}{n(\epsilon+1)+1}\left(\frac{\grave{R_{P}}}{\grave{r}}\right)^{n+1} P_{n}(\cos \theta)\right]
$$

This is now a sum of irregular solid harmonics and we can apply Eq. 2.28, as long as we also transform the spheroidal coordinates:

$$
\begin{aligned}
& \grave{\xi}=\frac{\grave{r}+\grave{r}_{P}}{\grave{R}_{P}}=\frac{R_{P}}{r}+\sqrt{\frac{R_{P}^{2}}{r^{2}}-\frac{2 R_{P} \cos \theta}{r}+1} \\
& \grave{\eta}=\frac{\grave{r}-\grave{r}_{P}}{\grave{R}_{P}}=\frac{R_{P}}{r}-\sqrt{\frac{R_{P}^{2}}{r^{2}}-\frac{2 R_{P} \cos \theta}{r}+1}
\end{aligned}
$$

Then plugging Eq. 2.28 with this coordinate system into the potential, rearranging the order of summation, relabelling $n \leftrightarrow k$ and using the product form of the expansion coefficients:

$$
V_{i n}^{c h}=V_{0}^{c h} \frac{a}{R_{P}} \frac{2}{\epsilon+1}\left[\frac{R_{P}}{r_{P}}+(\epsilon-1) \frac{R_{P}}{r} \sum_{n=0}^{\infty}(2 n+1) \prod_{k=0}^{n} \frac{\mu-k}{\mu+k} Q_{n}(\grave{\xi}) P_{n}(\grave{\eta})\right]
$$


Radially inverting a solution can be used to create another solution to Laplace's equation - if $\phi(r, \theta, \phi)$ is a solution then it is straightforward to show that $r^{-1} \phi(R / r, \theta, \phi)$ is also a solution. Therefore $r^{-1} Q_{n}^{m}(\grave{\xi}) P_{n}^{m}(\grave{\eta}) e^{ \pm i m \phi}$ and $r^{-1} P_{n}^{m}(\grave{\xi}) P_{n}^{m}(\grave{\eta}) e^{ \pm i m \phi}$ are harmonics, and also $V_{i n}^{c h}$ in the form of Eq. 4.62 satisfies Laplace's equation.

\subsubsection{Perpendicular dipole}

The inside potential in terms of spheroidal harmonics is

$$
V_{i n}^{\perp}=-V_{0}^{d i p} \frac{a}{R_{P}} \sum_{n=0}^{\infty} \frac{(n+1)(2 n+1)}{n(\epsilon+1)+1} \frac{r^{n}}{R_{P}^{n}} P_{n}(\cos \theta)
$$

Before making the coordinate change, the image dipole and point charge should be separated out as with the reflected potential:

$$
V_{i n}^{\perp}=-V_{0}^{d i p} \frac{a}{R_{P}} \frac{\epsilon-1}{(\epsilon+1)^{2}}\left(\frac{R_{P}}{r_{P}}-2 \frac{\epsilon+1}{\epsilon-1} \frac{R_{P}^{2}}{r_{P}^{2}} \cos \theta_{P}+\epsilon \frac{R_{P}}{r} \sum_{n=0}^{\infty} \frac{1}{n(\epsilon+1)+1}\left(\frac{\grave{R_{P}}}{\grave{r}}\right)^{n+1} P_{n}(\cos \theta)\right)
$$

Then following the same derivation as for the inside potential for a point charge, we find:

$$
V_{i n}^{\perp}=-V_{0}^{d i p} \frac{a}{R_{P}} \frac{\epsilon-1}{(\epsilon+1)^{2}}\left[\frac{R_{P}}{r_{P}}-2 \frac{\epsilon+1}{\epsilon-1} \frac{R_{P}^{2}}{r_{P}^{2}} \cos \theta_{P}+2 \epsilon \frac{R_{P}}{r} \sum_{n=0}^{\infty}(2 n+1) \prod_{k=0}^{n} \frac{\mu-k}{\mu+k} Q_{n}(\grave{\xi}) P_{n}(\grave{\eta})\right]
$$

\subsubsection{Parallel dipole}

The inside potential in terms of spheroidal harmonics is

$$
V_{i n}^{\|}=V_{0}^{d i p} \frac{a}{R_{P}} \sum_{n=0}^{\infty} \frac{2 n+1}{n(\epsilon+1)+1} \frac{r^{n}}{R_{P}^{n}} P_{n}^{1}(\cos \theta) \cos \phi
$$

As with the reflected potential, there is only one point image source. The spheroidal harmonic expansion is:

$$
V_{i n}^{\|}=V_{0}^{d i p} \frac{a}{R_{P}} \frac{2 \cos \phi}{\epsilon+1}\left[\frac{R_{P}^{2} \sin \theta_{P}}{r_{P}^{2}}-\frac{\epsilon-1}{\epsilon+1} \frac{R_{P}}{r} \sum_{n=1}^{\infty} \frac{2 n+1}{n(n+1)}\left(\prod_{k=0}^{n} \frac{\mu-k}{\mu+k}-1\right) Q_{n}^{1}(\grave{\xi}) P_{n}^{1}(\grave{\eta})\right]
$$

\subsection{Sources inside the sphere $\left(R_{P}<a\right)$}

Results are similar when point source is inside the sphere, but not identical. The image position $\mathrm{I}=\left(0,0, R_{I}\right)$ is now outside the sphere, and the inside potential is $V_{i n}=V_{i n c}+V_{\text {ref }}$. Again the convergence of the spheroidal harmonic solutions are faster than the corresponding solutions involving spherical harmonics; the comparison in every case is very similar to that for outside sources.

\subsubsection{Point charge}

Unlike when the point charge was outside the sphere, the source potential $V_{\text {inc }}^{\text {ch }}$ should now be expanded in terms of irregular solid harmonics in order for the series to converge on the surface where boundary conditions will apply.

$$
V_{i n c}^{c h}=V_{0}^{c h} \frac{R_{P}}{r_{P}}=\sum_{n=0}^{\infty}\left(\frac{R_{P}}{r}\right)^{n+1} P_{n}(\cos \theta)
$$


Following a similar derivation to the case of the source being outside the sphere we obtain:

$$
\begin{aligned}
V_{\text {out }}^{\text {ch }} & =V_{0}^{\text {ch }} \epsilon \sum_{n=0}^{\infty} \frac{2 n+1}{n(\epsilon+1)+1}\left(\frac{R_{P}}{r}\right)^{n+1} P_{n}(\cos \theta) \\
& =V_{0}^{\text {ch }} \frac{2 \epsilon}{\epsilon+1}\left[\frac{R_{P}}{r_{P}}+(\epsilon-1) \sum_{n=0}^{\infty}(2 n+1) \prod_{k=0}^{n} \frac{\mu-k}{\mu+k} Q_{n}(\bar{\xi}) P_{n}(\bar{\eta})\right]
\end{aligned}
$$

and the reflected potential inside the sphere is:

$$
\begin{aligned}
V_{r e f}^{c h} & =V_{0}^{c h} \frac{R_{P}}{a}(\epsilon-1) \sum_{n=0}^{\infty} \frac{n+1}{n(\epsilon+1)+1}\left(\frac{r}{R_{I}}\right)^{n} P_{n}(\cos \theta) \\
& =V_{0}^{c h} \frac{a}{R_{P}} \frac{\epsilon-1}{\epsilon+1}\left[\frac{R_{P}}{r_{I}}+2 \epsilon \frac{R_{P}}{r} \sum_{n=0}^{\infty}(2 n+1) \prod_{k=0}^{n} \frac{\mu-k}{\mu+k} \quad Q_{n}(\grave{\xi}) P_{n}(\grave{\eta})\right]
\end{aligned}
$$

This time the radially inverted coordinates are inverted about $r=R_{I}$.

\subsubsection{Perpendicular dipole}

The inside and outside potentials in this case are:

$$
\begin{aligned}
V_{\text {inc }}^{\perp} & =V_{0}^{\text {dip }} \frac{a}{R_{P}} \frac{R_{P}^{2}}{r_{P}^{2}} \cos \theta_{P}=V_{0}^{\text {dip }} \sum_{n=1}^{\infty} n\left(\frac{R_{P}}{r}\right)^{n+1} P_{n}(\cos \theta) \quad\left(r<R_{P}\right) \\
V_{\text {out }}^{\perp} & =V_{0}^{\text {dip }} \frac{a}{R_{P}} \sum_{n=0}^{\infty} \frac{n(2 n+1)}{n(\epsilon+1)+1}\left(\frac{R_{P}}{r}\right)^{n+1} P_{n}(\cos \theta) \\
& =V_{0}^{\text {dip }} \frac{a}{R_{P}} \frac{\epsilon-1}{(\epsilon+1)^{2}}\left[\frac{R_{P}}{r_{P}}+2 \frac{\epsilon+1}{\epsilon-1} \frac{R_{P}^{2}}{r_{P}^{2}} \cos \theta_{P}-2 \sum_{n=0}^{\infty}(2 n+1) \prod_{k=0}^{n} \frac{\mu-k}{\mu+k} Q_{n}(\bar{\xi}) P_{n}(\bar{\eta})\right] \\
V_{\text {ref }}^{\perp} & =-V_{0}^{d i p}(\epsilon-1) \sum_{n=0}^{\infty} \frac{n^{2}}{n(\epsilon+1)+1}\left(\frac{r}{R_{I}}\right)^{n} P_{n}(\cos \theta) \\
& =V_{0}^{\text {dip }} \frac{\epsilon-1}{(\epsilon+1)^{2}}\left[(\epsilon+2) \frac{R_{I}}{r_{I}}+(\epsilon+1) \frac{R_{I}^{2}}{r_{I}^{2}} \cos \theta_{I}-2 \frac{R_{I}}{r} \sum_{n=0}^{\infty}(2 n+1) \prod_{k=0}^{n} \frac{\mu-k}{\mu+k} Q_{n}(\grave{\xi}) P_{n}(\grave{\eta})\right]
\end{aligned}
$$

\subsubsection{Parallel dipole}

The inside and outside potentials in this case are:

$$
\begin{aligned}
V_{\text {inc }}^{\|} & =V_{0}^{\text {dip }} \frac{a R_{P} x}{r_{P}^{3}}=V_{0}^{\text {dip }} \frac{a}{R_{P}} \cos \phi \sum_{n=1}^{\infty}\left(\frac{R_{P}}{r}\right)^{n+1} P_{n}^{1}(\cos \theta) \\
V_{\text {out }}^{\|} & =V_{0}^{d i p} \frac{a}{R_{P}} \epsilon \cos \phi \sum_{n=1}^{\infty} \frac{2 n+1}{n(\epsilon+1)+1}\left(\frac{R_{P}}{r}\right)^{n+1} P_{n}^{1}(\cos \theta) \\
& =V_{0}^{d i p} \frac{a}{R_{P}} \frac{2 \epsilon}{\epsilon+1} \cos \phi\left[\frac{R_{P}^{2}}{r_{P}^{2}} \sin \theta_{P}-\frac{\epsilon-1}{\epsilon+1} \sum_{n=1}^{\infty} \frac{2 n+1}{n(n+1)}\left(\prod_{k=0}^{n} \frac{\mu-k}{\mu+k}-1\right) Q_{n}^{1}(\bar{\xi}) P_{n}^{1}(\bar{\eta})\right] \\
V_{r e f}^{\|} & =V_{0}^{d i p}(\epsilon-1) \cos \phi \sum_{n=0}^{\infty} \frac{n+1}{n(\epsilon+1)+1}\left(\frac{r}{R_{I}}\right)^{n} P_{n}^{1}(\cos \theta) \\
& =V_{0}^{d i p} \frac{\epsilon-1}{\epsilon+1} \cos \phi\left[-\frac{R_{I}^{2}}{r_{I}^{2}} \sin \theta_{I}+\frac{2 \epsilon}{\epsilon+1} \frac{R_{I}}{r} \sum_{n=1}^{\infty} \frac{2 n+1}{n(n+1)}\left(\prod_{k=0}^{n} \frac{\mu-k}{\mu+k}-1\right) Q_{n}^{1}(\grave{\xi}) P_{n}^{1}(\grave{\eta})\right]
\end{aligned}
$$




\subsection{Logopole solutions}

Logopoles can be used as part of the series expansion for problems with point sources outside spherical objects. The series converge faster than the spheroidal harmonic expansions but the spheroidal harmonic solutions are simpler. The logopole solution for a perpendicular dipole near a sphere was originally found by Eric Le Ru and I used his method to find the point charge solution.

\subsubsection{Potential of point charge near sphere}

We will derive the logopole solution for the reflected potential starting from the standard solution:

$$
V_{r e f}^{c h}=-V_{0}^{c h}(\epsilon-1) \sum_{n=0}^{\infty} \frac{n}{n(\epsilon+1)+1}\left(\frac{R_{I}}{r}\right)^{n+1} P_{n}(\cos \theta)
$$

We want to expand $n /[n(\epsilon+1)+1]$ as a sum of fractions that can be related to the coefficients in the first series definition of the logopoles. We split off terms in the same way as when we applied spheroidal harmonics to this problem, but continue indefinitely. Each term that is split will reveal the next order logopole in the solution. The fraction should be split as follows:

$$
\begin{aligned}
\frac{n}{n(\epsilon+1)+1} & =\frac{n(\epsilon+1)+1-1}{(n(\epsilon+1)+1)(\epsilon+1)} \\
& =\frac{1}{\epsilon+1}-\frac{1}{(\epsilon+1)(n(\epsilon+1)+1)} \\
& =\frac{1}{\epsilon+1}-\frac{(n+1)(\epsilon+1)}{(n+1)(\epsilon+1)^{2}(n(\epsilon+1)+1)} \\
& =\frac{1}{\epsilon+1}-\frac{1}{(n+1)(\epsilon+1)^{2}}-\frac{(1(\epsilon+1)-1)}{(n+1)(\epsilon+1)^{2}(n(\epsilon+1)+1)} \\
& =\frac{1}{\epsilon+1}-\frac{1}{(n+1)(\epsilon+1)^{2}}-\frac{(n+2)(\epsilon+1)(1(\epsilon+1)-1)}{(n+2)(n+1)(\epsilon+1)^{3}(n(\epsilon+1)+1)} \\
& =\frac{1}{\epsilon+1}-\frac{1}{(n+1)(\epsilon+1)^{2}}-\frac{1(\epsilon+1)-1}{(n+2)(n+1)(\epsilon+1)^{3}}-\frac{(1(\epsilon+1)-1)(2(\epsilon+1)-1)}{(n+2)(n+1)(\epsilon+1)^{3}(n(\epsilon+1)+1)}
\end{aligned}
$$

This process can be extended to infinity (the terms approach zero as $q \rightarrow \infty$ ):

$$
\frac{n}{n(\epsilon+1)+1}=\frac{1}{\epsilon+1}-\frac{1}{(\epsilon+1)^{2}} \sum_{q=0}^{\infty} \frac{n ! q !}{(n+q+1) !} \gamma_{q} .
$$

where $\gamma_{0}=1$,

$$
\gamma_{q}=\frac{[1(\epsilon+1)-1][2(\epsilon+1)-1] \ldots[q(\epsilon+1)-1]}{q !(\epsilon+1)^{q}}=\prod_{k=1}^{q}\left(1-\frac{1}{k(\epsilon+1)}\right) \quad q \geq 1
$$

Putting Eq. 4.79 back into the the reflected potential Eq. 4.77 .

$$
V_{r e f}^{c h}=-V_{0}^{c h} \frac{\epsilon-1}{\epsilon+1} \sum_{n=0}^{\infty}\left[1-\frac{1}{\epsilon+1} \sum_{q=0}^{\infty} \frac{n ! q !}{(n+q+1) !} \gamma_{q}\right]\left(\frac{R_{I}}{r}\right)^{n+1} P_{n}(\cos \theta)
$$

swapping the order of the summation of $n$ and $q$, using the closed form expression for the image charge term $R / r_{I}$, and the first definition of the logopoles (Eq. 3.1):

$$
=-V_{0}^{c h} \frac{\epsilon-1}{\epsilon+1}\left[\frac{R_{I}}{r_{I}}-\frac{1}{\epsilon+1} \sum_{q=0}^{\infty} \gamma_{q} L_{q}\right]
$$


The logopoles used here have their singularity from $z=0$ to $z=R_{I}$. However, it turns out that the convergence of this solution is slower than the standard solution. To derive a faster converging solution, truncate the sum in Eq. 4.79 at some $Q$ and add the remainder:

$$
\frac{n}{n(\epsilon+1)+1}=\frac{1}{\epsilon+1}-\frac{1}{(\epsilon+1)^{2}}\left[\sum_{q=0}^{Q} \frac{n ! q !}{(n+q+1) !} \gamma_{q}+\frac{n !(Q+1) ! \gamma_{Q+1}}{(n+Q+1) !} \frac{\epsilon+1}{n(\epsilon+1)+1}\right]
$$

This time putting Eq. 4.82 back into the the reflected potential Eq. 4.77;

$$
\begin{aligned}
V_{r e f}^{c h} & =-V_{0}^{c h} \frac{\epsilon-1}{\epsilon+1} \sum_{n=0}^{\infty}\left[1-\frac{1}{\epsilon+1} \sum_{q=0}^{Q} \frac{n ! q !}{(n+q+1) !} \gamma_{q}-\frac{n !(Q+1) !}{(n+Q+1) !} \frac{\gamma_{Q+1}}{n(\epsilon+1)+1}\right]\left(\frac{R_{I}}{r}\right)^{n+1} P_{n}(\cos \theta) \\
& =-V_{0}^{c h} \frac{\epsilon-1}{\epsilon+1}\left[\frac{R_{I}}{r_{I}}-\frac{1}{\epsilon+1} \sum_{q=0}^{Q} \gamma_{q} L_{q}-\gamma_{Q+1} \sum_{n=0}^{\infty} \frac{n !(Q+1) !}{(n+Q+1) !} \frac{1}{n(\epsilon+1)+1}\left(\frac{R_{I}}{r}\right)^{n+1} P_{n}(\cos \theta)\right]
\end{aligned}
$$

The sum over $q$ is chosen to continue up to some $Q$, then this $Q$ must be used in the next sum over $n$, which can be summed up to say $N$. So there are two parameters that determine the accuracy of the solution. The logopole part of the solution can be seen as an approximation, and the spherical harmonic part is then the remainder. Even though the logopole approximation converges slowly, the remainder converges very fast. At the dipole position, we found that the total number of terms $N+Q$ to reach a given accuracy is minimised if $N=Q$. For $r=R_{P}=1.01 a, \theta=0$, the solution takes $N=Q=18$ terms to reach double precision $\left(\approx 10^{-15}\right)$. In comparison, the spheroidal harmonic solution takes about 100 terms, while the spherical harmonic solution (with the point charge separated) takes about 1100 terms. The rates of convergence of the logopole solutions for different $Q$ are compared in figure 4.8.1.

It is interesting to compare the image line charge distribution to that of the logopoles and spheroidal harmonics. The logopoles are the potential created by the charge distribution $(1-z / R)^{n}$ which is qualitatively similar to the the charge distribution in the problem $(z / R)^{\mu-1}$ : both are positive and decrease from the origin to the point I. The spheroidal harmonics are the potential created by the line charge distribution of a Legendre function which has positive and negative regions, but despite this the spheroidal harmonic expansion converges faster.

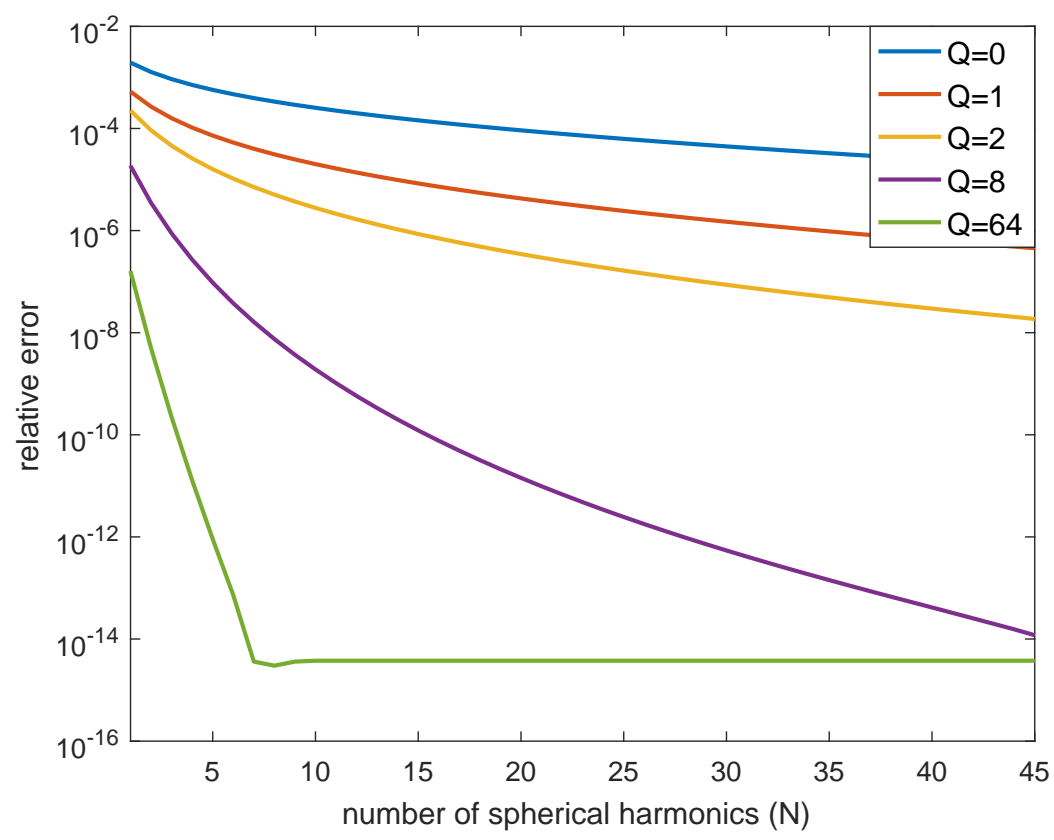

Figure 4.4: Comparisons of the rate convergence of the spherical harmonic part of the logopole solutions for different $Q$. The relative error is $\left|\frac{\text { partial sum-converged sum }}{\text { converged sum }}\right|$. For $Q>0$, the partial sum starts with the initial sum over logopoles. As $Q$ increases, the next sum over $n$ starts with a smaller magnitude and converges faster. The potential is evaluated at $r=R_{P}=$ $1.01 a, \theta=0$, and $\epsilon=1.2$ but the results are qualitatively $\epsilon$ independent. For $Q=64$ the flat base of the curve is due to limited floating point precision. 


\subsubsection{Perpendicular dipole near sphere}

The application of logopoles to this problem is similar to that of the point charge near a sphere problem. Starting from the expression with the image point sources separated:

$$
V_{\text {ref }}^{\perp}=V_{0}^{\text {dip }} \frac{\epsilon-1}{\epsilon+1}\left[\frac{R_{I}^{2}}{r_{I}^{2}} \cos \theta_{I}+\frac{\epsilon}{\epsilon+1} \frac{R_{I}}{r_{I}}-\frac{\epsilon}{\epsilon+1} \sum_{n=0}^{\infty} \frac{1}{n(\epsilon+1)+1}\left(\frac{R_{I}}{r}\right)^{n+1} P_{n}(\cos \theta)\right],
$$

Rearranging Eq. 4.82 .

$$
\frac{1}{n(\epsilon+1)+1}=\frac{1}{\epsilon+1}\left[\sum_{q=0}^{Q} \frac{n ! q !}{(n+q+1) !} \gamma_{q}+\frac{n !(Q+1) ! \gamma_{Q+1}}{(n+Q+1) !} \frac{\epsilon+1}{n(\epsilon+1)+1}\right]
$$

And inserting this into the potential:

$$
\begin{aligned}
V_{\text {ref }}^{\perp} & =V_{0}^{\operatorname{dip}} \frac{\epsilon-1}{\epsilon+1}\left[\frac{R_{I}^{2}}{r_{I}^{2}} \cos \theta_{I}+\frac{\epsilon}{\epsilon+1} \frac{R_{I}}{r_{I}}\right. \\
& \left.-\frac{\epsilon}{(\epsilon+1)^{2}}\left(\sum_{q=0}^{Q} \gamma_{q} L_{q}+\gamma_{Q+1} \sum_{n=0}^{\infty} \frac{n !(Q+1) !}{(n+Q+1) !} \frac{\epsilon+1}{n(\epsilon+1)+1}\left(\frac{R_{I}}{r}\right)^{n+1} P_{n}(\cos \theta)\right)\right]
\end{aligned}
$$

\subsubsection{Parallel dipole near sphere}

A definition of logopoles for $m=1$ would be helpful (just as the solutions with spherical and spheroidal harmonics involve the corresponding harmonics for $m=1$ ); this would be an investigation for future work. 


\section{Chapter 5}

\section{Generalization to Helmholtz}

The Helmholtz equation $\left(\nabla^{2}+k^{2}\right) \Phi(\mathbf{r})=0$ appears in applications involving electromagnetic radiation where the fields have harmonic time dependence $e^{i \omega t} . k$ is the wavenumber and $\omega$ the frequency. Here the spacial part of the electric potential satisfies the Helmholtz equation. This chapter attempts to generalize some of the concepts presented above from Laplace's equation where $k=0$.

The problem of a point dipole near a sphere in section 4 is related to the discrete dipole approximation for a spherical particle with a layer of surface material that can be modeled by a large number of dipoles. In the full electromagnetic problem, the spherical Bessel functions are used to express the electric fields of the dipoles. Numerical computation is problematic if the dipoles are close to the sphere; large numbers of Bessel functions are needed for each dipole, but the functions cannot be computed due to overflow the values exceed $10^{308}$ which is the largest number representable with double floating point precision. Therefore we look for alternative functions, similar to the spheroidal harmonics, that might provide solutions with faster convergence.

We begin by extending the expansions between spherical and spheroidal harmonics to the Helmholtz equation, and show that the Helmholtz Green's function can be expanded as a series of these new functions. Then we look at the problem of a radiating point source near a sphere. The point sources that we dealt with in the context of Laplace's equation are the long-wave limit of a radiating point source. Here the problem is more complicated because spherical Bessel functions replace the simple powers of $r$, which stops us from generalizing our method of solving this problem as we would hope.

\subsection{Generalisation of spherical-spheroidal harmonic expansions}

We define new functions that are some kind of analogue of the spheroidal harmonics for the Helmholtz equation (but are not the standard spheroidal wave functions). These have the same coefficients of expansion in terms of the spherical wave functions as do the barred spheroidal harmonics in terms of spherical harmonics. We define them from their expansions:

$$
\begin{aligned}
& \bar{F}_{n}^{m}=\frac{(-)^{m}}{2} \frac{(n+m) !}{(n-m) !} \sum_{p=n}^{\infty} \frac{(2 p+1) p !(p-m) !}{(p-n) !(n+p+1) !} h_{p}(k r) P_{p}^{m}(\cos \theta) \\
& \bar{f}_{n}^{m}=\frac{(n+m) !}{(n-m) !} \sum_{p=m}^{n} \frac{(-)^{n+p}(n+p) !}{p !(p+m) !(n-p) !} j_{p}(k r) P_{p}^{m}(\cos \theta)
\end{aligned}
$$

where $j_{p}(x)$ and $h_{p}(x)$ are the spherical Bessel and Hankel functions of the first kind. Eq. 5.1 is based on Eq. 2.27 where $\bar{F}_{n}$ is analogous to $Q_{n}^{m}(\bar{\xi}) P_{n}^{m}(\bar{\eta})$; the expansion coefficients are the same (except for the $(2 p+1)$ which is added because this factor appears in the expansion of the Helmholtz Green's function). Similarly Eq. 5.2 is based on Eq. 2.25 where $\bar{f}_{n}$ is analogous to $P_{n}^{m}(\bar{\xi}) P_{n}^{m}(\bar{\eta})$. These expansions can then be inverted just like Eqs. 2.26 and 2.28, because the inverse relationships rely on combinatorial identities (similar to the derivation of the expansion of logopoles in terms of spheroidal harmonics, Eq. 


$$
\begin{aligned}
& h_{n}(k r) P_{n}^{m}(\cos \theta)=\frac{2(-)^{n+m}}{n !(n-m) !} \sum_{p=n}^{\infty}(-)^{p} \frac{2 p+1}{2 n+1} \frac{(p+n) !}{(p-n) !} \frac{(p-m) !}{(p+m) !} \bar{F}_{p}^{m} \\
& j_{n}(k r) P_{n}^{m}(\cos \theta)=n !(n+m) ! \sum_{p=m}^{n} \frac{2 p+1}{(n-p) !(n+p+1) !} \frac{(p-m) !}{(p+m) !} \bar{f}_{p}^{m}
\end{aligned}
$$

\subsection{Alternate expansion of Green's function}

Green's function for the Helmholtz equation can be expanded in terms of $\bar{F}_{n}^{m}$ and $\bar{f}_{n}^{m}$ with the same coefficients as the expansion of the Laplace Green's function in terms of $Q_{n}^{m}(\bar{\xi}) P_{n}^{m}(\bar{\eta})$ and $P_{n}^{m}(\bar{\xi}) P_{n}^{m}(\bar{\eta})$. The Laplace Green's function was $\frac{1}{4 \pi\left|\mathbf{r}_{1}-\mathbf{r}_{2}\right|}$ for two points $\mathbf{r}_{1}$ and $\mathbf{r}_{2}$, and the Helmholtz Green's function is $\frac{e^{i k\left|\mathbf{r}_{1}-\mathbf{r}_{2}\right|}}{i k\left|\mathbf{r}_{1}-\mathbf{r}_{\mathbf{2}}\right|}$. Here we derive the expansion of the Laplace Green's function in terms of offset spheroidal harmonics, starting from the expansion in terms of spherical harmonics. Then we argue that the same works for Helmholtz. Start with Eq. 2.18 and introduce the arbitrary radius $R$ which will become the focal length of the spheroidal harmonics:

$$
\frac{1}{\left|\mathbf{r}_{\mathbf{1}}-\mathbf{r}_{\mathbf{2}}\right|}=\frac{1}{R} \sum_{k=0}^{\infty} \sum_{m=0}^{k}\left(2-\delta_{m 0}\right) \frac{(k-m) !}{(k+m) !} \cos m\left(\phi_{1}-\phi_{2}\right)\left(\frac{r_{1}}{R}\right)^{k} P_{k}^{m}\left(\cos \theta_{1}\right)\left(\frac{R}{r_{2}}\right)^{k+1} P_{k}^{m}\left(\cos \theta_{2}\right)
$$

This holds for $r_{1}<r_{2}$. Then insert the spheroidal harmonics using Eqs. 2.26 and 2.28 .

$$
\begin{aligned}
\frac{1}{\left|\mathbf{r}_{1}-\mathbf{r}_{\mathbf{2}}\right|}=\frac{1}{R} \sum_{k=0}^{\infty} \sum_{m=0}^{k} & \left(2-\delta_{m 0}\right) \frac{(k-m) !}{(k+m) !} \cos m\left(\phi_{1}-\phi_{2}\right) k !(k+m) ! \\
& \times \sum_{n=m}^{k} \frac{2 n+1}{(k-n) !(n+k+1) !} \frac{(n-m) !}{(n+m) !} P_{n}^{m}\left(\bar{\xi}_{1}\right) P_{n}^{m}\left(\bar{\eta}_{1}\right) \\
& \times \frac{2(-)^{k+m}}{k !(k-m) !} \sum_{p=k}^{\infty}(-)^{p}(2 p+1) \frac{(p+k) !}{(p-k) !} \frac{(p-m) !}{(p+m) !} Q_{p}^{m}\left(\bar{\xi}_{2}\right) P_{p}^{m}\left(\bar{\eta}_{2}\right)
\end{aligned}
$$

Now rearrange the order of summation. We want to have the summation over $k$ last, but it is currently first. This is best done by shifting one place at a time:

$$
\sum_{k=0}^{\infty} \sum_{m=0}^{k} \sum_{n=m}^{k} \sum_{p=k}^{\infty} \rightarrow \sum_{m=0}^{\infty} \sum_{k=m}^{\infty} \sum_{n=m}^{k} \sum_{p=k}^{\infty} \rightarrow \sum_{m=0}^{\infty} \sum_{n=m}^{\infty} \sum_{k=n}^{\infty} \sum_{p=k}^{\infty} \rightarrow \sum_{m=0}^{\infty} \sum_{n=m}^{\infty} \sum_{p=n}^{\infty} \sum_{k=n}^{p}
$$

Then we have

$$
\begin{aligned}
\frac{1}{\left|\mathbf{r}_{\mathbf{1}}-\mathbf{r}_{\mathbf{2}}\right|}=\frac{1}{R} \sum_{m=0}^{\infty} \sum_{n=m}^{\infty} \sum_{p=n}^{\infty} & \left(2-\delta_{m 0}\right) \cos m\left(\phi_{1}-\phi_{2}\right)(2 n+1)(2 p+1) \frac{(n-m) !}{(n+m) !} \frac{(p-m) !}{(p+m) !} \\
\times & P_{n}^{m}\left(\bar{\xi}_{1}\right) P_{n}^{m}\left(\bar{\eta}_{1}\right) Q_{p}^{m}\left(\bar{\xi}_{2}\right) P_{p}^{m}\left(\bar{\eta}_{2}\right) \sum_{k=n}^{p} \frac{(-)^{k+p}(p+k) !}{(k-n) !(k+n+1) !(p-k) !}
\end{aligned}
$$

This simplifies because of the identity

$$
\sum_{k=n}^{p} \frac{(-)^{k+p}(p+k) !}{(k-n) !(k+n+1) !(p-k) !}=\frac{\delta_{n p}}{2 p+1}
$$

Therefore we have (after inverting the summation over $n$ and $m$ )

$$
\frac{1}{\left|\mathbf{r}_{\mathbf{1}}-\mathbf{r}_{\mathbf{2}}\right|}=\sum_{n=0}^{\infty} 2 \frac{2 n+1}{R} \sum_{m=0}^{n}(-)^{m}\left(2-\delta_{m 0}\right) \frac{(n-m) !^{2}}{(n+m) !^{2}} P_{n}^{m}\left(\bar{\xi}_{1}\right) P_{n}^{m}\left(\bar{\eta}_{1}\right) Q_{n}^{m}\left(\bar{\xi}_{2}\right) P_{n}^{m}\left(\bar{\eta}_{2}\right) \cos m\left(\phi_{1}-\phi_{2}\right)
$$


This derivation also works for the Helmholtz equation because it relies solely on the expansion coefficients between the spherical and spheroidal harmonics/wave functions. So starting from the expansion of Green's function in spherical wave functions ( Jackson [17], p. 428) we can find the expansion in terms of $\bar{f}_{n}^{m}$ and $\bar{F}_{n}^{m}$ :

$$
\begin{aligned}
\frac{e^{i k\left|\mathbf{r}_{\mathbf{1}}-\mathbf{r}_{\mathbf{2}}\right|}}{i k\left|\mathbf{r}_{\mathbf{1}}-\mathbf{r}_{\mathbf{2}}\right|} & =\sum_{n=0}^{\infty} \sum_{m=0}^{n}\left(2-\delta_{m 0}\right)(2 n+1) \frac{(n-m) !}{(n+m) !} j_{n}\left(k r_{1}\right) h_{n}\left(k r_{2}\right) P_{n}^{m}\left(\cos \theta_{1}\right) P_{n}^{m}\left(\cos \theta_{2}\right) \cos m\left(\phi_{1}-\phi_{2}\right) \\
& =\sum_{n=0}^{\infty} 2(2 n+1) \sum_{m=0}^{n}(-)^{m}\left(2-\delta_{m 0}\right) \frac{(n-m) !^{2}}{(n+m) !^{2}} \bar{f}_{n}^{m}\left(\mathbf{r}_{1}\right) \bar{F}_{n}^{m}\left(\mathbf{r}_{\mathbf{2}}\right) \cos m\left(\phi_{1}-\phi_{2}\right)
\end{aligned}
$$

This result encourages us to look for analytic formulae or recurrence relations for $\bar{F}_{n}$ and $\bar{f}_{n}$.

\subsection{Expanding acoustic pressure with new functions}

We expand the potential due to a point source near a sphere in terms of $\bar{F}_{n}$. However, due to the more complex form of the radial part (spherical Bessel functions) this method does not simplify as much as we hoped.

It is easier to deal with acoustics in Helmholtz because in electromagnetism the electric field must be worked with which is a vector, and Green's function becomes a matrix. The velocity of a particle in space is determined by the gradient of the velocity potential $V(\mathbf{r})$. Consider a radiating monopole source on the $z$ axis at $z=R_{P}$ outside an acoustically soft sphere with radius $a$. The (adimensional) external potential radiated by the source is

$$
V_{\text {ext }}=\frac{e^{i k r_{P}}}{i k r_{P}}=\sum_{n=0}^{\infty}(2 n+1) h_{n}\left(k r_{>}\right) j_{n}\left(k r_{<}\right) P_{n}(\cos \theta)
$$

where $k$ is the wavenumber in the medium, $r_{P}=\sqrt{r^{2}-2 R_{P} z+R_{P}^{2}}, r_{<}=\min \left(r, R_{P}\right)$ and $r_{>}=$ $\max \left(r, R_{P}\right)$. This expansion is analogous to the expansion of the offset point charge in Laplace's equation and is actually a specific case of the expansion of the Helmholtz Green's function.

Assume that the scattered potential $V_{s c a}$ can be expanded as a series of $h_{n}(k r) P_{n}(\cos \theta)$. The boundary condition for a soft sphere is that the total potential must be zero at the boundary. From this the scattered potential is

$$
V_{\text {sca }}=-\sum_{n=0}^{\infty}(2 n+1) \frac{j_{n}(k a)}{h_{n}(k a)} h_{n}\left(k R_{P}\right) h_{n}(k r) P_{n}(\cos \theta)
$$

We now substitute in $\bar{F}_{n}$ using Eq. 5.3 . The expression for the velocity potential becomes

$$
\begin{aligned}
V_{s c a} & =-\sum_{n=0}^{\infty} \frac{j_{n}(k a)}{h_{n}(k a)} h_{n}\left(k R_{P}\right) \frac{2}{n !^{2}} \sum_{p=n}^{\infty}(-)^{n+p}(2 p+1) \frac{(p+n) !}{(p-n) !} \bar{F}_{p} \\
& =-2 \sum_{p=0}^{\infty}(2 p+1) \sum_{n=0}^{p} \frac{j_{n}(k a)}{h_{n}(k a)} h_{n}\left(k R_{P}\right) \frac{(-)^{n+p}}{n !^{2}} \frac{(p+n) !}{(p-n) !} \bar{F}_{p}
\end{aligned}
$$

Before with Laplace's equation, the $\frac{j_{n}(k a)}{h_{n}(k a)} h_{n}\left(k R_{P}\right)$ would have been powers of $R_{P}$ and $a$ which combine to give a new radius $R_{I}$. This $R_{I}$ was actually absorbed into the spheroidal harmonics (Eqs. 2.25 to 2.28 contain the arbitrary parameter $R$ which can be set to $\left.R_{I}\right)$. We could instead include $\frac{j_{p}(k a)}{h_{p}(k a)} h_{p}\left(k R_{P}\right)$ in the definition of $\bar{F}_{n}$ (call this new function $\hat{F}_{n}$ ):

$$
\hat{F}_{n}=\frac{1}{2} \sum_{p=n}^{\infty} \frac{(2 p+1) p !^{2}}{(p-n) !(n+p+1) !} \frac{j_{p}(k a)}{h_{p}(k a)} h_{p}\left(k R_{P}\right) h_{p}(k r) P_{p}(\cos \theta)
$$


If we repeat the above derivation with $\hat{F}_{n}$, we would have

$$
V_{s c a}=-2 \sum_{n=0}^{\infty}(2 n+1) \sum_{p=0}^{n}(-)^{n+p} \frac{(n+p) !}{p !^{2}(n-p) !} \hat{F}_{n}
$$

which can be simplified using the following identity obtained by evaluating Eq. 2.25 at $\bar{\xi}=\bar{\eta}=1, m=0$ :

$$
\sum_{p=0}^{n}(-)^{n+p} \frac{(n+p) !}{p !^{2}(n-p) !}=1
$$

so that

$$
V_{s c a}=-2 \sum_{n=0}^{\infty}(2 n+1) \hat{F}_{n}
$$

but this $\hat{F}_{n}$ would be more complicated and involve two extra parameters, so finding alternative expressions for $\bar{F}_{n}$ would be even more difficult. 


\section{Chapter 6}

\section{Conclusions and outlook}

To conclude we discuss some possible extensions of this work.

\subsection{Relationships for spheroidal harmonics}

The new relationships between spherical and spheroidal harmonics may be useful for deriving translation, scaling and rotation relations for spheroidal harmonics, which may then be useful for problems involving multiple spheroidal particles [8]. All three of these relations are given in [2] (for regular harmonics only). They were derived by expressing the spheroidal harmonics in terms of spherical harmonics (using the known relations), then applying translation/scaling/rotation relations for spherical harmonics, then reexpanding these into spheroidal harmonics (the formulas involve multiple sums). We could derive these formulas using our new relations between spherical and spheroidal harmonics instead, and possibly find simpler forms.

The known spherical-spheroidal harmonic relationships could also be used to find the T-matrix for a spheroid in the electrostatic approximation, by solving the problem with spheroidal harmonics then expanding these in terms of spherical harmonics. Integral forms of the matrix elements are known, but it would be ideal to find analytic formulae.

\subsection{Interacting dielectric spheres}

The application of spheroidal harmonics to the problem of a point source was successful and it may be possible to apply spheroidal harmonics to the more complicated problem of two interacting spheres.

This problem could potentially be solved using two bases of spheroidal harmonics - one for each sphere. To one sphere, the other acts as a series of multipoles which induce a reflected field from the first sphere which could be expanded on a basis of spheroidal harmonics, similar to the point source problems. In order to use this approach we would first have to determine the solution for any multipole outside a sphere using spheroidal harmonics.

\subsection{Logopoles}

Investigating logopoles has found many interesting formulae and a new expansion of spheroidal harmonics in terms of offset spherical harmonics of the second kind. We should investigate other electrostatics problems to see if they are physically useful.

It would be interesting to find a definition for logopoles with $m>0$ which would be particularly useful for the problem of a parallel dipole near a sphere. But there could be many different generalisations. One would be to simply replace $S_{n}$ with $S_{n}^{m}$ in Eq. 3.1 (this generalisation fits nicely with a few of the logopole formulae). Another would be to take the expansion in terms of $Q_{n}(\bar{\xi}) P_{n}(\bar{\eta})$ (Eq. 3.39) and generalise it using the same coefficients as in Eq. 2.26. 


\section{Appendix}

\section{A Proof of Eq. 3.68}

This derivation is similar to the one for the relationship between $Q_{n}(\bar{\xi}) P_{n}(\bar{\eta})$ and $L_{k}$ (Eq. 3.38).

We use the series expansion [2]:

$$
Q_{n}(\xi) P_{n}(\eta)=\sum_{\substack{k=n \\ n-k \text { even }}}^{\infty} \frac{k !}{(k-n) ! !(k+n+1) ! !} S_{k}=\sum_{\substack{k=0 \\ n-k \text { even }}}^{\infty} \frac{2^{n} k !\left(\frac{k+n}{2}\right) !}{\left(\frac{k-n}{2}\right) !(k+n+1) !} S_{k}
$$

Note the sum can be extended down to $k=0$ since all terms with $k<n$ are zero anyway. Without loss of generality, consider the even spheroidal harmonics ( $n$ and therefore $k$ are even). The symmetric logopoles are:

$$
L_{n}^{s}=\sum_{\substack{k=0 \\ k \text { even }}}^{\infty} \frac{k ! n !}{(k+n+1) !} S_{k}
$$

Now assume that the following expansion exists:

$$
\begin{aligned}
Q_{n}(\xi) P_{n}(\eta) & =\sum_{p=0}^{n} \zeta_{p}^{n} L_{p}^{s} \\
\Rightarrow \sum_{k \text { even }}^{\infty} \frac{2^{n} k !\left(\frac{k+n}{2}\right) !}{\left(\frac{k-n}{2}\right) !(k+n+1) !} S_{k} & =\sum_{p=0}^{n} \zeta_{p}^{n} \sum_{k \text { even }}^{\infty} \frac{k ! p !}{(k+p+1) !} S_{k}
\end{aligned}
$$

by the orthogonality of $S_{k}, \zeta_{p}^{n}$ must satisfy

$$
\begin{aligned}
2^{n} \frac{k ! \frac{k+n}{2}\left(\frac{k+n}{2}-1\right) \ldots\left(\frac{k+n}{2}-n+1\right)}{(n+k+1) !} & =\sum_{p=0}^{n} \frac{p ! k ! \zeta_{p}^{n}}{(p+k+1) !} \\
\Rightarrow 2^{n} \frac{k+n}{2}\left(\frac{k+n}{2}-1\right) \ldots\left(\frac{k+n}{2}-n+1\right) & =\sum_{p=0}^{n}\left(\begin{array}{c}
n+k+1 \\
n-p
\end{array}\right)(n-p) ! p ! \zeta_{p}^{n} \\
& =\sum_{q=0}^{n}\left(\begin{array}{c}
t \\
q
\end{array}\right) q !(n-q) ! \zeta_{n-q}^{n}
\end{aligned}
$$

Where $t=n+k+1$ and $q=n-p$. This is in the form where we can use the binomial coefficient formula Eq. 3.44. The polynomial on the left hand side is $p(t)=\frac{t-1}{2}\left(\frac{t-1}{2}-1\right) \ldots\left(\frac{t-1}{2}-n+1\right)$. We have

$$
a_{q}=q !(n-q) ! \zeta_{n-q}^{n}=2^{n} \sum_{i=0}^{q}(-)^{q+i}\left(\begin{array}{l}
q \\
i
\end{array}\right) \frac{i-1}{2}\left(\frac{i-1}{2}-1\right) \ldots\left(\frac{i-1}{2}-n+1\right)
$$

$p(i)$ is zero for $i=2 b+1$ for some $b$ in the range 1 to $2 n-1$. These $i$ values are all odd so the $(-)^{i}$ can be neglected. Then

$$
\zeta_{n-q}^{n}=\frac{(-)^{n-q} 2^{n}}{(n-q) !} \sum_{i=0}^{q} \frac{1}{i !(q-i) !} \prod_{b=0}^{n-1}\left(\frac{i-1}{2}-b\right)
$$

For the antisymmetric case ( $n$ odd), the same derivation as above can be used to find the same $\zeta_{p}^{n}$ - the only difference is that both $Q_{n}(\xi) P_{n}(\eta)$ and $L_{n}^{a}$ are sums over $k$ odd instead of even which does not affect the derivation.

Now we want to write this with positive integer binomial coefficients in order to use a known identity. Expand the product over $b$ :

$$
\prod_{b=0}^{n-1}\left(\frac{i-1}{2}-b\right)=\frac{1}{2^{n}}(i-1)(i-3) \ldots(i-2 n+1)
$$


All factors with $b \geq i / 2$ are negative - there are $n-i / 2$ of these so multiply by $(-)^{n-i / 2}$ and switch the sign of the negative factors:

$$
\begin{aligned}
\prod_{b=0}^{n-1}\left(\frac{i-1}{2}-b\right)= & \frac{(-)^{n-i / 2}}{2^{n}}(i-1)(i-3)(2 n-i-1)(2 n-i-3) \ldots 1 \\
= & \frac{(-)^{n-i / 2}}{2^{n}}(i-1) ! !(2 n-i-1) ! ! \\
& =\frac{(-)^{n-i / 2}}{2^{n}} \frac{i !}{2^{i / 2}(i / 2) !} \frac{(2 n-i) !}{2^{n-i / 2}(n-i / 2) !} \\
\Rightarrow \zeta_{n-q}^{n}= & \frac{(-)^{q}}{2^{n}(n-q) !} \sum_{i=0}^{q} \frac{(-)^{i / 2}}{(q-i) !} \frac{(2 n-i) !}{(i / 2) !(n-i / 2) !} \\
= & \frac{(-)^{q}}{2^{n}(n-q) !} \sum_{k=0}^{q / 2}(-)^{k} \frac{(2 n-2 k) !}{k !(q-2 k) !(n-k) !} \quad k \equiv i / 2 \\
= & \frac{(-)^{q}(2 n-q}{2^{n}}\left(\begin{array}{c}
q / 2 \\
n
\end{array}\right) \sum_{k=0}^{q}(-)^{k}\left(\begin{array}{c}
n \\
k
\end{array}\right)\left(\begin{array}{c}
2 n-2 j \\
2 n-q
\end{array}\right)
\end{aligned}
$$

This can be simplified using the relation ([18], pg 620):

$$
\sum_{k=0}^{q / 2}(-)^{k}\left(\begin{array}{l}
n \\
k
\end{array}\right)\left(\begin{array}{c}
2 n-2 k \\
2 n-q
\end{array}\right)=2^{q}\left(\begin{array}{l}
n \\
q
\end{array}\right)
$$

Therefore, subbing back in $p=n-q$ :

$$
\zeta_{p}^{n}=\frac{(-)^{p}}{2^{p}}\left(\begin{array}{c}
n+p \\
p
\end{array}\right)\left(\begin{array}{c}
n \\
p
\end{array}\right)=\frac{(-)^{p}}{2^{p}} \frac{(n+p) !}{(n-p) ! p !^{2}} .
$$

\section{B Proof of equation 4.20}

We want to prove that

$$
\sum_{k=0}^{n} \frac{(-)^{k}}{k+\mu} \frac{(n+k) !}{(n-k) ! k !^{2}}=\frac{(-)^{n}}{\mu} \prod_{k=0}^{n} \frac{\mu-k}{\mu+k}=\frac{(1-\mu)(2-\mu) \ldots(n-\mu)}{\mu(\mu+1)(\mu+2) \ldots(\mu+n)}
$$

First convert the left hand side into a single fraction:

$$
\begin{aligned}
& \frac{1}{\mu}-\frac{(n+1) n}{\mu+1}+\frac{(n+2)(n+1) n(n-1)}{(\mu+2) 2 !^{2}}+\frac{(n+3)(n+2)(n+1) n(n-1)(n-2)}{(\mu+3) 3 !^{2}}-\ldots+(-)^{n} \frac{2 n(2 n-1) \ldots 1}{(\mu+n) n !^{2}} \\
& =\frac{(\mu+1) \ldots(\mu+n)-(n+1) n \mu(\mu+2) \ldots(\mu+n)+\frac{1}{2 !^{2}}(n+2)(n+1) n(n-1) \mu(\mu+1)(\mu+3) \ldots(\mu+n)-\ldots}{\mu(\mu+1)(\mu+2) \ldots(\mu+n)}
\end{aligned}
$$

Now both the left and right hand sides are fractions with denominator $\mu(\mu+1)(\mu+2) \ldots(\mu+n)$, and the numerators are a polynomial degree $n$. The numerators can be shown to be equal if they are equal at $n+1$ points. Define $f(\mu)$ as the l.h.s. numerator and $g(\mu)$ as the r.h.s. numerator. Choose the $n+1$ points to be at $\mu=-q$ where $q=0,1,2, \ldots n$. For $g(\mu)$ it is easy to show that

$$
g(-q)=(1+q)(2+q) \ldots(n+q)=\frac{(n+q) !}{q !} .
$$

$f(\mu)$ is composed of a sum of terms containing $\left[(-)^{b} / b !^{2}\right] \mu(\mu+1) \ldots(\mu+b-1)(\mu+b+1) \ldots(\mu+n) \times(n+$ $b)(n+b-1) \ldots(n-b+1)$ for some $b \in\{0,1, \ldots n\}$. When we set $\mu=-q$, all the terms vanish except the one with $b=q$. This term is

$$
f(-q)=\frac{(-)^{q}}{q !^{2}}(n+q)(n+q-1) \ldots(n-q+1) \times(-q)(-q-1) \ldots(-1) \times(1)(2) \ldots(n-q)=\frac{(n+q) !}{q !}
$$

This applies to all $n+1$ values of $q$, so $f(\mu)=g(\mu)$, and Eq. 4.20 holds. 


\section{Matlab}

All important equations have been checked in Matlab, usually by computing both sides of the equation separately and computing the error. Ideally the error should be $\approx 10^{-15}$ if the equation is correct, because this is the maximum accuracy that Matlab (or any other program) can compute to using double floating point precision. However, sometimes this method can produce large errors even if the equation is correct, due to numerical instability. Below is a simple working example script that checks Eq. 2.26 for $m=0$.

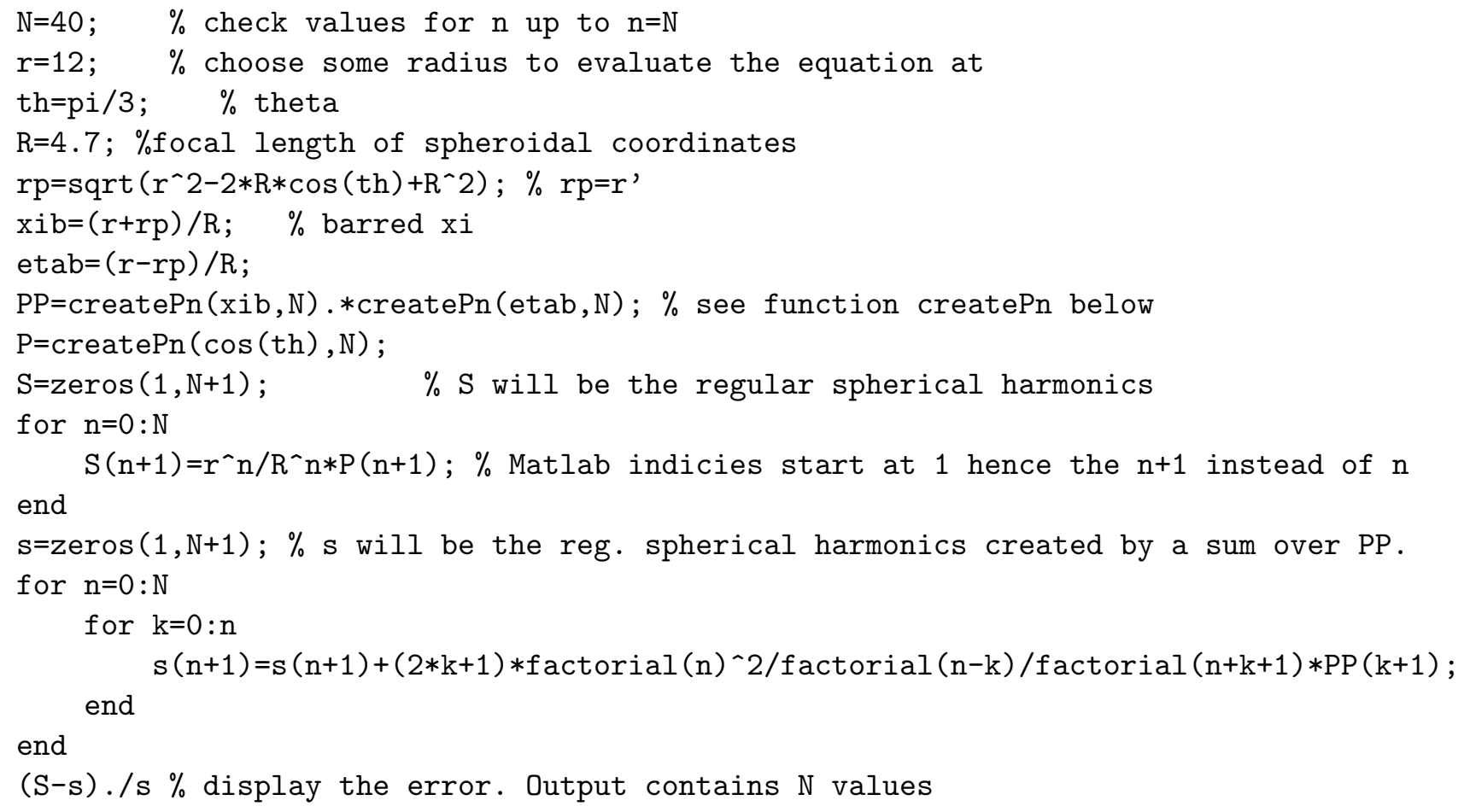

This script uses the function createPn $(x, N)$ :

function $[\mathrm{P}]=\operatorname{createPn}(\mathrm{x}, \mathrm{N})$

$\%$ create legendre functions of the second kind for a range of $x$ values

$\% \mathrm{P}$ is $\mathrm{N}$ by length $(\mathrm{x})$ and $\mathrm{P} \_\mathrm{n}$ is actually $\mathrm{P}(\mathrm{n}+1)$

$\% \mathrm{x}$ can be a vector

$\mathrm{P}=$ zeros (length $(\mathrm{x}), \mathrm{N}+1)$;

$P(:, 1)=x . / x ; \%$ a vector of ones

$\mathrm{P}(:, 2)=\mathrm{x}$;

for $n=2: N$

$\mathrm{P}(:, \mathrm{n}+1)=\left((2 * \mathrm{n}-1) * \mathrm{x} .^{\prime} \cdot * \mathrm{P}(:, \mathrm{n})-(\mathrm{n}-1) * \mathrm{P}(:, \mathrm{n}-1)\right) / \mathrm{n}$;

end

In this example only one point in space is compared, and depending on the point there may be noticeable numerical instability or slower convergence, but that is not the case with this equation. For many equations a matrix of values for $r$ and $\theta$ were checked at once.

\section{Numerical instability}

Calculating a sum or recurrence relation may produce inaccurate results due to the limited accuracy of floating point numbers. We work with double precision $(\approx 15$ digits accuracy). Consider two numbers which are identical for the first 10 digits - their subtraction can only be accurate to 5 digits. This means that sums of terms with alternating signs can be inaccurately calculated - the best way around this problem is to find an alternate form of the expression. A recurrence relation can be unstable if it involves the subtraction of similar sized terms; the best way around this is to do the recurrence backwards. 


\section{E Computation of Legendre functions}

Both $P_{n}^{m}$ and $Q_{n}^{m}$ can be computed using the recurrence relation Eq. 2.3.

$$
(n-m+1) P_{n+1}^{m}(u)=(2 n+1) u P_{n}^{m}(u)-(n+m) P_{n-1}^{m}(u)
$$

\section{E.1 Computation of $P_{n}^{m}(u)$}

$P_{n}^{m}(u)$ can be computed using Eq. 2.3 with the initial values

$$
P_{m}^{m}(u)=(2 m-1) ! !\left|1-u^{2}\right|^{m / 2}, \quad P_{m+1}^{m}(u)=(2 m+1) u P_{m}^{m}(u)
$$

This method is numerically stable - the limitation of numerical precision does not accumulate to produce large errors as $n$ increases.

\section{E.2 Computation of $Q_{n}^{m}(u)$ for $|u|>1$}

Calculating $Q_{n}^{m}$ using the recurrence relation Eq. 2.3 is numerically unstable. Computing the recursion backwards avoids this problem because it no longer involves subtracting two very similar numbers. We use the method descibed by [19, briefly the method is:

Guess a small value for $Q_{N}^{m}$ are for some large $N$, then use the modified Lentz algorithm [20] to calculate the ratio $T_{N}^{m}=Q_{N}^{m} / Q_{N-1}^{m}$ and use this to guess $Q_{N-1}^{m}$. Then recur backwards to $n=0$, where the value for $Q_{0}^{m}$ will be wrong but the ratio of all $Q_{n}^{m}$ to each other will be correct. Then rescale all $Q_{n}^{m}$ by the same factor, knowing what the correct value of $Q_{0}^{m}$ should be.

\section{Lentz algorithm for $Q_{n}^{m}$}

Here our notation is based on [20]. We want to calculate $T_{N}^{m}$ which follows the continued fraction recurrence:

$$
T_{N}^{m}=\frac{Q_{N}^{m}(u)}{Q_{N-1}^{m}(u)}=\frac{N+m}{(2 N+1) u-(N-m+1) T_{N+1}^{m}}=\frac{a_{N}^{m}}{b_{N}^{m}+T_{N+1}^{m}}
$$

The recurrence is over $N$ and we can treat each value of $m$ separately. $a_{N}^{m}$ and $b_{N}^{m}$ are:

$$
a_{N}^{m}=\frac{-N}{N-m+1} \quad b_{N}^{m}=-\frac{2 N+1}{N-m+1} u
$$

Below is the Lentz algorithm to find $T_{N}^{m}$ to a desired accuracy $\epsilon$ (ideally $\epsilon=10^{-15}$ for double precision)

$$
\begin{aligned}
& \text { set } T_{N}^{m}=\text { tiny } \\
& \text { set } C=\text { tiny } \\
& \text { set } D=0 \\
& \text { for } j=N, N+1, N+2, \ldots \text { : } \\
& \quad \text { set } D=b_{j}+a_{j} D \\
& \quad \text { if } D=0, \text { set } D=\text { tiny } \\
& \quad \text { set } D=1 / D \\
& \text { set } C=b_{j}+a_{j} / C \\
& \text { if } C=0, \text { set } C=\text { tiny } \\
& \text { set } T_{N}^{m}=T_{N}^{m} C D \\
& \quad \text { if }|C D-1|<\epsilon, \text { exit }
\end{aligned}
$$

In the notation of [20] $f=T_{N}^{m}$ and they use subscript $j$ 's $\left(f_{j}\right)$ which we can ignore as only the last $T_{N j}^{m}$ is needed. tiny should be less than $\epsilon\left|b_{j}^{m}\right|$. Here $\left|b_{j}^{m}\right|$ is always greater than $u$, so we can set tiny $=\epsilon|u|$. The last line leaves the loop when $T_{N}^{m}$ is accurate to $\epsilon$. 
Then calculate $Q_{n}^{m}$ :

Set $Q_{N}^{m}$ any number and $Q_{N-1}^{m}=Q_{N}^{m} / T_{N}^{m}$. These will be initial values for the backward recurrence on $n(\mathrm{Eq} 2.3)$. After the recurrence all these $Q_{n}^{m}$ will be off from their correct value by the same scaling factor: this can be obtained by dividing the incorrect $Q_{0}^{m}$ by the actual value of $Q_{0}^{m}$. Note that unlike $P_{n}^{m}, Q_{n}^{m}$ is non-zero for $n<m . Q_{0}^{m}$ can be obtained by using the following recurrences on $m$ :

$$
\begin{aligned}
& \sqrt{1-u^{2}} Q_{0}^{m}=(2-m) Q_{1}^{m-1}-m u Q_{0}^{m-1} \\
& \sqrt{1-u^{2}} Q_{1}^{m}=(2-m) u Q_{1}^{m-1}-m Q_{0}^{m-1}
\end{aligned}
$$

With initial values

$$
Q_{0}^{0}=\frac{1}{2} \ln \frac{u+1}{u-1}, \quad Q_{1}^{0}=u Q_{0}^{0}-1
$$

You have to compute all $Q_{0}^{m}$ and $Q_{1}^{m}$ and discard the $Q_{1}^{m}$. Multiply all $Q_{n}^{m}$ by the scaling factor for the corresponding $m$ to obtain the correct values. Note that even if $T_{N}^{m}$ is calculated incorrectly, $Q_{n}^{m}$ will be accurate for $n<<N$ which may lead to a false sense of success (see figure D.1).

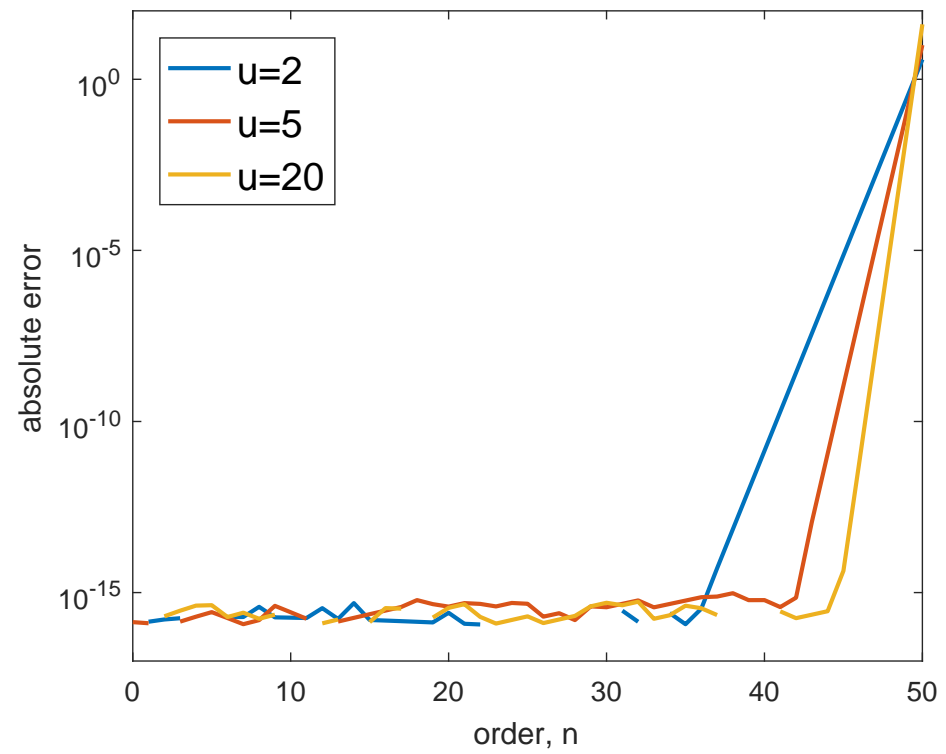

Figure D.1: the error in calculating $Q_{n}(u)$ with the incorrect ratio $T_{N}=Q_{N} / Q_{N-1}$ is plotted for various $u$. Here $N=50$. The error is the difference between the incorrect $Q_{n}$ with $T_{N}=1$ and the correct $Q_{n}$ with $T_{N}$ calculated by the Lentz algorithm $\left(T_{N} \approx 0.02-0.2\right)$. The error is quickly corrected by the backwards recurrence. 
An example Matlab script is shown below:

function [ $\mathrm{Q}]=$ createQnmbackwards $(\mathrm{x}, \mathrm{N}, \mathrm{M})$

$\%$ create Legendre functions of the second kind for a range of $\mathrm{x}$ values.

$\% \mathrm{x}$ must be a row vector with all $|\mathrm{x}(:)|>1$

$\% \mathrm{Q}$ is length( $\mathrm{x}$ ) by $\mathrm{N}$ by $M$ and $\mathrm{Q} \_\mathrm{n}^{\wedge} \mathrm{m}$ is actually $\mathrm{Q}(:, \mathrm{n}+1, \mathrm{~m}+1)$ in Matlab

$\mathrm{x}=\mathrm{x}$.'; \% convert $\mathrm{x}$ to a column vector

$\mathrm{Q}=\operatorname{zeros}($ length $(\mathrm{x}), \mathrm{N}+1, \mathrm{M}+1)$;

$\mathrm{Qs}=\operatorname{zeros}($ length $(\mathrm{x}), 2, \mathrm{M}+1) ; \quad \% \mathrm{Qs}(:, 1, \mathrm{~m}+1)$ is the scaling factor for that $\mathrm{m}$

$\%$ Need $n=0$ and $n=1$ to create all $Q s$ but in the end only need scaling factors for $n=0$

Qs $(:, 1,1)=1 / 2 * \log ((x+1) . /(x-1))$;

Qs $(:, 2,1)=x \cdot * Q s(:, 1,1)-1$;

for $m=1: M$

$\mathrm{Qs}(:, 1, \mathrm{~m}+1)=((2-\mathrm{m}) * \mathrm{Qs}(:, 2, \mathrm{~m})-\mathrm{m} * \mathrm{x} . * \mathrm{Qs}(:, 1, \mathrm{~m})) . / \operatorname{sqrt}\left(\mathrm{x} \cdot{ }^{\wedge} 2-1\right)$;

$\mathrm{Qs}(:, 2, \mathrm{~m}+1)=((2-\mathrm{m}) * \mathrm{x} . * \mathrm{Qs}(:, 2, \mathrm{~m})-\mathrm{m} * \mathrm{Qs}(:, 1, \mathrm{~m})) . / \operatorname{sqrt}\left(\mathrm{x} .{ }^{\wedge} 2-1\right)$;

end

for $m=0: M \%$ treat each $m$ separately

$\mathrm{T}=1 \mathrm{e}-14 * \mathrm{x} ; \%$ start with any small value of $\mathrm{T}=\mathrm{T}_{-} \mathrm{N}=\mathrm{Q}_{-} \mathrm{N} / \mathrm{Q}_{-}\{\mathrm{N}-1\}$

$\mathrm{C}=1 \mathrm{e}-14 * \mathrm{x} ; \%$ used to compute $\mathrm{T}$

$\mathrm{D}=0 * \mathrm{x} ; \%$ used to compute $\mathrm{T}$

exit $=0$;

$\mathrm{j}=\mathrm{N}$;

while exit $==0$

$D=-(2 * j+1) /(j-m+1) * x-(j+m) /(j-m+1) * D ;$

for $i=1:$ length $(x)$

if $D(i)==0 \quad||$ isnan(D(i)) $\%$ check if $D=0$ or undefined for any $x$ value

$D(i)=1 \mathrm{e}-14 * \mathrm{x}(i) ; \%$ reset to a small value, here epsilon $=10^{\wedge}\{-14\}$

end

end

$\mathrm{D}=1 . / \mathrm{D}$

$\mathrm{C}=-(2 * j+1) /(j-m+1) * \mathrm{x}-(j+m) /(j-m+1) . / C$;

for $i=1$ : length $(x)$

if $C(:)==0 \quad||$ isnan(C(i))

$C(i)=1 e-14 * x(i)$;

end

end

Delta $=$ C. $*$ D;

$\mathrm{T}=\mathrm{T} . *$ Delta;

if $\max (\operatorname{abs}(1-\operatorname{Delta}(:)))<1 \mathrm{e}-14$

exit=1;

end

$j=j+1$;

end

$\%$ now guess values for $Q_{-} N^{\wedge} m$ - will correct all Q_n`m later with scaling factor Qs

$\mathrm{Q}(:, \mathrm{N}+1, \mathrm{~m}+1)=1 \mathrm{e}-14 * \mathrm{x} . / \mathrm{x}$;

$\mathrm{Q}(:, \mathrm{N}, \mathrm{m}+1)=\mathrm{Q}(:, \mathrm{N}+1, \mathrm{~m}+1) . / \mathrm{T}$;

for $\mathrm{n}=\mathrm{fliplr}(2: \mathrm{N}) \quad \%$ apply backwards recurrence

$\mathrm{Q}(:, \mathrm{n}-1, \mathrm{~m}+1)=((2 * \mathrm{n}-1) * \mathrm{x} \cdot * \mathrm{Q}(:, \mathrm{n}, \mathrm{m}+1)-(\mathrm{n}-\mathrm{m}) * \mathrm{Q}(:, \mathrm{n}+1, \mathrm{~m}+1)) /(\mathrm{n}+\mathrm{m}-1) ;$

end

scale=Qs $(:, 1, m+1) . / Q(:, 1, m+1) ; \%$ all Q_n satisfy the recurrence, just need to scale them:

for $\mathrm{n}=0: \mathrm{N}$

$\mathrm{Q}(:, \mathrm{n}+1, \mathrm{~m}+1)=\mathrm{Q}(:, \mathrm{n}+1, \mathrm{~m}+1) . * \mathrm{scale} ;$

end

end 


\section{Bibliography}

[1] J. W. Perram, P. J. Stiles, Multipolar expansions of molecular fields, Proceedings of the Royal Society of London. Series A, Mathematical and PhysicalSciences, Vol. 349, No. 1656 (Apr. 13, 1976), pp. 125139

[2] G. Jansen, Transformation properties of spheroidal multipole moments and potentials J. Phys. A:Math. Gen. 33(2000) pp. 1375-1394

[3] Volodymyr I. Kushch, Micromechanics of Composites: Multipole Expansion Approach, 2013

[4] W. Thomson (Lord Kelvin), Extrait dune lettre de M. William Thomson (reported by A. M. Liouville), J. Math. Pure Appl, 10 (1845), pp. 364367.

[5] Stratton, J. A. (1941). Electromagnetic Theory. McGrawHill, New York.

[6] I.V. Lindell, Electrostatic image theory for the dielectric sphere, Radio Science Vol 27, 1992

[7] Weigan Lin, The electrostatic images of a dielectric sphere, Journal of Electrostatics 36 (1995) 129-137

[8] Wei Cai, Shaozhong Deng, Donald Jacobs, Extending the fast multipole method to charges inside or outside a dielectric sphere Journal of Computational Physics 223 (2007) 846864

[9] A. Moroz, Superconvergent Representation of the Gersten-Nitzan and Ford-Weber Nonradiative Rates, J. Phys. Chem. C 115, 19546 (2011).

[10] B Augui et al, Numerical investigation of the Rayleigh hypothesis for electromagnetic scattering by a particle J. Opt. 18 (2016) 075007

[11] William Hobson, Theory of Spherical and Ellipsoidal Harmonics, 1931

[12] Martin van Gelderen, The shift operators and translations of spherical harmonics, DEOS Progress Letter 98.1: 5767, 1998

[13] Morse and Feshbach, Methods of Theoretical Physics, 1953

[14] V. A. Antonov and A. S. Baranov, Relation between the Expansions of an External Potential in Spherical Functions and Spheroidal Harmonics, Technical Physics, Vol. 47, No. 3, 2002, pp. 361363.

[15] A. T. Benjamin, J. J. Quinn, An alternate approach to alternating sums: a method to DIE for

[16] T. H. Havelock, The wave resistance of a spheroid, Proc. R. Soc. A 131 27585, 1931

[17] J. D. Jackson, Classical Electrodynamics 3rd ed., 1998, pp 428

[18] A.P. Prudnikov, Integrals and Series, vol. 1, 1986

[19] B.I. Schneider et al. Computer Physics Communications 181 (2010) 20912097, page 2094

[20] Numerical Recipes Software, 1992, Numerical Recipies in Fortran 77: The Art of Scientific Computing, chapter 5.2 